\title{
A structural risk-neutral model for pricing and hedging power derivatives*
}

\author{
René Aïd ${ }^{\dagger}$ Luciano Campi ${ }^{\ddagger}$ Nicolas Langrené ${ }^{\S}$ \\ - Revised version, May 2011 -
}

\begin{abstract}
We develop a structural risk-neutral model for energy market modifying along several directions the approach introduced in [Aïd et al., 2009]. In particular a scarcity function is introduced to allow important deviations of the spot price from the marginal fuel price, producing price spikes. We focus on pricing and hedging electricity derivatives. The hedging instruments are forward contracts on fuels and electricity. The presence of production capacities and electricity demand makes such a market incomplete. We follow a local risk minimization approach to price and hedge energy derivatives. Despite the richness of information included in the spot model, we obtain closed-form formulae for futures prices and semi-explicit formulae for spread options and European options on electricity forward contracts. An analysis of the electricity price risk premium is provided showing the contribution of demand and capacity to the futures prices. We show that when far from delivery, electricity futures behave like a basket of futures on fuels.
\end{abstract}

Keywords and phrases: Electricity spot and forward prices, fuels, capacity, electricity demand, scarcity function, local risk minimization, minimal martingale measure, power derivatives, spread options, extended incomplete Goodwin-Staton integral.

JEL Classification: D41; G13. AMS Classification (2000): 91B24; 91B26.

\section{Introduction}

This paper is a contribution to the development of electricity price models that can provide explicit or semi-explicit formulae for European derivatives on electricity markets. Since the beginning of the liberalization process of electricity markets in the 90s in Europe and in the USA, there has been an important research effort devoted to electricity price modeling for pricing derivatives. Due to the non-storable nature of electricity, it was - and still is - a challenge to reach a completely satisfying methodology that would suit the needs of trading desks: a realistic and robust model, computational tractability of prices and Greeks, consistency with market data. Two main standard approaches have usually been used to tackle this problem. The first one consists in directly modeling the forward curve dynamics and to deduce the spot price as a futures with immediate delivery. Belonging to this approach are, e.g., Clewlow and Strickland [2000] and Benth and Koekebakker [2007]. This approach is pragmatic in the sense that it models the prices of the available hedging instruments. However, it makes it difficult to capture the right dependencies between fuels and electricity prices (without cointegration). The second approach starts from a spot price model to deduce futures price as the expectation of the spot under a risk-neutral probability. The main benefit of this approach is that it provides a consistent framework for all possible derivatives. This approach has been successfully

\footnotetext{
${ }^{*}$ The authors thank the Finance and Sustainable Development Chair sponsored by EDF, CACIB and CDC for their support.

${ }^{\dagger}$ EDF R\&D and FiME, Finance for Energy Market Research Centre, www.fime-lab.org

${ }^{\ddagger}$ CEREMADE, University Paris-Dauphine \& FiME, Finance for Energy Market Research Centre. E-mail: campi@ceremade.dauphine.fr

$\S$ LPMA, University Paris 6-7 \& FiME, Finance for Energy Market Research Centre. E-mail: nicolas.langrene@etu.univ-paris-diderot.fr
} 
applied to commodities in [Schwartz, 1997] seminal work. Its main drawback is that it generally leads to complex computations for the prices of electricity derivatives. Inside this approach, most of the authors use an exogenous dynamics for the electricity spot price [Deng, 2000, Benth et al., 2003, Burger et al., 2004, Kolodnyi, 2004, Cartea and Figueroa, 2005, Benth et al., 2007, Benth and Vos, 2009, Goutte et al., 2009] and only a few try to deduce futures and option prices inside an equilibrium model or a model including a price formation mechanism [Pirrong and Jermakyan, 2000, Cartea and Villaplana, 2008, Pirrong and Jermakyan, 2008, Lyle and Elliott, 2009, Aïd et al., 2009].

The main contribution of this work is to provide analytical formulae for electricity futures and semiexplicit expressions for European options in an electricity spot price model that includes demand and capacities as well as fuel dynamics. Being able to model the dependencies between fuels and electricity is of great importance for spread options evaluation. To our knowledge, this is the first attempt performed in that direction.

Concerning the use of an equilibrium model or a price mechanism for pricing electricity derivatives, the closest works to ours can be found in [Pirrong and Jermakyan, 2000, 2008, Cartea and Villaplana, 2008, Lyle and Elliott, 2009]. It has been recognized that the mechanism leading to the electricity spot price was too complex to allow for a complete modeling that would fit the constraints of derivatives pricing. The simplest one is maybe Barlow [2002]'s model where the price is determined by the matching of a simple parametric offer curve and a random demand. Many authors have then derived reduced equilibrium models for electricity prices in this spirit [Kanamura and Ohashi, 2007, Coulon and Howison, 2009]. In Pirrong and Jermakyan [2000]'s work, electricity dependency on fuel prices is taken into account by modeling directly the dynamics of the marginal fuel. The authors manage to provide the partial differential equation and its boundary conditions for the price of a European derivative. The approach followed by Cartea and Villaplana [2008] and Lyle and Elliott [2009] is quite similar. Therein, the price is modeled as an exponential of a linear combination of demand and capacity. In general, it is difficult to introduce in the same framework the dependency of the electricity spot price from fuels and at the same time its dependency from demand and capacity. Dependency among fuels is generally captured by a simple correlation among Ornstein-Uhlenbeck processes as in Frikha and Lemaire [2009]'s paper or by cointegration method as in Benmenzer et al. [2007]'s work.

Here, we start from the marginal price model developed in [Aïd et al., 2009] and enrich it substantially to take into account the effect of the margin capacity uncertainty on futures prices. In order to include the biggest price spikes in our model, we introduce a multiplying factor allowing the electricity spot price to deviate from the marginal fuel price when demand gets closer to the capacity limit. Since electricity is a non-storable commodity, this factor accounts directly for the scarcity of production capacity. Although such an additional feature complexifies the model, we can still provide closed form formulae for futures prices. Under this model, any electricity futures contract behaves almost as a portfolio of futures contracts on fuels as long as the product is far from delivery. In contrast, near delivery, electricity futures prices are determined by the scarcity rent, i.e. demand and capacity uncertainties. The term scarcity is in line with the concept introduced by Geman and Nguyen [2005] since the multiplying factor is the inverse of the excess capacity which can be considered the analog of the inventory level for storable commodities. It is also consistent with Working's seminal observations on the relation between inventory and time spread price for agricultural commodities [Working, 1934, p. 206]. Moreover, the idea that electricity spot price spikes are related to a lack of capacity is some sort of common knowledge in the power system literature (see for instance Boogert and Dupont [2008]).

This paper is structured as follows. We first present the spot price model in Section 2, where we also perform the estimation of the scaling factor allowing the spot price to deviate from the marginal fuel price and comment on the production capacity scarcity effect. Then, in Section 3, we apply the spot model to pricing and hedging derivatives. We first choose realistic as well as tractable models for the dynamics of demand, capacities and fuel prices (Section 3.1). Then, since we work in an incomplete market setting, we discuss in Section 3.2 our choice for Föllmer and Schweizer [1991]'s Local Risk Minimization hedge criterion. Using such a criterion, a closed-form expression for futures prices is provided (Section 3.3) and semi-explicit formulae are given for spread options and for European options on futures (Section 3.4). Finally, an important part of this work is devoted to numerical simulations and backtesting presented in Section 4. Despite the apparent complexity of the model, the numerical computations essentially involve integration of functionals against Gaussian kernels. 
This part requires simple but long and tedious calculations. Thus, for the sake of readability, they have been relegated in Section B of the Appendix.

\section{$2 \quad$ Electricity spot market model}

\section{$2.1 \quad$ Spot model}

We denote by $P_{t}$ the electricity spot price at time $t$. At any time $t$, the electricity producer can choose, among $n$ possible fuels, the most convenient to produce electricity at that particular moment, called the marginal fuel. We will define the electricity spot price as a proportion of the spot price of the marginal fuel, corrected by a scarcity factor, ie. a factor depending on the current difference between available capacity and demand.

Denote as $S_{t}:=\left(S_{t}^{1}, \ldots, S_{t}^{n}\right)$ the fuel prices at time $t$, and $\left(h_{1}, \ldots, h_{n}\right)$ the corresponding heat rates, assumed to be constant. That means that $h_{i} S_{t}^{i}$ corresponds to the $t$-price of the quantity of $i$-th fuel necessary to produce $1 \mathrm{MWh}$ of electricity. Unlikely the model proposed in [Aïd et al., 2009], we make the further assumption that the production costs are ordered among fuels, ie. that $h_{1} S_{t}^{1} \leq \cdots \leq h_{n} S_{t}^{n}$. This ranking is supposed fixed and known. This assumption is realistic, at least when considering not too long maturities. Now, how does the electricity producer choose the most convenient fuel to use? For each $i=1, \ldots, n$, let $C_{t}^{i}>0$ denote the capacity of the $i$-th technology for electricity production at time $t$. Denote as $\bar{C}_{t}^{i}$ the total capacity at time $t$ of the first $i$ fuels, i.e. $\bar{C}_{t}^{i}:=\sum_{j \leq i} C_{t}^{j}$. For the maximal capacity $\bar{C}_{t}^{n}$ we will use, alternatively, the notation $C_{t}^{\max }$. We define the following production intervals:

$$
I_{t}^{1}:=\left(-\infty, C_{t}^{1}\right), \quad I_{t}^{i}:=\left[\bar{C}_{t}^{i-1}, \bar{C}_{t}^{i}\right), 2 \leq i \leq n-1, \quad I_{t}^{n}:=\left[\bar{C}_{t}^{n-1},+\infty\right),
$$

with the conventions that when $n=1$ there is only one interval $I_{t}^{1}:=\mathbb{R}$, and when $n=2$ there are only two intervals, the extreme ones, i.e. $I_{t}^{1}:=\left(-\infty, C_{t}^{1}\right)$ and $I_{t}^{2}:=\left[C_{t}^{1},+\infty\right)$. Thus, if the market demand at time $t$ for electricity $D_{t}$ belongs to the interval $I_{t}^{i}$, the last (marginal) unit of electricity is produced using the $i$-th fuel, when the corresponding plant is available. Otherwise, it is produced with the next fuel, more expensive, in the ranking. Having said that, we model the electricity spot price $P_{t}$ by the following relation:

$$
P_{t}=g\left(C_{t}^{\max }-D_{t}\right) \sum_{i=1}^{n} h_{i} S_{t}^{i} \mathbf{1}_{\left\{D_{t} \in I_{t}^{i}\right\}}, \quad t \geq 0
$$

where we recall that $C_{t}^{\max }=\bar{C}_{t}^{n}=\sum_{i=1}^{n} C_{t}^{i}$ is the maximal capacity of the whole system at time $t$, and $g$ is a bounded real-valued function given by:

$$
g(x)=\min \left(M, \frac{\gamma}{x^{\nu}}\right) \mathbf{1}_{\{x>0\}}+M \mathbf{1}_{\{x \leq 0\}}
$$

where $\gamma, M$ and $\nu$ are positive and constant parameters.

The term $C_{t}^{\max }-D_{t}$ is the margin capacity of the system. It is an indicator of the tension in the system due to scarcity, since electricity is non-storable, and the term $g\left(C_{t}^{\max }-D_{t}\right)$ models the effect of this tension to prices. This is why we will call $g$ the scarcity function of electricity prices. The margin capacity is better at capturing electricity price spikes better than demand itself as in Barlow [2002] and Kanamura and Ohashi [2007] models. This remark has already been pointed out by several authors as, e.g., Cartea and Villaplana [2008], Coulon and Howison [2009] and Lyle and Elliott [2009]. Coulon and Howison [2009] provide the most complex framework, which is based on a model for the bidding curves. Unfortunately their model does not allow for analytical expressions of futures prices as soon as there are more than two fuels. On the contrary, in Cartea and Villaplana [2008] and Lyle and Elliott [2009]'s works, drastic simplifications of the electricity market rules allow them to provide analytical expressions for futures prices, and, in Lyle and Elliott [2009]'s model, for European option prices as well. In their models, the electricity spot price is expressed as an exponential of the margin capacity, which simplifies considerably the computation of derivative prices and of the risk premium. 
However, the exponential function does not enable such sharp price spikes as those observable on electricity spot data. On the contrary, as it will be shown below, our choice (2.3) of a power law of margin capacity can accurately reproduce such a behaviour, even for smooth and rather simple dynamics of demand and capacity processes.

\section{$2.2 \quad$ Estimation and backtesting}

Before making further use of our model (2.2), we aim in this section at assessing its accuracy on historical data. In particular, a methodology to estimate the scarcity function (2.3) will be proposed.

\subsubsection{Data set}

We choose to test the model on the French deregulated power market. We retrieve the required data from the following sources:

- PowerNext for the hourly power spot price $P_{t}$.

- RTE, the French transmission system operator, for the hourly power demand $D_{t}$ and capacity $C_{t}^{i}$ for each fuel.

- TFS (Tradition Financial Services) for the daily coal price (API\#2).

- IPE (International Petroleum Exchange) for the daily oil price (Brent).

- ECB (the European Central Bank) for the daily USD/EUR exchange rate, to convert the coal and oil prices, which are denominated in US dollars, into euros.

- ECX (European Climate Exchange) for the daily $\mathrm{CO}_{2}$ price.

- EDF, French power utility, for heat rates $h_{i}$ and $\mathrm{CO}_{2}$ emission rates.

We focus our analysis on one particular hour of the day, namely the $19^{\text {th }}$, which usually bears the highest demand level of a day. As a consequence, during these peak hours, the electricity price is almost always fixed by one of the two most expensive technologies that are coal and oil. Consequently, such a choice simplifies the model since it makes possible to work with only $n=2$ technologies.

Thus, $S_{t}^{1}$ corresponds to the daily coal price, converted to EUR/MWh using the USD/EUR exchange rate and the coal heat rate $h_{1}$. We include the price of $\mathrm{CO}_{2}$ emissions in $S_{t}^{1}$, using the daily $\mathrm{CO}_{2}$ price and the emission rate of French coal plants. Similarly, $S_{t}^{2}$ corresponds to the daily overall oil price.

For the simplification to $n=2$ technologies to hold, it suffices to define $D_{t}$ as the residual demand corresponding to coal and oil. This quantity can be extracted from RTE demand, production and capacity data. More details are given in Appendix A.

Our dataset covers the period going from November $13^{\text {th }}, 2006$ to April $30^{\text {th }}, 2010$. The beginning of the period was fixed by the availability of the production capacity data. On this period, during the $19^{\text {th }}$ hours, the average electricity spot price was $74.5 € / \mathrm{MWh}$ (see Figure $2.2 \mathrm{a}$ ), the average coal price (including heat rate and $\mathrm{CO}_{2}$ ) was $47.4 € / \mathrm{MWh}$, and the average oil price (including heat rate and $\mathrm{CO}_{2}$ ) was $102.0 € / \mathrm{MWh}$.

\subsubsection{Parameters estimation}

So far, we have retrieved all the necessary data to test equation (2.2). The last remaining step is to estimate the parameters $M, \gamma$ and $\nu$ that characterize the scarcity function (2.3) recalled below:

$$
g(x)=\min \left(M, \frac{\gamma}{x^{\nu}}\right) \mathbf{1}_{\{x>0\}}+M \mathbf{1}_{\{x \leq 0\}}
$$


First, we fix $M$ so as to roughly match the high cap on electricity spot price, defined by the market as $3000 € / \mathrm{MWh}^{1}$. Our estimate is $M=30$.

Now, we turn our attention to the parameters $\gamma$ and $\nu$. Remark first that $\gamma$, unlike $M$ and $\nu$, depends on the unit in which $D_{t}$ and $C_{t}^{i}, 1 \leq i \leq n$, are denominated. This is a consequence of the following results:

Proposition 2.1. (Change of Unit) Let $N>0$ be a constant. The following holds:

$$
P_{t}\left(C_{t}, D_{t}, S_{t}, M, \gamma, \nu\right)=P_{t}\left(\frac{C_{t}}{N}, \frac{D_{t}}{N}, S_{t}, M, \frac{\gamma}{N^{\nu}}, \nu\right)
$$

where we have explicited the parameters involved in the definition of $P_{t}$.

Proof. First, one can easily check that $g(x ; M, \gamma, \nu)=g\left(\frac{x}{N} ; M, \frac{\gamma}{N^{\nu}}, \nu\right)$. The result follows then from equation (2.2) and the fact that $\left\{\frac{D_{t}}{N} \in \frac{I_{t}^{i}}{N}\right\}=\left\{D_{t} \in I_{t}^{i}\right\}$ a.s., $1 \leq i \leq n$.

This useful change of unit formula indicates that we are free to choose the desired unit for $D_{t}$ and $C_{t}$. In our case, we choose to convert these data, which are provided in MWh, into GWh $(N=1000)$, and to estimate the corresponding "GWh- $\gamma$ ". This renormalisation will prove to be numerically convenient in Section 4.2.

Going back to the estimation of $\nu$ and $\gamma$, Figure 2.1a depicts the quantity $y_{t}:=\frac{P_{t}}{\sum_{i=1}^{n} h_{i} S_{t}^{i} \mathbf{1}_{\left\{D_{t} \in I_{t}^{i}\right\}}}$ as a function of $x_{t}:=C_{t}^{\max }-D_{t}$ for the period we consider, i.e. November $13^{\text {th }}, 2006$ to April $30^{\text {th }}, 2010$. The quantity $y_{t}$ corresponds to the price of electricity corrected from the effect of the marginal fuel price, while $x_{t}$ corresponds to the remaining available production capacity (margin capacity). Recalling equation (2.2), the relation between $x_{t}$ and $y_{t}$ is to be captured by $g$. Observe that the highest prices (high $y_{t}$ ) occur mostly when there is not much available capacity left (low $x_{t}$ ), which simply translates the law of supply and demand. It suggests a decreasing causality relationship between $x_{t}$ and $y_{t}$.

First, note that as the high cap price is not reached in our time period, the parameter $M$ will not intervene in the following. Now, remark that such classical tools as least squares or maximum likelihood are not adapted to the estimation of $g$. Indeed, both $x_{t}$ and $y_{t}$ are random, and the slope of the relation between $x_{t}$ and $y_{t}$ becomes extremely steep as $x_{t}$ approaches zero. These tools, that measure the error in one dimension only $\left(y_{t}\right)$ are bound to miss much of the slope part. To overcome this difficulty, our idea is intuitively the following: if a strictly decreasing deterministic relation between $x_{t}$ and $y_{t}$ was to be inforced, then a similar relation would link their quantiles, from which the estimation would be easier. This is the content of the following proposition:

Proposition 2.2. Let $X$ and $Y$ be two real random variables on the probability space $(\mathbb{R}, \mathcal{B}(\mathbb{R}), \mathbb{P})$, where $\mathcal{B}(\mathbb{R})$ denotes the corresponding Borel $\sigma$-field. Assume that both $X$ and $Y$ have continuous and strictly increasing cumulative distribution functions. Thus, their quantile functions $q_{X}$ and $q_{Y}$ exist, are unique, and defined for all $p \in[0,1]$ by:

$$
\mathbb{P}\left(X \leq q_{X}(p)\right)=p \quad, \quad \mathbb{P}\left(Y \leq q_{Y}(p)\right)=p
$$

Suppose that there exists a strictly decreasing function $h$ such that the relation $Y=h(X)$ holds $\mathbb{P}$-as. Then for all $p \in[0,1]$ :

$$
q_{Y}(1-p)=h\left(q_{X}(p)\right)
$$

Proof. For every $p \in[0,1]$ :

$$
\begin{aligned}
& 1-p=1-\mathbb{P}\left(X \leq q_{X}(p)\right)=1-\mathbb{P}\left(h(X) \geq h\left(q_{X}(p)\right)\right)=\mathbb{P}\left(Y \leq h\left(q_{X}(p)\right)\right) \\
& 1-p=\mathbb{P}\left(Y \leq q_{Y}(1-p)\right)
\end{aligned}
$$

Thus $\mathbb{P}\left(Y \leq q_{Y}(1-p)\right)=\mathbb{P}\left(Y \leq h\left(q_{X}(p)\right)\right)$, and the unicity of the quantile function yields $q_{Y}(1-p)=$ $h\left(q_{X}(p)\right)$. 
g(.) estimation data

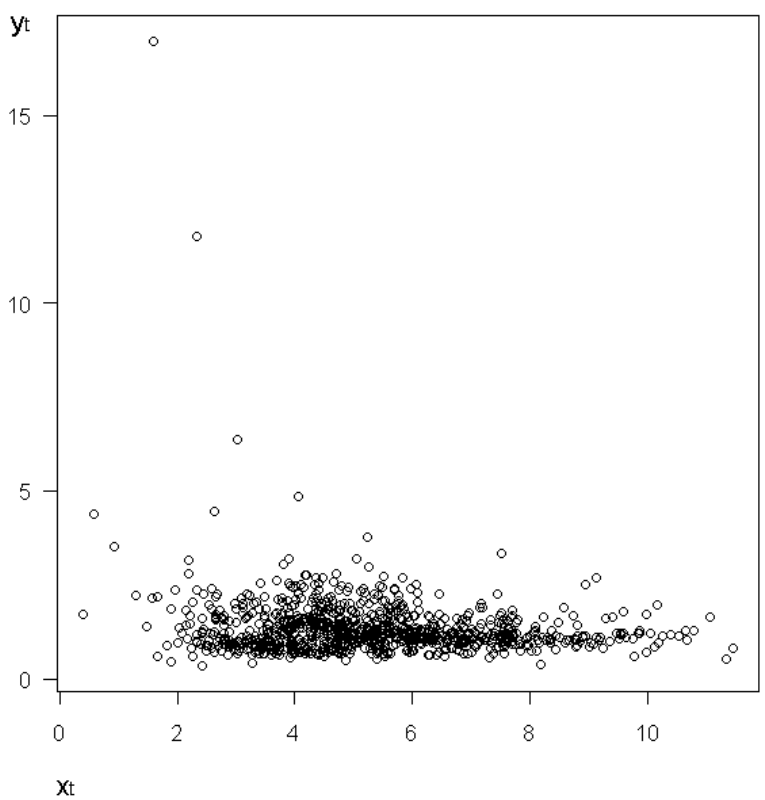

(a) Data g(.) estimation

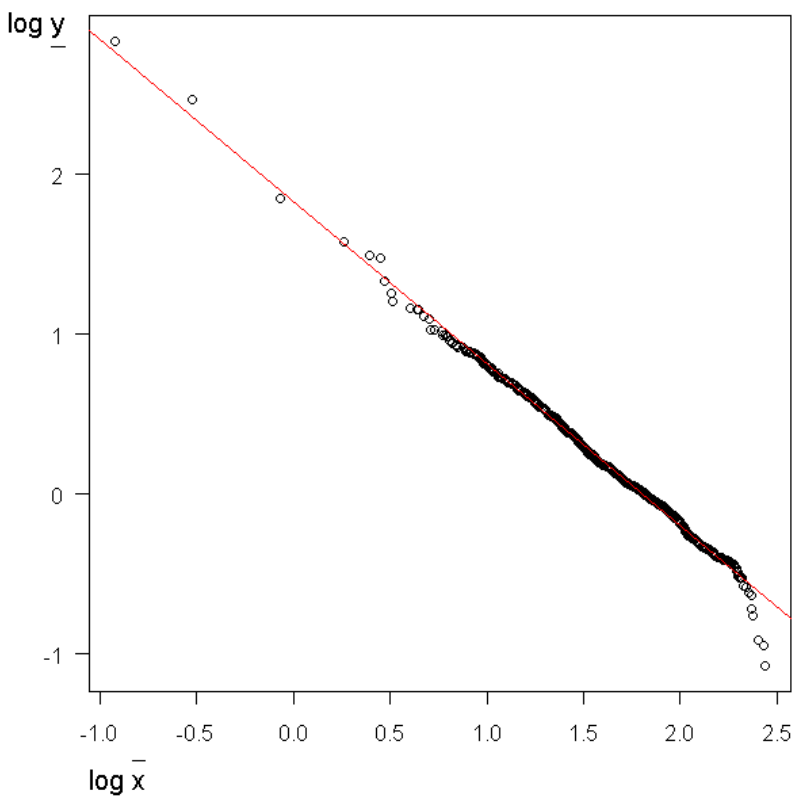

(b) Regression of $\log (\underline{y})$ versus $\log (\bar{x})$

Figure 2.1: $g$ estimation

Consequently, if one assumes a strictly decreasing deterministic relation $h$ between $x_{t}$ and $y_{t}$, then $h$ can be estimated from the quantiles of $x_{t}$ and $y_{t}$. This will prove to be very simple and much more robust and accurate than working directly with the realizations of $x_{t}$ and $y_{t}$.

Denote as $m$ the sample size, and $\left(x_{1}, y_{1}\right), \ldots,\left(x_{m}, y_{m}\right)$ the realizations of $\left(x_{t}, y_{t}\right)$. Define the sorted vectors $\bar{x}:=\left(\bar{x}_{1}, \ldots, \bar{x}_{m}\right)$ and $\underline{y}:=\left(\underline{y}_{1}, \ldots, \underline{y}_{m}\right)$ in such a way that $\bar{x}_{1} \leq \ldots \leq \bar{x}_{m}$ (increasing order) and $\underline{y}_{1} \geq \ldots \geq \underline{y}_{m}$ (decreasing order). The last step is to choose some $p \in[0,1]$ and to estimate the quantiles $q_{Y}(p)$ and $q_{X}(1-p)$. A simple and natural choice is to select $p_{i}:=\frac{i}{m}, 1 \leq i \leq m$, and to choose as quantile estimator for $q_{X}\left(p_{i}\right)$ and $q_{Y}\left(1-p_{i}\right)$ the inverse of the empirical cumulative distribution function, which yield in this case the estimates $\bar{x}_{\left\lceil m p_{i}\right\rceil}=\bar{x}_{i}$ and $\underline{y}_{\left\lceil m p_{i}\right\rceil}=\underline{y}_{i}(\lceil x\rceil$ indicates the upper integer part of any real number $x)$. Figure $2.1 \mathrm{~b}$ represents the quantity $\log (y)$ as a function of $\log (\bar{x})$.

A strikingly accurate linear relation appears. In particular it retranscribes very well the highest peak prices, which advocates a power-law description of electricity price spikes. The very lowest prices seem not to fit the linear relation. However a specific analysis of these few points reveals that they correspond to the few holidays (hence low demand) of the period considered for which the marginal fuel was gas during the $19^{\text {th }}$ hour of the day. As we neglected on purpose this possibilily to work with only two fuels (coal and oil), the consequence is that the residual capacity is undervalued at these specific dates, breaking the smooth relation. Otherwise, the linear relation appears very plausible.

Denote as $-\nu$ and $\log (\gamma)$ the coefficients of the regression, ie. $\log (y)=-\nu \log (\bar{x})+\log (\gamma)$. Taking the exponential yields $\underline{y}=\frac{\gamma}{\bar{x}^{\nu}}$. Thus our estimation of the relation between the quantiles $q_{y_{t}}(1-p)$ and $q_{x_{t}}(p)$ is given by $h(x)=\frac{\gamma}{x^{\nu}}$. Numerically, we found the estimates $\nu=1.022$ and $\gamma=6.241$ with the corresponding $95 \%$ confidence intervals of $[1.017,1.028]$ and $[6.186,6.296]$ respectively, and an adjusted $R$-squared equal to 0.9935 (see Figure $2.1 \mathrm{~b}$ ).

Now, using Proposition 2.2, we can trace back to the relation between $y_{t}=\frac{P_{t}}{\sum_{i=1}^{n} h_{i} S_{t}^{i} \mathbf{1}_{\left\{D_{t} \in I_{t}^{i}\right\}}}$ and $x_{t}=C_{t}^{\max }-D_{t}$. Indeed, the consequence of Proposition 2.2 combined to the empirical power law

\footnotetext{
${ }^{1}$ see http://www.epexspot.com/en/product-info/auction/france
} 
relation between $\bar{x}$ and $\underline{y}$ is the following: if one assumes a strictly decreasing deterministic relation between $x_{t}$ and $y_{t}$, then it ought be a power law relation, defined by $h(x)=\frac{\gamma}{x^{\nu}}$. This, combined with the modeling of the market cap price, leads to the relation (2.3) and to our model (2.2). Therefore, our estimation of the parameters $\nu$ and $\gamma$ provides also empirical evidence for the relevance of our spot model.

\subsubsection{Backtest}

Finally, with our data and our estimated parameters, we can backtest our model, i.e. we can compute the quantity $g\left(C_{t}^{\max }-D_{t}\right) \sum_{i=1}^{n} h_{i} S_{t}^{i} \mathbf{1}_{\left\{D_{t} \in I_{t}^{i}\right\}}$ for each date $t$ of our dataset, and compare it to the realized electricity spot prices for the same dates. Figure 2.2a illustrates this comparison.

Spot Backtest

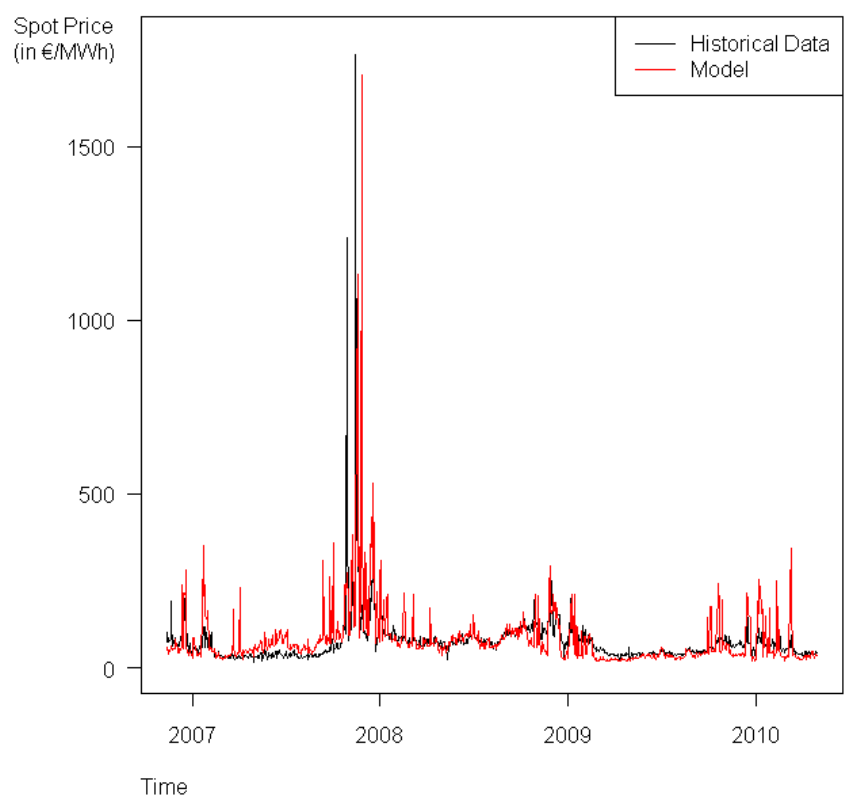

(a) Spot model vs. Historical prices

\section{Model comparison}

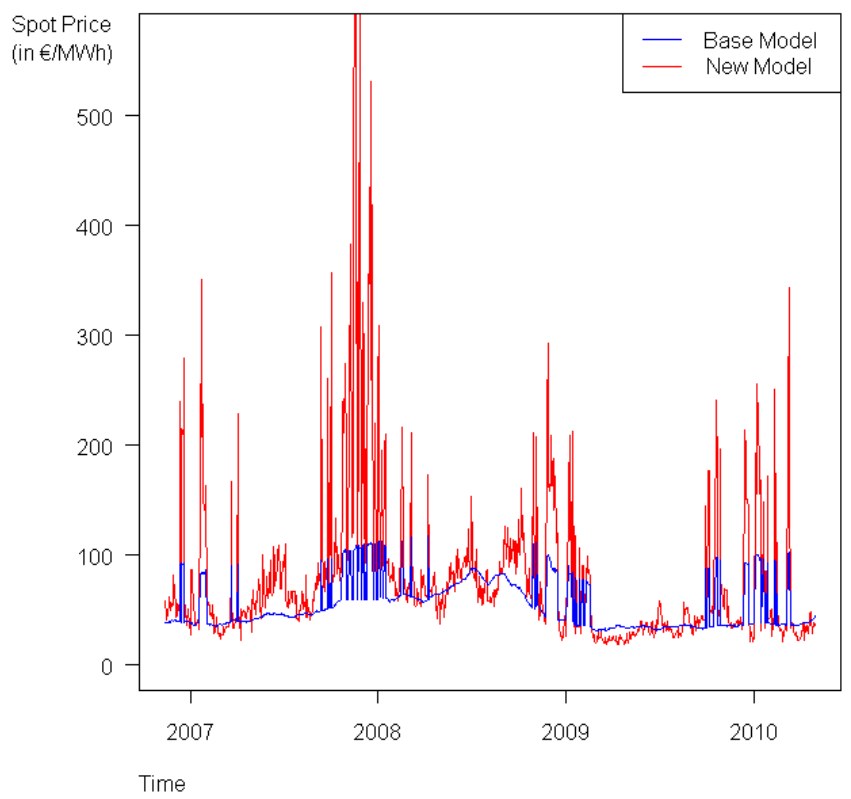

(b) Spot model vs. Marginal fuel price model

Figure 2.2: Backtests

It can be seen that the base prices are described rather well by the model (thanks to the marginal price $\sum_{i=1}^{n} h_{i} S_{t}^{i} \mathbf{1}_{\left\{D_{t} \in I_{t}^{i}\right\}}$ ) and that the model is able to produce price peaks of relatively good size (thanks to the multiplication to the scarcity function $g$ and its power law shape (2.3)) and timing (thanks to the choice of the margin capacity $C_{t}^{\max }-D_{t}$ as a state variable). It is these adequate prisms $C_{t}^{\max }-D_{t}$ and $\sum_{i=1}^{n} h_{i} S_{t}^{i} \mathbf{1}_{\left\{D_{t} \in I_{t}^{i}\right\}}$ that enable $g$ to be stationary over time, making the model robust.

Figure $2.2 \mathrm{~b}$ compares the model $(2.2)$ to the simpler model $\sum_{i=1}^{n} h_{i} S_{t}^{i} \mathbf{1}_{\left\{D_{t} \in I_{t}^{i}\right\}}$ (i.e. when $g \equiv 1$ ), with the price scale limited to $600 € / M W h$ for readability. As one can see, both models behave similarly as long as $C_{t}^{\max }-D_{t}$ is large, but the simpler model is unable to produce large price spikes in periods of tension (i.e. when $C_{t}^{\max }-D_{t}$ is low). This lack is corrected by the present model (2.2).

Remark 2.3. The estimation problem studied in this Section (see Figure 2.1a) is related to the problem of estimating the electricity supply curve. The usual approach is to propose a parametric modeling of this curve (or of a variant of this curve) and to estimate the corresponding parameters via such methods as maximum likelihood (Barlow [2002], Cartea and Villaplana [2008], Coulon and Howison [2009]), non-linear regression (Lyle and Elliott [2009]) or non-linear least squares (Kanamura and Ohashi [2007]). In Pirrong and Jermakyan [2000], a semi-parametric estimation is performed using local quadratic regression. In our case, the supply curve considered is corrected from the marginal 
fuel price (as in Pirrong and Jermakyan [2000]), its input is the difference $C_{t}-D_{t}$ (unlike the more common choices of $D_{t}$ or $D_{t} / C_{t}$ ), and the distinctive feature of our estimation procedure is that it is performed on the quantiles of the inputs, which, it turns out, allows for a simple estimation using linear regression. Consequently, as they were not needed, other estimation techniques were not investigated. However, it cannot be excluded that more involved estimation techniques may be required, for instance, to apply our model to other specific electricity markets. For the time being, we leave these empirical investigations and extensions for further research.

\section{Pricing and hedging}

In this section, we use the model (2.2) to derive pricing and hedging formulae for power derivatives, including forward contracts. We first model the different processes involved in the equation (2.2), namely the capacity processes $C_{t}^{i}$, the electricity demand $D_{t}$ and the fuel prices $S_{t}^{i}, 1 \leq i \leq n$. The fuels $S^{i}$ are clearly tradable, but demand and capacities $D$ and $C^{i}$ are not, which means that we are going to work in an incomplete market setting. Consequently, a perfect hedging will not be possible, and, equivalently, the market will have infinitely many Equivalent (local) Martingale Measures (henceforth EMM). Many criteria for the choice of a 'good' EMM are available in the literature. In this paper, we use the local risk minimization criterion introduced by Föllmer and Schweizer [1991], which is based on a financially meaningful decomposition of contingent claims into hedgeable and non-hedgeable parts and gives rise to explicit price formulae, as we will see next. Investigation of other criteria such as, e.g., utility indifference pricing or mean-variance hedging, and their comparison are left for future research (one can refer to [Bouchard and Nguyen Huu, 2010] and [Goutte et al., 2009] for applications of these other criteria to electricity markets).

\subsection{Model for capacity, demand and fuel prices}

Let $(\Omega, \mathbb{P}, \mathcal{F})$ be a given probability space, where $\mathbb{P}$ is the historical (or statistical) probability measure. $\mathbb{E}$ will denote the expectation operator taken with respect to $\mathbb{P}$. All the subsequent processes, namely $C, D$ and $S$, will be defined on this probability space. The market filtration $\mathcal{F}_{t}$ will be the natural filtration generated by all Brownian motions driving the dynamics of all such processes. We assume from the beginning that the spot interest rate $r$ is a positive constant. For the sake of simplicity, we set storage cost and convenience yield of every fuel equal to zero.

Market of fuels. We recall from Section 2 that for $1 \leq i \leq n, S_{t}^{i}$ denotes the price of fuel $i$ at time $t$, that $h_{i}$ denotes the corresponding heat rate, such that $h_{i} S_{t}^{i}$ is expressed in units of currency per MWh, and that we assume a fixed order $h_{1} S_{t}^{1} \leq \cdots \leq h_{n} S_{t}^{n}$ between fuels. In order to enforce this assumption, we model the dynamics of the fuel spreads $Y_{t}^{i}:=h_{i} S_{t}^{i}-h_{i-1} S_{t}^{i-1}$ (with the convention $\left.S^{0} \equiv 0\right)$ rather than the dynamics of the fuels directly. We choose $Y=\left(Y^{1}, \ldots, Y^{n}\right)$ to be a vector of independent geometric Brownian motions under the statistical measure $\mathbb{P}$, i.e. for $1 \leq i \leq n$ :

$$
d Y_{t}^{i}=\mu_{i} Y_{t}^{i} d t+\sigma_{i} Y_{t}^{i} d W_{t}^{i}, \quad Y_{0}^{i}>0
$$

where $W=\left(W^{1}, \ldots W^{n}\right)$ is a $n$-dimensional Brownian motion for the measure $\mathbb{P}$ and $\mu_{i}, \sigma_{i}(1 \leq i \leq n)$ are fixed real numbers with $\sigma_{i}>0 . \mathcal{F}^{W}=\left(\mathcal{F}_{t}^{W}\right)$ denotes the natural (and P-saturated) filtration generated by $W$. Note that $h_{i} S^{i}=\sum_{j \leq i} Y^{j}$ and, as a consequence of the positivity of geometric Brownian motions, the condition $h_{i} S^{1}<\cdots<h_{n} S^{n}$ is satisfied.

The $\mathbb{P}$-dynamics of $S^{i}, 1 \leq i \leq n$, is then given by:

$$
d S_{t}^{i}=\frac{1}{h_{i}} \sum_{j \leq i}\left(\mu_{j} Y_{t}^{j} d t+\sigma_{j} Y_{t}^{j} d W_{t}^{j}\right) .
$$

Since each $S_{t}^{i}$ is a.s. positive, we can rewrite its $\mathbb{P}$-dynamics in the following way:

$$
\frac{d S_{t}^{i}}{S_{t}^{i}}=\mu_{t}^{S, i} d t+\sigma_{t}^{S, i} d W_{t}^{S, i}
$$


where the drift $\mu_{t}^{S, i}$, the volatility $\sigma_{t}^{S, i}$ and the Brownian motions $W^{S, i}$ are given by:

$$
\mu_{t}^{S, i}=\sum_{j \leq i} \frac{Y_{t}^{j}}{h_{i} S_{t}^{i}} \mu_{j}, \quad \sigma_{t}^{S, i}=\sqrt{\sum_{j \leq i}\left(\frac{Y_{t}^{j}}{h_{i} S_{t}^{i}}\right)^{2} \sigma_{j}^{2}}, \quad d W_{t}^{S, i}=\frac{1}{\sigma_{t}^{S, i}} \sum_{j \leq i} \frac{Y_{t}^{j}}{h_{i} S_{t}^{i}} \sigma_{j} d W_{t}^{j}
$$

To verify that $W^{S, i}$ is a Brownian motion it suffices to check that $\left\langle W^{S, i}\right\rangle_{t}=t$ and use Lévy's characterization theorem to conclude. Notice that $\mathcal{F}^{W}=\mathcal{F}^{W^{S}}$, where $\mathcal{F}^{W^{S}}$ denotes the natural filtration generated by the $n$-dimensional process $W^{S}:=\left(W^{S, 1}, \ldots, W^{S, n}\right)$.

Electricity demand and production capacities. The demand for electricity is modelled by a process $D$, adapted to the natural filtration $\mathcal{F}_{t}^{D}$ generated by a Brownian motion $W^{D}$ under $\mathbb{P}$. Similarly, the production capacities from each fuel are modelled by processes $C^{i}, 1 \leq i \leq n$, adapted to the natural filtration $\mathcal{F}_{t}^{C}$ generated by an $n$-dimensional Brownian motion $W^{C}=\left(W^{C, 1}, \ldots, W^{C, n}\right)$ under $\mathbb{P}$. We assume the following dynamics:

$$
\begin{aligned}
d D_{t} & =a\left(t, D_{t}\right) d t+b\left(t, D_{t}\right) d W_{t}^{D} \\
d C_{t}^{i} & =\alpha_{i}\left(t, C_{t}^{i}\right) d t+\beta_{i}\left(t, C_{t}^{i}\right) d W_{t}^{C, i}
\end{aligned}
$$

where $a, b, \alpha_{i}, \beta_{i}: \mathbb{R}_{+} \times \mathbb{R} \mapsto \mathbb{R}$ are measurable functions such that the SDEs (3.2) and (3.3) admit unique strong solutions on, respectively, $\mathcal{F}^{D}$ and on the natural filtration generated by $W^{C, i}{ }^{1}$ We make the following standing assumption.

Assumption 3.1. We assume that the Brownian motions $W, W^{C}$ and $W^{D}$ are mutually independent under the true probability $\mathbb{P}$. Moreover, the market filtration, denoted $\left(\mathcal{F}_{t}\right)$, is the natural filtration satisfying usual conditions - generated by all these Brownian motions, i.e. $\mathcal{F}_{t}=\mathcal{F}_{t}^{S} \vee \mathcal{F}_{t}^{C} \vee \mathcal{F}_{t}^{D}$.

\subsection{Choice of the pricing measure}

\subsubsection{Some preliminaries on local risk minimization}

We recall some basic facts on local risk minimization (henceforth LRM) approach for pricing and hedging in incomplete markets. This approach has been introduced by Föllmer and Schweizer [1991]. We will essentially follow the two survey papers by Pham [2000] and Schweizer [2001]. All the processes we will introduce in this section refer to a given filtration $\left(\mathcal{F}_{t}\right)$ satisfying usual conditions and representing the market's information flow.

Let $X$ be a discounted continuous price process, i.e. $X$ is an adapted continuous $\mathbb{R}^{d}$-valued semimartingale with decomposition $X=X_{0}+M+A$, where $X_{0}$ is a constant in $\mathbb{R}^{d}, M$ is a local martingale and $A$ a finite variation process such that $M_{0}=A_{0}=0$. We assume that there exists a square integrable EMM $\mathbb{Q}$ for $X$, i.e. $X$ is a local martingale under $\mathbb{Q}$ with $d \mathbb{Q} / d \mathbb{P} \in L^{2}(\mathbb{P})$. It is well known that under such an assumption, the finite variation part $A$ is absolutely continuous with respect to $M$ 's quadratic variation, i.e. it satisfies

$$
A_{t}=\int_{0}^{t} d\langle M\rangle_{s} \lambda_{s}, \quad t \in[0, T]
$$

for some predictable $\mathbb{R}^{n}$-valued process $\lambda$. A portfolio strategy is a pair $\varphi=(V, \vartheta)$ where $V$ is a real-valued adapted process such that $V_{T} \in L^{2}(\mathbb{P})$ and $\vartheta$ is a predictable, $\mathbb{R}^{d}$-valued, $X$-integrable process such that $\int_{0}^{T} \vartheta_{t} d X_{t} \in L^{2}(\mathbb{P})$ and $\int \vartheta d X$ is a $Q$-martingale for all $Q \in \mathcal{M}_{2}^{e}$, the set of all $\mathbb{P}$-equivalent probability measures with square integrable derivative and making $X$ a local martingale. The set of all such strategies $\vartheta$ will be denoted by $\Theta$.

We now associate to each portfolio strategy $\varphi=(V, \vartheta)$ a process, which will be very useful in the sequel in describing the main features of the LRM approach: the cost process $\operatorname{Cost}(\varphi)$. The cost process of a portfolio strategy $\varphi=(V, \vartheta)$ is defined by:

$$
\operatorname{Cost}_{t}(\varphi)=V_{t}-\int_{0}^{t} \vartheta_{u} d X_{u}, \quad t \in[0, T]
$$

\footnotetext{
${ }^{1}$ See, e.g., [Karatzas and Shreve, 1991] or [Revuz and Yor, 1991] for standard assumptions ensuring such properties.
} 
A portfolio strategy $\varphi$ is called self-financing if its cost process $\operatorname{Cost}(\varphi)$ is constant $\mathbb{P}$ a.s.. It is called mean self-financing if $\operatorname{Cost}(\varphi)$ is a martingale under $\mathbb{P}$. Let $H$ be a square-integrable, $\mathcal{F}_{T^{-}}$ measurable contingent claim. We say that a portfolio strategy $\varphi=(V, \vartheta)$ is $H$-admissible if $V_{T}=H$, $\mathbb{P}$ a.s.. Therefore, an $H$-admissible portfolio strategy $\varphi$ is called locally risk minimizing (henceforth LRM-strategy) if the corresponding cost process $\operatorname{Cost}(\varphi)$ belongs to $\mathbb{H}^{2}(\mathbb{P})$ (the Banach space of all $\mathbb{P}$-martingales bounded in $L^{2}(\mathbb{P})$, equipped with the norm $\left.\|M\|^{2}=\mathbb{E}\left[\sup _{t}\left|M_{t}\right|\right]^{2}\right)$ and is orthogonal to $X$ under $\mathbb{P}$. There exists a LRM-strategy if and only if $H$ admits a decomposition:

$$
H=H_{0}+\int_{0}^{T} \vartheta_{t}^{H} d X_{t}+L_{T}^{H}, \quad \mathbb{P} \text { a.s. },
$$

where $H_{0}$ is a constant, $\vartheta^{H} \in \Theta$ and $L^{H} \in \mathbb{H}^{2}(\mathbb{P})$ is orthogonal to $X$. Such a decomposition is called the Föllmer-Schweizer decomposition of $H$ under $\mathbb{P}$, and the portfolio strategy $\varphi=\left(V, \vartheta^{H}\right)$ with

$$
V_{t}=H_{0}+\int_{0}^{t} \vartheta_{s}^{H} d X_{s}+L_{t}^{H}, \quad \mathbb{P} \text { a.s. }, \quad t \in[0, T] .
$$

is a LRM-strategy for $X$. There is a very useful characterization of the LRM-strategy by means of the Galtchouk-Kunita-Watanabe decomposition (henceforth GKW-decomposition) of $H$ under a suitable EMM, namely the minimal EMM introduced by Föllmer and Schweizer [1991]. We recall now some basic facts about this measure and its deep relation with the LRM approach. We denote by $\widehat{Z}$ the minimal martingale density:

$$
\widehat{Z}_{t}=\mathcal{E}\left(-\int \lambda d M\right)_{t}, \quad t \in[0, T]
$$

Since Delbaen et al. [1997] we know that the existence of a Föllmer-Schweizer decomposition (and so of a unique LRM-strategy) for every $H \in L^{2}\left(\mathbb{P}, \mathcal{F}_{t}\right)$, for any $t \in[0, T]$, is equivalent to assuming an additional integrability condition on $\widehat{Z}$, which is usually called $R_{2}(\mathbb{P}$ ) (see Delbaen et al. [1997] for details). Such a condition will be verified in our model. Moreover, under such a condition, we can define on $\mathcal{F}_{t}$, for all $t \in[0, T]$, an EMM $\widehat{\mathbb{Q}}$ for $X$, given by:

$$
\left.\frac{d \widehat{\mathbb{Q}}}{d \mathbb{P}}\right|_{\mathcal{F}_{t}}=\widehat{Z}_{t}
$$

which is called minimal $E M M$ for $X$. We will denote $\widehat{\mathbb{E}}$ the expectation operator under $\widehat{\mathbb{Q}}$. We now quote without proof (for which we refer to Föllmer and Schweizer [1991], Theorem 3.14, p. 403) the following fundamental result relating the minimal EMM and the LRM-strategy:

Theorem 3.2. Let $H$ be a contingent claim in $L^{2}\left(\mathbb{P}, \mathcal{F}_{T}\right)$. The LRM-strategy $\widehat{\varphi}$, hence also the corresponding Föllmer-Schweizer decomposition (3.4), is uniquely determined. It can be computed in terms of the minimal EMM $\widehat{\mathbb{Q}}:$ if $\widehat{V}_{t}^{H}, t \in[0, T]$, denotes a right-continuous version of the $\widehat{\mathbb{Q}}-$ martingale $\widehat{\mathbb{E}}\left[H \mid \mathcal{F}_{t}\right], t \in[0, T]$, with $G K W$-decomposition

$$
\widehat{V}_{t}^{H}=\widehat{V}_{0}^{H}+\int_{0}^{t} \widehat{\vartheta}_{s}^{H} d X_{s}+\widehat{L}_{t}^{H}, \quad t \in[0, T],
$$

then the portfolio strategy $\widehat{\varphi}^{H}=\left(\widehat{V}^{H}, \widehat{\vartheta}^{H}\right)$ is the LRM-strategy for $H$ and its cost process is given by $\operatorname{Cost}(\widehat{\varphi})=\widehat{\mathbb{E}}[H]+\widehat{L}^{H}$.

This theorem gives us a practical way for computing the LRM-strategy of a given contingent claim $H$. Indeed, in order to find its LRM-strategy and the associated cost process, we need to compute only its GKW-decomposition and identify the integral part and the orthogonal part. The integral part represents the hedgeable part of the claim $H$, while the orthogonal part $L^{H}$ represents the unhedgeable part or residual risk. The expectation of $H$ under the minimal EMM $\widehat{\mathbb{Q}}, \widehat{\mathbb{E}}[H]$, is clearly one of the infinitely many no-arbitrage prices of $H$ and it can be also viewed as the initial wealth allowing to hedge the hedgeable part of $H$. Moreover, in Hobson [2005], it is shown that in a large class of diffusion market models with non-tradable assets, the expectation of a contingent claim $H$ under the minimal EMM, i.e. $\widehat{\mathbb{E}}[H]$, is an upper bound for bid utility indifference prices of $H$. In the next sections, we will use this approach to price and (partially) hedge power derivatives. 


\subsubsection{LRM in our energy market model}

We have already noticed that our market model is incomplete. Thus it admits infinitely many EMMs. Here is a complete description of such measures.

Consider first the submarket composed of fuels only. Since each $Y^{i}$ admits a unique EMM $\mathbb{Q}^{i}$ and their corresponding Radon-Nikodym derivatives are independent, it is easy to see that the measure $\widehat{\mathbb{Q}}$ defined as the product of all the $\mathbb{Q}^{i}$ 's is an EMM for $S$. More precisely,

$$
\frac{d \widehat{\mathbb{Q}}}{d \mathbb{P}}=\prod_{i=1}^{n} \frac{d \mathbb{Q}^{i}}{d \mathbb{P}}=\prod_{i=1}^{n} e^{-\lambda_{i} W_{T}^{i}-\frac{\lambda_{i}^{2}}{2} T}, \quad \text { on } \mathcal{F}_{T}
$$

where $\lambda_{i}=\frac{\mu_{i}-r}{\sigma_{i}}$ is the market price of risk of the $i$-th spread $Y_{t}^{i}$, i.e. the spread between fuels $i-1$ and $i$. Thus, the assumption of non arbitrage is satisfied in the market of fuels (indeed, one can easily prove that each $\widetilde{Y}^{i}$ is a $\mathbb{Q}^{i}$-martingale if and only if each $\widetilde{S}^{i}$ is a $\widehat{\mathbb{Q}}$-martingale, where ${ }^{-}$denotes discounting $)$. Let $\widehat{W}=\left(\widehat{W}^{1}, \ldots, \widehat{W}^{n}\right)$ be the $\widehat{\mathbb{Q}}$-Brownian motion defined via Girsanov's theorem as:

$$
\widehat{W}_{t}^{i}=W_{t}^{i}+\lambda_{i} t
$$

Finally, one can show that the $\widehat{\mathbb{Q}}$-dynamics of the $i$-th fuel, $1 \leq i \leq n$, is given by:

$$
d S_{t}^{i}=\frac{1}{h_{i}} \sum_{j \leq i} Y_{t}^{j}\left(r d t+\sigma_{j} d \widehat{W}_{t}^{j}\right)=r S_{t}^{i} d t+\frac{1}{h_{i}} \sum_{j \leq i} Y_{t}^{j} \sigma_{j} d \widehat{W}_{t}^{j}
$$

and that of its forward price $F_{t}^{i}(T)$ with any maturity $T>0$ is:

$$
d F_{t}^{i}(T)=e^{r(T-t)} \frac{1}{h_{i}} \sum_{j \leq i} Y_{t}^{j} \sigma_{j} d \widehat{W}_{t}^{j}
$$

In order to generate the whole family of EMMs, we need to consider also possible changes of measure for the demand $D$ (equivalently its driving Brownian motion $W^{D}$ ) and all the capacities $C^{i}$ (equivalently their driving multivariate Brownian motion $W^{C, i}$ ). Thanks to the mutual independence between fuels, capacities and electricity demand, it is not difficult to obtain the following result. Let $T>0$ be a given finite horizon, e.g. the maturity of a forward contract on electricity. The next proposition gives a full characterization of the (square-integrable) EMMs of our model.

Proposition 3.3. The set of all EMMs $\mathcal{M}_{e}$ of our model over the time horizon $[0, T]$ is given by all $\mathcal{F}_{T}$-measurable random variables $Z_{T}$ such that there exists adapted processes $\eta=\left(\eta^{C}, \eta^{D}\right)$ verifying:

$$
Z_{T}=\left(\prod_{i=1}^{n} \frac{d \mathbb{Q}^{i}}{d \mathbb{P}}\right) \mathcal{E}_{T}\left(\int_{0}^{\cdot} \eta_{s}^{C} d W_{s}^{C}\right) \mathcal{E}_{T}\left(\int_{0}^{\cdot} \eta_{s}^{D} d W_{s}^{D}\right)
$$

and such that $\mathbb{E}_{\mathbb{P}}\left[Z_{T}\right]=1$, where $\mathcal{E}_{T}(\cdot)$ denotes the stochastic exponential at time $T$ and $\mathbb{Q}^{i}$ is the unique EMM for the $i$-th spread $Y^{i}$, for $i=1, \ldots, n$, as in (3.6).

Proof. Use the mutual independence of $\left(W, W^{C}, W^{D}\right)$ and the representation theorem for Brownian martingales.

In view of (3.5) and (3.9), it is easy to see that in our model the Föllmer and Schweizer minimal EMM corresponds to the case when $\eta^{C} \equiv \eta^{D} \equiv 0$ in (3.9). ${ }^{2}$ Thus, consistently with our notation, the minimal EMM is exactly the measure $\widehat{\mathbb{Q}}$ previously introduced.

\footnotetext{
${ }^{2}$ Indeed, in our model, the Radon-Nikodym derivative $\widehat{Z}_{t}=\mathbb{E}\left[d \widehat{Q} / d \mathbb{P} \mid \mathcal{F}_{t}\right]$ clearly satisfies the so-called integrability condition $R_{2}(\mathbb{P})$, so that Theorem C in Delbaen et al. [1997] can be applied.
} 


\subsection{Electricity futures}

As a first important application of the LRM approach, we derive the price dynamics of a forward contract on electricity with instantaneous delivery at a given time $T$ under our model (2.2). Such a dynamics will be of a great importance to obtain hedging strategies for energy derivatives. To do so, we will apply LRM approach for pricing and hedging a futures contract on electricity via trading on fuels. Then, from these results, we will be able to study the risk premium of electricity. Forward contracts on electricity will be used as additional hedging instruments in the next subsections on hedging and pricing of more complex energy derivatives.

\subsubsection{Price and dynamics}

We now derive the price and dynamics of electricity forward contracts. Using the notation of the previous section, the contingent claim to hedge has terminal payoff $H=P_{T}$ and the hedge is performed by trading in the fuel process $S$, i.e. $X=S$, or equivalently in the fuel futures. ${ }^{3}$ Recall from Section 3.1 that we assume a constant spot interest rate $r$ (futures and forwards are thus identical, and we use both terms interchangeably), as well as independence between fuels, demand and capacities. In this setting, and recalling our spot model (2.2), it is not difficult to see that the formula relating the electricity forward price $F_{t}^{e}(T)$ and the forward prices of fuels $F_{t}^{i}(T)$, both with instantaneous delivery period, is given by:

$$
F_{t}^{e}(T)=\sum_{i=1}^{n} h_{i} F_{t}^{i}(T) \mathbb{E}\left[g\left(C_{T}^{\max }-D_{T}\right) \mathbf{1}_{\left\{D_{T} \in I_{T}^{i}\right\}} \mid \mathcal{F}_{t}^{D, C}\right]
$$

for $t \in[0, T]$, where $\mathcal{F}_{t}^{D, C}:=\mathcal{F}_{t}^{D} \vee \mathcal{F}_{t}^{C}$ is the natural filtration generated by both $W^{D}$ and $W^{C}$. Recall that, by the definition of minimal EMM, one has $\widehat{\mathbb{Q}}=\mathbb{P}$ on $\mathcal{F}^{D, C}$.

Remark 3.4. The previous formula is key in our approach. A similar formula is obtained in the previous paper [Aïd et al., 2009] for a slightly different model, the arguments used to prove it being the same. The idea behind it is that, unlike usual approaches in energy market models, in our model the use of a risk-neutral measure is motivated by embedding the energy market into the larger market including the possibility of trading in fuels. Then, since in the latter market one may in principle trade on fuels, the risk-neutral approach that proved to be successful in stock markets can be applied. The final step is that, since a forward contract on energy can be viewed as an option whose payoff is exactly the spot price at maturity and the latter is a function of fuels (via the relation (2.2)), such a forward contract can be priced taking expectation under a risk-neutral measure for fuels. For more details, we refer to Aïd et al. [2009]. The price to pay is, in some sense, that the production function linking fuels and energy contains other non-tradable factors as well, e.g. demand and capacities.

Remark 3.5. The same kind of factorization as in formula (3.4) can be obtained under any EMM $\mathbb{Q}$ such that fuels $S$ are independent from demand and capacities $(D, C)$. For instance, when $\mathbb{Q}$ is an EMM with deterministic (time-dependent) market prices of fuel, demand and capacity risks, i.e. deterministic $\eta^{D}, \eta^{C}$ as in Proposition 3.3, the same computations lead to the same formula. Now, if one takes an arbitrary EMM $\mathbb{Q}$ (under which fuels, demand and capacities are not necessarily independent), one may obtain an analogue formula for electricity futures price via an additional probability change. Indeed, set $d \widehat{\mathbb{Q}}^{i} / d \widehat{\mathbb{Q}}=e^{-r T} S_{T}^{i} / S_{0}^{i}$ on $\mathcal{F}_{T}$ for all $i=1, \ldots, n$. Thus we get:

$$
F_{t}^{e}(T)=\widehat{\mathbb{E}}\left[P_{T} \mid \mathcal{F}_{t}\right]=\sum_{i=1}^{n} h_{i} F_{t}^{i}(T) \widehat{\mathbb{E}}^{i}\left[g\left(C_{T}^{\max }-D_{T}\right) \mathbf{1}_{\left\{D_{T} \in I_{T}^{i}\right\}} \mid \mathcal{F}_{t}^{D, C}\right]
$$

where $\widehat{\mathbb{E}}^{i}$ denotes expectation under $\widehat{\mathbb{Q}}^{i}$. Of course, here the difficulty would be to compute the weights multiplying the futures on fuels. The laws of $D$ and $C$ under each $\widehat{\mathbb{Q}}^{i}$ can of course be obtained through Girsanov's theorem, but their parameters might depend on $S, C$ and $D$ in a complicated way, and obtaining closed-form formulae may be very difficult.

\footnotetext{
${ }^{3}$ Indeed, one may easily switch from $F_{t}^{i}(T)$ to $S_{t}^{i}$ via the well-known formula $F_{t}^{i}(T)=e^{r(T-t)} S_{t}^{i}$. Recall that we assumed constant interest rate $r$ and zero convenience yield and storage cost, for any fuel $i$.
} 
The next step consists in evaluating the conditional expectation appearing in formula (3.4). Since the process $(C, D)$ is Markov, we have:

$$
\mathbb{E}\left[g\left(C_{T}^{\max }-D_{T}\right) \mathbf{1}_{\left\{D_{T} \in I_{T}^{i}\right\}} \mid \mathcal{F}_{t}^{D, C}\right]=G_{i}^{T}\left(t, C_{t}, D_{t}\right)
$$

for some real-valued measurable function $G_{i}^{T}(t, c, d)$ defined on $[0, T] \times \mathbb{R}^{n} \times \mathbb{R}$. We will call this function the Conditional Expectation of Scarcity function (henceforth CES function). Under specific dynamics for $C_{t}$ and $D_{t}$, the CES function, as well as its partial derivatives, can be computed explicitly. This will be the purpose of Section 4.2. Our key relation (3.4) between electricity forwards and fuel forwards now simply reads:

$$
F_{t}^{e}(T)=\sum_{i=1}^{n} h_{i} G_{i}^{T}\left(t, C_{t}, D_{t}\right) F_{t}^{i}(T)
$$

meaning that an electricity forward can be seen as a basket of fuels forwards, with stochastic weights given by the CES function, driven by electricity demand and production capacities. Note that this relation does not depend on the specific model chosen for fuels in Section 3.1, except for the assumption that fuels are independent of capacities and demand.

We now derive the dynamics of electricity forwards. Assume that $G_{i}^{T} \in \mathcal{C}^{1,2,2}\left([0, T] \times \mathbb{R}^{n} \times \mathbb{R}\right)^{4}$. Then Itō's lemma provides the dynamics of $G_{i}^{T}\left(t, C_{t}, D_{t}\right)$ as follows:

$$
d G_{i}^{T}\left(t, C_{t}, D_{t}\right)=\sum_{k=1}^{n} \frac{\partial G_{i}^{T}}{\partial c_{k}}\left(t, C_{t}, D_{t}\right) \beta_{k}\left(t, C_{t}^{k}\right) d W_{t}^{C, k}+\frac{\partial G_{i}^{T}}{\partial d}\left(t, C_{t}, D_{t}\right) b\left(t, D_{t}\right) d W_{t}^{D}
$$

A simple application of integration-by-parts formula gives the following $\widehat{\mathbb{Q}}$-dynamics for electricity $T$-forward prices :

$$
\begin{aligned}
d F_{t}^{e}(T)= & \sum_{i=1}^{n} h_{i}\left[G_{i}^{T}\left(t, C_{t}, D_{t}\right) d F_{t}^{i}(T)+F_{t}^{i}(T) d G_{i}^{T}\left(t, C_{t}, D_{t}\right)\right] \\
= & e^{r(T-t)} \sum_{i=1}^{n}\left(\sum_{k=i}^{n} G_{k}^{T}\left(t, C_{t}, D_{t}\right)\right) \sigma_{i} Y_{t}^{i} d \widehat{W}_{t}^{i}+\sum_{i=1}^{n} h_{i} F_{t}^{i}(T) \frac{\partial G_{i}^{T}}{\partial d}\left(t, C_{t}, D_{t}\right) b\left(t, D_{t}\right) d W_{t}^{D} \\
& +\sum_{i=1}^{n} h_{i} F_{t}^{i}(T) \sum_{k=1}^{n} \frac{\partial G_{i}^{T}}{\partial c_{k}}\left(t, C_{t}, D_{t}\right) \beta_{k}\left(t, C_{t}^{k}\right) d W_{t}^{C, k}
\end{aligned}
$$

where recall from Section 3.2.2 that $\widehat{W}_{t}^{i}$ is a $\widehat{\mathbb{Q}}$-Brownian motion. Notice that the quadratic covariation between $F_{t}^{i}(T)$ and $G_{i}\left(t, C_{t}, D_{t}\right)$ vanishes, due once more to the independence between $S^{i}$ and $(C, D)$. From equation (3.7), one can deduce the $\mathbb{P}$-dynamics of $F^{e}(T)$ :

$$
\begin{aligned}
d F_{t}^{e}(T)= & e^{r(T-t)} \sum_{i=1}^{n}\left(\sum_{k=i}^{n} G_{k}^{T}\left(t, C_{t}, D_{t}\right)\right) \sigma_{i} Y_{t}^{i}\left(d W_{t}^{i}+\lambda_{i} d t\right) \\
& +\sum_{i=1}^{n} h_{i} F_{t}^{i}(T) \frac{\partial G_{i}^{T}}{\partial d}\left(t, C_{t}, D_{t}\right) b\left(t, D_{t}\right) d W_{t}^{D} \\
& +\sum_{i=1}^{n} h_{i} F_{t}^{i}(T) \sum_{k=1}^{n} \frac{\partial G_{i}^{T}}{\partial c_{k}}\left(t, C_{t}, D_{t}\right) \beta_{k}\left(t, C_{t}^{k}\right) d W_{t}^{C, k}
\end{aligned}
$$

\subsubsection{Risk premium}

Using the previous results, we are able to study the electricity risk premium $\pi^{e}(t, T)$, defined as:

$$
\pi^{e}(t, T):=F_{t}^{e}(T)-\mathbb{E}\left[P_{T} \mid \mathcal{F}_{t}\right], \quad t \leq T .
$$

\footnotetext{
${ }^{4}$ Whether such an assumption is verified or not will depend on the regularity of the coefficients of the SDEs governing the dynamics of $D$ and $C$. Notice that the dynamics that we will use in Section 4 are such that the CES functions $G_{i}^{T}(t, c, z)$ are smooth enough to apply Itō's formula (see also the explicit formulae for the derivatives of CES functions in 4.2.7).
} 
Just like the relation (3.11) between futures prices, the electricity risk premium can be expressed as a weighted linear combination of fuel risk premiums. Let $\pi^{i}(t, T)$ denote the risk premium of the $i$-th fuel, i.e. $\pi^{i}(t, T):=F_{t}^{i}(T)-\mathbb{E}_{\mathbb{P}}\left[S_{T}^{i} \mid \mathcal{F}_{t}\right]$ for $t \leq T$. Recall that forward prices are computed under the minimal EMM $\widehat{\mathbb{Q}}$.

Proposition 3.6. Under our model assumptions, one has:

$$
\pi^{e}(t, T)=\sum_{i=1}^{n} h_{i} G_{i}^{T}\left(t, C_{t}, D_{t}\right) \pi^{i}(t, T), \quad t \in[0, T] .
$$

Proof. It follows from formulae (2.2) and (3.11), and the fact that the law of the processes $(C, D)$ under $\widehat{\mathbb{Q}}$ is the same as under $\mathbb{P}$.

Remark 3.7. An easy consequence of the previous equality is that if all the fuels are in normal backwardation (or in contango), then it also holds for electricity. To our knowledge, no studies have been performed to assess this particular point. Nevertheless, in Lautier and Raynaud [2011] a systematic analysis of commodity term structures is done. The authors show that energy prices (crude, heating oil, gasoil, gas) are at least $40 \%$ of the time in contango. Hence, it is not unreasonable to imagine that situations where they are all in contango at the same time may arise.

\subsubsection{Electricity futures as hedging instruments}

In the next sections, we will use these electricity forward contracts as tradable hedging instruments to improve the hedging of more complex derivatives on electricity spot and forwards. In other terms, we will consider an enlarged market $\left(S, F^{e}\left(T^{*}\right)\right)$ where agents can trade on fuels as well as on a forward contract with a given maturity $T^{*}$. While the minimal EMM for the market of fuels $S$ is $\widehat{\mathbb{Q}}$, it is not garanteed a priori that the minimal EMM for the richer market $\left(S, F^{e}\left(T^{*}\right)\right)$ is still $\widehat{\mathbb{Q}}$. It will depend on the $\mathbb{P}$-dynamics of the forward contract $F^{e}\left(T^{*}\right)$. We conclude this part of the paper by showing that if the $\mathbb{P}$-dynamics of $F^{e}\left(T^{*}\right)$ is given exactly by (3.14), then the minimal EMM for $\left(S, F^{e}\left(T^{*}\right)\right)$ is given by $\widehat{\mathbb{Q}}$.

Proposition 3.8. Let $T^{*}$ be any positive finite maturity. Assume that the $\mathbb{P}$-dynamics of $S$ and $F^{e}\left(T^{*}\right)$ are given by, respectively, (3.1) and (3.14). Then, the minimal EMM for $\left(S, F^{e}\left(T^{*}\right)\right)$ is given by $\widehat{\mathbb{Q}}$.

Proof. By the definition of the minimal EMM (see Definition (3.2) in Föllmer and Schweizer [1991]), we have to verify that any square-integrable $\mathbb{P}$-martingale $M$ that is orthogonal to both $S$ and $F^{e}\left(T^{*}\right)$, must be a $\widehat{\mathbb{Q}}$-martingale. By the representation theorem of Brownian martingales and since $\mathcal{F}_{t}^{W}=\mathcal{F}_{t}^{W^{S}}$ for any $t$, such a $\mathbb{P}$-martingale $M$ satisfies

$$
M_{t}=M_{0}+\int_{0}^{t} \alpha_{s} d W_{s}^{S}+\int_{0}^{t} \beta_{s} d W_{s}^{C}+\int_{0}^{t} \gamma_{s} d W_{s}^{D}
$$

for some predictable processes $\alpha, \beta, \gamma . M$ being orthogonal to $S$, i.e. $\left\langle M, W^{S}\right\rangle=0$, we have $\alpha \equiv 0$. Moreover, $M$ is also orthogonal to $F^{e}\left(T^{*}\right)$, which implies that

$$
\left\langle M, F^{e}\left(T^{*}\right)\right\rangle_{t}=\int_{0}^{t} \beta_{s} \theta_{s}^{C} d s+\int_{0}^{t} \gamma_{s} \theta_{s}^{D} d s=0
$$

for all $t$, where $\theta^{C, k}$ (for $k=1, \ldots, n$ ) and $\theta^{D}$ are the integrands in, respectively, the $d W^{C, k}$ part and the $d W^{D}$ part appearing in the dynamics (3.14) of forward prices. Thus, $\beta_{t} \theta_{t}^{C}+\gamma_{t} \theta_{t}^{D} \equiv 0$ for all $t$. As a consequence, since $W^{C}$ and $W^{D}$ are Brownian motions under $\widehat{\mathbb{Q}}, M$ is a $\widehat{\mathbb{Q}}$-martingale. By uniqueness of the minimal EMM, we can conclude. 


\subsection{Pricing formulae}

In this section we compute the price of energy derivatives via the LRM approach. It consists in computing the expectation of the terminal pay-off under the minimal EMM $\widehat{\mathbb{Q}}$. Such an expectation represents the initial wealth allowing to approximately replicate a given option in a local risk minimization sense, as explained in Section 3.2.1.

In what follows, we will focus on options on spreads (between electricity and fuels) as well as on options on electricity forward contracts. We will see in particular that any European options on an electricity forward contract can be viewed as a basket option on fuels with random (but independent) coefficients. Thus, numerical methods developed to price basket options on securities can be applied to evaluate energy options as well. Finally, we will show how to obtain explicit formulae in the case of two fuels, i.e. $n=2$.

We will use the notation $B S_{t}(\sigma, K)$ for the Black-Scholes formula of the $t$-price of a European call option with volatility $\sigma$ and strike $K$. As the other parameters (like maturity or interest rate) are fixed, they will not appear in the notation. Finally, $f_{X}(\cdot)$ (resp. $\hat{f}_{X}(\cdot)$ ) will denote the density at time $T$ of a process $X$ under the statistical measure $\mathbb{P}$ (resp. under the minimal EMM $\widehat{\mathbb{Q}}$ ).

\subsubsection{Spread options}

Let us consider a spread option with maturity $T$ between electricity and a fuel $j$ chosen among the $n$ fuels used to produce electricity. Then, the corresponding pay-off is given by:

$$
H:=\varphi\left(P_{T}-h_{j} S_{T}^{j}\right)
$$

where $\varphi$ is a real-valued function such that $H \in L^{2}(\widehat{\mathbb{Q}})$, e.g. $\varphi(x)=(x-K)^{+}$for $K \geq 0$. For the sake of simplicity, we compute the price of this option at time $t=0$. The price at any time $t$ can be easily deduced from that case by using the Markov property of the price processes. Using equation (2.2), one obtains :

$$
\pi_{0}:=e^{-r T} \widehat{\mathbb{E}}\left[\varphi\left(P_{T}-h_{j} S_{T}^{j}\right)\right]=e^{-r T} \sum_{i=1}^{n} \widehat{\mathbb{E}}\left[\varphi\left(g\left(C_{T}^{\max }-D_{T}\right) h_{i} S_{T}^{i}-h_{j} S_{T}^{j}\right) \mathbf{1}_{\left\{D_{T} \in I_{T}^{i}\right\}}\right] .
$$

Now, consider the case of two fuels $(n=2)$ and let $j=1$. The other case $j=2$ can be treated similarly. Recall from equation (2.1) that in the two fuels case, the intervals $I_{T}^{i}$ are $I_{T}^{1}=\left(-\infty, C_{T}^{1}\right)$ and $I_{T}^{2}=\left[C_{T}^{1},+\infty\right)$. Using the independence between fuels $S$, demand $D$ and capacities $C$, and the fact that $\widehat{\mathbb{Q}}=\mathbb{P}$ on $\mathcal{F}_{T}^{C, D}$, we have:

$$
\pi_{0}=\int_{\mathbb{R}^{2}} f_{C_{T}^{1}-D_{T}}(z) f_{C_{T}^{2}}(c)\left\{\phi_{1}(c, z) \mathbf{1}_{\{z>0\}}+\phi_{2}(c, z) \mathbf{1}_{\{z \leq 0\}}\right\} d c d z
$$

where $f_{C_{T}^{1}-D_{T}}($.$) and f_{C_{T}^{2}}($.$) are the \mathbb{P}$-densities of, respectively, $C_{T}^{1}-D_{T}$ and $C_{T}^{2}$, while $\phi_{1}(c, z)$ and $\phi_{2}(c, z)$ are given by:

$$
\begin{array}{rll}
\phi_{1}(c, z) & :=e^{-r T} & \widehat{\mathbb{E}}\left[\varphi\left((g(c+z)-1) Y_{T}^{1}\right)\right] \\
\phi_{2}(c, z) & :=e^{-r T} & \widehat{\mathbb{E}}\left[\varphi\left(g(c+z) h_{2} S_{T}^{2}-h_{1} S_{T}^{1}\right)\right] \\
& =e^{-r T} & \widehat{\mathbb{E}}\left[\varphi\left((g(c+z)-1) Y_{T}^{1}+g(c+z) Y_{T}^{2}\right)\right] .
\end{array}
$$

We used the fact that $h_{1} S^{1}=Y^{1}$ and $h_{2} S^{2}=Y^{1}+Y^{2}$ (see Section 3.1). Recall that, by assumption, $Y^{1}$ and $Y^{2}$ are independent geometric Brownian motions. We need to compute both terms $\phi_{i}(c, z)$. Since $c, z$ are fixed, we can simplify the notation by dropping $(c, z)$ in $\phi_{i}(c, z)(i=1,2)$ and $g(c+z)$. We will simply write $\phi_{i}$ and $g$ instead.

To push further our computations, let us consider a call spread option, i.e. $\varphi(x)=(x-K)^{+}$for a given strike $K>0$. In this case, the quantities $\phi_{i}$ can be computed more explicitly. Indeed, if $g \leq 1$ one has $\phi_{1}=0$, while on the event $\{g>1\}$ the quantity $\phi_{1}$ is just $(g-1)$ times the Black-Scholes 
formula for a European call option with strike $K /(g-1)$ and underlying $Y^{1}$, a geometric Brownian motions with volatility $\sigma_{1}$, i.e.

$$
\phi_{1}=(g-1) B S_{0}\left(\sigma_{1}, \frac{K}{g-1}\right) \mathbf{1}_{\{g>1\}} .
$$

On the other hand, we show that $\phi_{2}$ is a mixture of Black-Scholes formulae with respect to strikes. Recall that $\hat{f}_{Y_{T}^{i}}\left(y_{i}\right)$ denotes the $\log$-normal density of $Y_{T}^{i}$ under $\widehat{\mathbb{Q}}$ for $i=1,2$. We have:

$$
\phi_{2}=e^{-r T \widehat{\mathbb{E}}}\left[\left((g-1) Y_{T}^{1}+g Y_{T}^{2}-K\right)^{+}\right]
$$

On the event $\{g \leq 1\}$, we have:

$$
\phi_{2}=g e^{-r T \widehat{\mathbb{E}}}\left[\left(Y_{T}^{2}-\frac{K+(1-g) Y_{T}^{1}}{g}\right)^{+}\right]=g \int_{0}^{\infty} \hat{f}_{Y_{T}^{1}}(y) B S_{0}\left(\sigma_{2}, \frac{K+(1-g) y}{g}\right) d y .
$$

On the opposite event $\{g>1\}$, we have:

$$
\begin{aligned}
& \phi_{2}=g e^{-r T \widehat{\mathbb{E}}}\left[\left(Y_{T}^{2}-\frac{K-(g-1) Y_{T}^{1}}{g}\right)^{+}\right] \\
& =g \int_{0}^{\infty} \hat{f}_{Y_{T}^{1}}(y) e^{-r T \widehat{\mathbb{E}}}\left[\left(Y_{T}^{2}-\frac{K-(g-1) y}{g}\right)^{+}\right] d y \\
& =g \int_{0}^{\frac{K}{g-1}} \hat{f}_{Y_{T}^{1}}(y) B S_{0}\left(\sigma_{2}, \frac{K-(g-1) y}{g}\right) d y+g \int_{\frac{K}{g-1}}^{\infty} \hat{f}_{Y_{T}^{1}}(y) e^{-r T \widehat{\mathbb{E}}}\left[Y_{T}^{2}-\frac{K-(g-1) y}{g}\right] d y \\
& =g \int_{0}^{\frac{K}{g-1}} \hat{f}_{Y_{T}^{1}}(y) B S_{0}\left(\sigma_{2}, \frac{K-(g-1) y}{g}\right) d y+\left(g Y_{0}^{2}-e^{-r T} K\right) \widehat{\mathbb{Q}}\left(Y_{T}^{1} \geq \frac{K}{g-1}\right) \\
& +(g-1) e^{-r T \widehat{\mathbb{E}}}\left[Y_{T}^{1} \mathbf{1}_{\left\{Y_{T}^{1}>\frac{K}{g-1}\right\}}\right] .
\end{aligned}
$$

Observe that:

$$
e^{-r T \widehat{\mathbb{E}}}\left[Y_{T}^{1} \mathbf{1}_{\left\{Y_{T}^{1}>\frac{K}{g-1}\right\}}\right]=B S_{0}\left(\sigma_{1}, \frac{K}{g-1}\right)+e^{-r T} \frac{K}{g-1} \widehat{\mathbb{Q}}\left[Y_{T}^{1}>\frac{K}{g-1}\right]
$$

so that on $\{g>1\}$ we have:

$$
\phi_{2}=g \int_{0}^{\frac{K}{g-1}} \hat{f}_{Y_{T}^{1}}(y) B S_{0}\left(\sigma_{2}, \frac{K-(g-1) y}{g}\right) d y+g Y_{0}^{2} \widehat{\mathbb{Q}}\left(Y_{T}^{1} \geq \frac{K}{g-1}\right)+(g-1) B S_{0}\left(\sigma_{1}, \frac{K}{g-1}\right) .
$$

Since, from Sections 3.1 and 3.2.2, $Y^{1}$ is a geometric Brownian motion under $\widehat{\mathbb{Q}}$, with volatility $\sigma_{1}$ and drift $r$, we have

$$
\widehat{\mathbb{Q}}\left(Y_{T}^{1} \geq \frac{K}{g-1}\right)=\mathcal{N}\left(\frac{\left(r-\frac{\sigma_{1}^{2}}{2}\right) T-\ln \left(\frac{K}{(g-1) Y_{0}^{1}}\right)}{\sigma_{1} \sqrt{T}}\right),
$$

where $\mathcal{N}$ is the cumulative distribution function of a standard normal random variable. We summarize these results in the following proposition:

Proposition 3.9. Let $n=2$, i.e. electricity is produced out of two fuels. The price $\pi_{0}$ at time $t=0$ of a call spread option with pay-off $H=\left(P_{T}-h_{1} S_{T}^{1}-K\right)^{+}, K>0$, is given by the following formula:

$$
\pi_{0}=\int_{\mathbb{R}^{2}} f_{C_{T}^{1}-D_{T}}(z) f_{C_{T}^{2}}(c)\left\{\phi_{1}(c, z) \mathbf{1}_{\{z>0\}}+\phi_{2}(c, z) \mathbf{1}_{\{z \leq 0\}}\right\} d c d z,
$$


where the quantities $\phi_{i}=\phi_{i}(c, z), i=1,2$, are given by:

$$
\begin{aligned}
\phi_{1}= & (g-1) B S_{0}\left(\sigma_{1}, \frac{K}{g-1}\right) \mathbf{1}_{\{g>1\}} \\
\phi_{2}= & g \int_{0}^{\infty} \hat{f}_{Y_{T}^{1}}(y) B S_{0}\left(\sigma_{2}, \frac{K+(1-g) y}{g}\right)\left(\mathbf{1}_{\{g \leq 1\}}+\mathbf{1}_{\{g>1\}} \mathbf{1}_{\left\{y<\frac{K}{g-1}\right\}}\right) d y \\
& +\left(g Y_{0}^{2} \mathcal{N}\left(\frac{\left(r-\frac{\sigma_{1}^{2}}{2}\right) T-\ln \left(\frac{K}{(g-1) Y_{0}^{1}}\right)}{\sigma_{1} \sqrt{T}}\right)+(g-1) B S_{0}\left(\sigma_{1}, \frac{K}{g-1}\right)\right) \mathbf{1}_{\{g>1\}}
\end{aligned}
$$

where we have set $g:=g(c+z)$.

\subsubsection{Options on electricity forwards}

Let us consider a contingent claim $H$ with maturity $T$ whose payoff is given by a function $\varphi: \mathbb{R} \mapsto \mathbb{R}$ of a forward contract on electricity with instantaneous delivery period at $T^{*}>T$, i.e.

$$
H:=\varphi\left(F_{T}^{e}\left(T^{*}\right)\right)
$$

We assume that $\varphi$ is such that $H \in L^{2}(\widehat{\mathbb{Q}})$. The next proposition gives a pricing formula for such a contingent claim.

Proposition 3.10. Under the above assumptions, the price at time $t \leq T$ of the contingent claim $H$ is given by:

$$
\widehat{\mathbb{E}}\left[e^{-r(T-t)} H \mid \mathcal{F}_{t}\right]=\widehat{\mathbb{E}}\left[\psi\left(t, F_{t}\left(T^{*}\right), C_{T}, D_{T}\right) \mid \mathcal{F}_{t}^{D, C}\right]
$$

where:

$$
\psi\left(t, F_{t}\left(T^{*}\right), C_{T}, D_{T}\right):=e^{-r(T-t)} \widehat{\mathbb{E}}\left[\varphi\left(\sum_{i=1}^{n} h_{i} G_{i}^{T^{*}}\left(T, C_{T}, D_{T}\right) F_{T}^{i}\left(T^{*}\right)\right) \mid \mathcal{F}_{t}^{W}\right] .
$$

Proof. It follows from equation (3.11), independence between $W$ and $\left(W^{D}, W^{C}\right)$ and the properties of conditional expectations.

Remark 3.11. The previous pricing formula (3.20) provides an easy way of computing prices using basket options pricing algorithms. Indeed, the formula suggests the following procedure:

1. Evaluate first the expectation (3.21) with respect to $\mathcal{F}_{t}^{W}$, i.e. the function $\psi$, taking the weights $G_{i}^{T^{*}}\left(T, C_{T}, D_{T}\right)$ as fixed, using a basket options evaluation procedure.

2. Finally, take the average with respect to the weights $G_{i}^{T^{*}}\left(T, C_{T}, D_{T}\right)$.

These two steps can be performed separately thanks to independence between $W$ and $\left(W^{D}, W^{C}\right)$.

As for spread options, we now look for explicit formulae for European call on electricity forward in the two fuels case, i.e. $n=2$, at time $t=0$. We compute the function $\psi$ from equation (3.21) in this case. To simplify notation, we set $w_{i}:=e^{-r\left(T^{*}-T\right)} G_{i}^{T^{*}}\left(T, C_{T}, D_{T}\right)$. Recalling that, regarding futures on fuels, $F_{T}^{i}\left(T^{*}\right)=e^{r\left(T^{*}-T\right)} S_{T}^{i}$ for $i=1$, 2, we have:

$$
\begin{aligned}
\psi(0)= & e^{-r T \widehat{\mathbb{E}}}\left[\left(w_{1} h_{1} S_{T}^{1}+w_{2} h_{2} S_{T}^{2}-K\right)^{+}\right]=e^{-r T \widehat{\mathbb{E}}}\left[\left(\left(w_{1}+w_{2}\right) Y_{T}^{1}-\left(K-w_{2} Y_{T}^{2}\right)\right)^{+}\right] \\
= & e^{-r T \widehat{\mathbb{E}}}\left[\left(\left(w_{1}+w_{2}\right) Y_{T}^{1}-\left(K-w_{2} Y_{T}^{2}\right)\right)^{+} \mathbf{1}_{\left\{Y_{T}^{2} \leq K / w_{2}\right\}}\right] \\
& +e^{-r T \widehat{\mathbb{E}}}\left[\left(\left(w_{1}+w_{2}\right) Y_{T}^{1}-\left(K-w_{2} Y_{T}^{2}\right)\right)^{+} \mathbf{1}_{\left\{Y_{T}^{2}>K / w_{2}\right\}}\right]=: A_{1}+A_{2} .
\end{aligned}
$$

Let us compute separately $A_{1}$ and $A_{2}$. For $A_{1}$, we obtain:

$$
\begin{aligned}
A_{1} & =\left(w_{1}+w_{2}\right) \int_{0}^{K / w_{2}} \hat{f}_{Y_{T}^{2}}(y) \widehat{\mathbb{E}}\left[e^{-r T}\left(Y_{T}^{1}-\frac{K-w_{2} y}{w_{1}+w_{2}}\right)^{+}\right] d y \\
& =\left(w_{1}+w_{2}\right) \int_{0}^{K / w_{2}} \hat{f}_{Y_{T}^{2}}(y) B S_{0}\left(\sigma_{1}, \frac{K-w_{2} y}{w_{1}+w_{2}}\right) d y .
\end{aligned}
$$


For $A_{2}$, we have:

$$
\begin{aligned}
A_{2} & =e^{-r T \widehat{\mathbb{E}}}\left[\left(\left(w_{1}+w_{2}\right) Y_{T}^{1}-\left(K-w_{2} Y_{T}^{2}\right)\right) \mathbf{1}_{\left\{Y_{T}^{2}>K / w_{2}\right\}}\right] \\
& =\left(w_{1}+w_{2}\right) \widehat{\mathbb{E}}\left[e^{-r T} Y_{T}^{1} \mathbf{1}_{\left\{Y_{T}^{2}>K / w_{2}\right\}}\right]+w_{2} \widehat{\mathbb{E}}\left[e^{-r T}\left(Y_{T}^{2}-\frac{K}{w_{2}}\right) \mathbf{1}_{\left\{Y_{T}^{2}>K / w_{2}\right\}}\right] \\
& =\left(w_{1}+w_{2}\right) Y_{0}^{1} \widehat{\mathbb{Q}}\left(Y_{T}^{2}>K / w_{2}\right)+w_{2} B S_{0}\left(\sigma_{2}, \frac{K}{w_{2}}\right)
\end{aligned}
$$

We summarize our findings in the following proposition.

Proposition 3.12. Consider the two fuels case, i.e. $n=2$. The price $\pi_{0}^{F}$ at time $t=0$ of a European call option with maturity $T$ on a $T^{*}$-forward contract on electricity with $T^{*}>T$ is given by the following formula:

$$
\pi_{0}^{F}=\int_{\mathbb{R}} f_{D_{T}}(z) \int_{\mathbb{R}^{2}} f_{C_{T}^{1}}\left(c_{1}\right) f_{C_{T}^{2}}\left(c_{2}\right) \psi_{0}\left(c_{1}, c_{2}, z\right) d z d c_{1} d c_{2},
$$

where the function $\psi_{0}\left(c_{1}, c_{2}, z\right)$ is given by:

$$
\begin{aligned}
\psi_{0}\left(c_{1}, c_{2}, z\right)= & \left(w_{1}+w_{2}\right)\left\{\int_{0}^{K / w_{2}} \hat{f}_{Y_{T}^{2}}(y) B S_{0}\left(\sigma_{1}, \frac{K-w_{2} y}{w_{1}+w_{2}}\right) d y+Y_{0}^{1} \mathcal{N}\left(\frac{\left(r-\frac{\sigma_{2}^{2}}{2}\right) T-\ln \left(\frac{K}{w_{2}}\right)}{\sigma_{2} \sqrt{T}}\right)\right\} \\
& +w_{2} B S_{0}\left(\sigma_{2}, \frac{K}{w_{2}}\right)
\end{aligned}
$$

where $w_{i}:=e^{-r\left(T^{*}-T\right)} G_{i}^{T^{*}}\left(T, c_{1}, c_{2}, z\right), i=1,2$.

To make full use of this result, the weights $w_{i}$, and thus the CES function $G_{i}^{T^{*}}\left(T, c_{1}, c_{2}, z\right)$, must be computed explicitly. This will be done in Section 4.2 under more specific assumptions on dynamics of capacities $C$ and demand $D$.

\subsection{Hedging derivatives}

Now we turn to hedging. In this subsection, we will identify the hedgeable and the unhedgeable part of any contingent claim written on electricity as well as fuels.

As hedging instruments, we consider forward contracts on electricity and forward contracts on fuels (or, equivalently, spot fuels). We consider a $\widehat{\mathbb{Q}}$-square integrable European-type contingent claim $H$ written on a forward contract on electricity and fuels as well as on capacities and energy demand, i.e.

$$
H=\varphi\left(F_{T}^{e}\left(T^{*}\right), F_{T}\left(T^{*}\right), C_{T}, D_{T}\right)
$$

such that $H \in L^{2}(\widehat{\mathbb{Q}})$. Notice that spread options (Section 3.4.1) and options on electricity forwards (Section 3.4.2) are of this type, as well as any option on electricity spot price.

Since $\widehat{\mathbb{Q}}$ is the minimal EMM for the market of fuels $S$ (see Section 3.2.2) as well as for the larger market $\left(S, F^{e}\left(T^{*}\right)\right)$ of fuels and electricity $T^{*}$-forward contract (see Proposition 3.8 ), we have to find, according to Theorem 3.2, an explicit expression for the GKW decomposition of such an $H$ under $\widehat{\mathbb{Q}}$. More precisely, being $F^{e}\left(T^{*}\right)$ and $F^{i}\left(T^{*}\right), 1 \leq i \leq n$ our hedging instruments, so we look for self-financing strategies $\xi^{e}$ and $\xi=\left(\xi^{1}, \ldots, \xi^{n}\right)$ such that:

$$
H=\widehat{\mathbb{E}}[H]+\int_{0}^{T} \xi_{t} \cdot d F_{t}\left(T^{*}\right)+\int_{0}^{T} \xi_{t}^{e} d F_{t}^{e}\left(T^{*}\right)+L_{T}^{H}
$$

where $L_{T}^{H}$ is the terminal value of a $\widehat{\mathbb{Q}}$-martingale $L^{H}$ orthogonal to $F^{e}\left(T^{*}\right)$ and $F\left(T^{*}\right)$, representing the unhedgeable risk related to the contingent claim $H$. We are going to explicitly compute such strategies as well as the unhedgeable risk $L_{T}^{H}$. We adapt methods developed in, e.g., Heath et al. [2001], for stochastic volatility diffusion models. 
First, let $\theta=\left(\theta^{S}, \theta^{C}, \theta^{D}\right)$ denote the integrands in the $\widehat{\mathbb{Q}}$-dynamics for the forward price $F^{e}\left(T^{*}\right)$ given by equation (3.13), i.e.:

$$
d F_{t}^{e}=\theta_{t}^{S} \cdot d \widehat{W}_{t}+\theta_{t}^{C} \cdot d W_{t}^{C}+\theta_{t}^{D} d W_{t}^{D}
$$

By the Markov property of the vector-valued process $\left(F\left(T^{*}\right), C, D\right)$ and by the fact that $F_{t}^{e}\left(T^{*}\right)$ is a function of $\left(t, F_{t}\left(T^{*}\right), C_{t}, D_{t}\right)$ (see formula $\left.(3.11)\right)$, we have

$$
V_{t}^{H}:=\widehat{\mathbb{E}}\left[H \mid \mathcal{F}_{t}\right]=\phi\left(t, F_{t}\left(T^{*}\right), C_{t}, D_{t}\right)
$$

for some measurable function $\phi:[0, T] \times \mathbb{R} \times \mathbb{R}^{n} \times \mathbb{R}^{n} \times \mathbb{R} \mapsto \mathbb{R}$. Moreover, under some regularity assumptions on the coefficients of the underlying processes, which are satisfied by the model considered in, e.g., Section 4 , such a function is of class $\mathcal{C}^{1,2,2,2}\left([0, T] \times \mathbb{R}^{n} \times \mathbb{R}^{n} \times \mathbb{R}\right)^{5}$. From now on we drop, for the sake of simplicity, the dependence from $\left(t, F_{t}, C_{t}, D_{t}\right)$ from the function $\phi$ and its derivatives. In the next proposition we will use the notation $\left\|\theta_{t}^{C}, \theta_{t}^{D}\right\|$ for the norm of the vector $\left(\theta_{t}^{C}, \theta_{t}^{D}\right)$.

Proposition 3.13. Let $H=\varphi\left(F_{T}^{e}\left(T^{*}\right), F_{T}\left(T^{*}\right), C_{T}, D_{T}\right) \in L^{2}(\widehat{\mathbb{Q}})$ be a $T$-contingent claim with $T \leq T^{*}$. The local risk minimizing strategy $\left(\xi^{e}, \xi\right)$ is given by:

$$
\begin{aligned}
\xi_{t}^{e} & =\frac{1}{\left\|\theta_{t}^{C}, \theta_{t}^{D}\right\|^{2}}\left\{\sum_{i=1}^{n} \theta_{t}^{C, i} \frac{\partial \phi}{\partial c_{i}} \beta_{i}\left(t, C_{t}^{i}\right)+\theta_{t}^{D} \frac{\partial \phi}{\partial d} b\left(t, D_{t}\right)\right\} \\
\xi_{t}^{i} & =\frac{\partial \phi}{\partial y_{i}}+\frac{h_{i} G_{i}^{T^{*}}\left(t, C_{t}, D_{t}\right)}{\left\|\theta_{t}^{C}, \theta_{t}^{D}\right\|^{2}}\left\{\sum_{i=1}^{n} \theta_{t}^{C, i} \frac{\partial \phi}{\partial c_{i}} \beta_{i}\left(t, C_{t}^{i}\right)+\theta_{t}^{D} \frac{\partial \phi}{\partial d} b\left(t, D_{t}\right)\right\}
\end{aligned}
$$

while the residual risk $L^{H}$ satisfies:

$$
\begin{aligned}
d L_{t}^{H}= & \sum_{i=1}^{n}\left(\frac{\partial \phi}{\partial c_{i}} \beta_{i}\left(t, C_{t}^{i}\right)-\frac{\sum_{k=1}^{n} \theta_{t}^{C, k} \frac{\partial \phi}{\partial c_{k}} \beta_{k}\left(t, C_{t}^{k}\right)+\theta_{t}^{D} \frac{\partial \phi}{\partial d} b\left(t, D_{t}\right)}{\left\|\theta_{t}^{C}, \theta_{t}^{D}\right\|^{2}} \theta_{t}^{C, i} \mathbf{1}_{\left\{\left\|\theta_{t}^{C}, \theta_{t}^{D}\right\|>0\right\}}\right) d W_{t}^{C, i} \\
& -\left(\frac{\partial \phi}{\partial d} b\left(t, D_{t}\right)-\frac{\theta_{t}^{D} \frac{\partial \phi}{\partial d} b\left(t, D_{t}\right)}{\left\|\theta_{t}^{C}, \theta_{t}^{D}\right\|^{2}} \mathbf{1}_{\left\{\left\|\theta_{t}^{C}, \theta_{t}^{D}\right\|>0\right\}}\right) d W_{t}^{D}
\end{aligned}
$$

Proof. The maturity $T^{*}$ of the forward contracts being fixed, we drop it from the notation, so that we now write $F_{t}^{e}, F_{t}^{i}$ and $G_{i}$ instead of, respectively, $F_{t}^{e}\left(T^{*}\right), F_{t}^{i}\left(T^{*}\right)$ and $G_{i}^{T^{*}}$. We also assume w.l.o.g. that $\widehat{\mathbb{E}}[H]=0$. Since $V_{t}^{H}=\widehat{\mathbb{E}}\left[H \mid \mathcal{F}_{t}\right]=\phi\left(t, F_{t}, C_{t}, D_{t}\right)$ with $\phi$ regular enough to apply Itō's lemma, we have that

$$
V_{t}^{H}=\int_{0}^{t} \sum_{i} \frac{\partial \phi}{\partial y_{i}} d F_{s}^{i}+\int_{0}^{t} \sum_{i} \frac{\partial \phi}{\partial c_{i}} \beta_{i}\left(s, C_{s}^{i}\right) d W_{s}^{C, i}+\int_{0}^{t} \frac{\partial \phi}{\partial d} b\left(s, D_{s}\right) d W_{s}^{D} .
$$

Notice that there is no $d t$-term in the expression above since we already know, by definition, that $V_{t}^{H}$ is a martingale under $\widehat{\mathbb{Q}}$. Now, recall from equation $(3.23)$ that:

$$
d F_{t}^{e}=\theta_{t}^{S} \cdot d \widehat{W}_{t}+\theta_{t}^{C} \cdot d W_{t}^{C}+\theta_{t}^{D} d W_{t}^{D}
$$

where explicit expressions for the integrands $\theta=\left(\theta^{S}, \theta^{C}, \theta^{D}\right)$ are provided by equation (3.13). We consider only the non-redundant part of $F^{e}$, i.e. the part that cannot be hedged using fuels, which is given by:

$$
d F_{t}^{C, D}:=\theta_{t}^{C} d W_{t}^{C}+\theta_{t}^{D} d W_{t}^{D}
$$

This process can be rewritten in terms of a suitable Brownian motion $W^{C, D}$ (use Lévy's characterization theorem to prove that $W^{C, D}$ is a Brownian motion) defined as:

$$
W_{t}^{C, D}:=\int_{0}^{t} \frac{\theta_{s}^{C} \cdot d W_{s}^{C}+\theta_{s}^{D} d W_{s}^{D}}{\left\|\theta_{s}^{C}, \theta_{s}^{D}\right\|}
$$

\footnotetext{
${ }^{5}$ See, e.g., Theorem 5.3 in Friedman [1975] for such regularity assumptions
} 
From equation (3.13), one can check that $\left\|\theta_{t}^{C}, \theta_{t}^{D}\right\|>0$ for all $t$. Therefore:

$$
d F_{t}^{C, D}=\left\|\theta_{t}^{C}, \theta_{t}^{D}\right\| d W_{t}^{C, D}, \quad t \in\left[0, T^{*}\right]
$$

Analogously, it holds that:

$$
\sum_{i} \frac{\partial \phi}{\partial c_{i}} \beta_{i}\left(t, C_{t}^{i}\right) d W_{t}^{C, i}+\frac{\partial \phi}{\partial d} b\left(t, D_{t}\right) d W_{t}^{D}=\zeta_{t} d \bar{W}_{t}^{C, D}
$$

where:

$$
\zeta_{t}^{2}:=\sum_{i}\left(\frac{\partial \phi}{\partial c_{i}} \beta_{i}\left(t, C_{t}^{i}\right)\right)^{2}+\left(\frac{\partial \phi}{\partial d} b\left(t, D_{t}\right)\right)^{2}
$$

and $\bar{W}^{C, D}$ is a standard Brownian motion (use Lévy's criterion once more) defined as:

$$
\bar{W}_{t}^{C, D}:=\int_{0}^{t} \frac{\sum_{i} \frac{\partial \phi}{\partial c_{i}} \beta_{i}\left(s, C_{s}^{i}\right) d W_{s}^{C, i}+\frac{\partial \phi}{\partial d} b\left(s, D_{s}\right) d W_{s}^{D}}{\zeta_{s}} .
$$

We require that $\zeta_{t}>0$ for all $t$, which basically means that the contingent claim $H$ does depend on $C$ and $D$. Observe that $W^{C, D}$ and $\bar{W}^{C, D}$ are correlated Brownian motions under both probabilities $\mathbb{P}$ and $\widehat{\mathbb{Q}}$, with quadratic covariation $\rho_{t}$ given by:

$$
\rho_{t}=\frac{\sum_{i} \theta_{t}^{C, i} \frac{\partial \phi}{\partial c_{i}} \beta_{i}\left(t, C_{t}^{i}\right)+\theta_{t}^{D} \frac{\partial \phi}{\partial d} b\left(t, D_{t}\right)}{\left\|\theta_{t}^{C}, \theta_{t}^{D}\right\| \zeta_{t}},
$$

i.e. $d\left\langle W^{C, D}, \bar{W}^{C, D}\right\rangle_{t}=\rho_{t} d t$. We can define a new standard Brownian motion $W^{\perp}$ (under both $\mathbb{P}$ and $\widehat{\mathbb{Q}})$, independent of $W^{C, D}$, such that:

$$
\bar{W}_{t}^{C, D}=\int_{0}^{t} \rho_{s} d W_{s}^{C, D}+\int_{0}^{t} \sqrt{1-\rho_{s}^{2}} d W_{s}^{\perp},
$$

i.e. $W_{t}^{\perp}=\int_{0}^{t} \frac{d \bar{W}_{s}^{C, D}-\rho_{s} d W_{s}^{C, D}}{\sqrt{1-\rho_{s}^{2}}}$. Therefore, we can write:

$$
\sum_{i} \frac{\partial \phi}{\partial c^{i}} \beta_{i}\left(t, C_{t}^{i}\right) d W_{t}^{C, i}+\frac{\partial \phi}{\partial d} b\left(t, D_{t}\right) d W_{t}^{D}=\zeta_{t}\left(\rho_{t} d W_{t}^{C, D}+\sqrt{1-\rho_{t}^{2}} d W_{t}^{\perp}\right)
$$

Finally, since $d W_{t}^{C, D}=\frac{d F_{t}^{e}-\theta_{t}^{S} d \widehat{W}_{t}}{\left\|\theta_{t}^{C}, \theta_{t}^{D}\right\|}$, we have:

$$
\sum_{i} \frac{\partial \phi}{\partial c_{i}} \beta_{i}\left(t, C_{t}^{i}\right) d W_{t}^{C, i}+\frac{\partial \phi}{\partial d} b\left(t, D_{t}\right) d W_{t}^{D}=\zeta_{t}\left(\frac{\rho_{t}}{\left\|\theta_{t}^{C}, \theta_{t}^{D}\right\|}\left(d F_{t}^{e}-\theta_{t}^{S} d \widehat{W}_{t}\right)+\sqrt{1-\rho_{t}^{2}} d W_{t}^{\perp}\right)
$$

and, using the fact that $\theta_{t}^{S, i} d \widehat{W}_{t}^{i}=h_{i} G_{i}\left(t, C_{t}, D_{t}\right) d F_{t}^{i}$ for all $i$, we also have:

$$
V_{t}^{H}=\int_{0}^{t} \frac{\zeta_{s} \rho_{s}}{\left\|\theta_{s}^{C}, \theta_{s}^{D}\right\|} d F_{s}^{e}+\int_{0}^{t} \sum_{i}\left(\frac{\partial \phi}{\partial y_{i}}-\frac{\zeta_{s} \rho_{s}}{\left\|\theta_{s}^{C}, \theta_{s}^{D}\right\|} h_{i} G_{i}\left(s, C_{s}, D_{s}\right)\right) d F_{s}^{i}+\int_{0}^{t} \zeta_{s} \sqrt{1-\rho_{s}^{2}} d W_{s}^{\perp},
$$

which implies that:

$$
\xi_{t}^{e}=\frac{\zeta_{t} \rho_{t}}{\left\|\theta_{t}^{C}, \theta_{t}^{D}\right\|}, \quad \xi_{t}^{i}=\frac{\partial \phi}{\partial y_{i}}+\frac{\zeta_{t} \rho_{t}}{\left\|\theta_{t}^{C}, \theta_{t}^{D}\right\|} h_{i} G_{i}\left(t, C_{t}, D_{t}\right), \quad L_{t}^{H}=\int_{0}^{t} \zeta_{s} \sqrt{1-\rho_{s}^{2}} d W_{s}^{\perp},
$$

are the good candidates for the local risk-minimizing strategy and the residual risk process. Thus, to conclude it suffices to verify that the proposed strategy and residual risk process provide the GKW decomposition under the minimal EMM $\widehat{\mathbb{Q}}$ and apply Theorem 3.2 to get the result. This verification being straightforward, the details are therefore omitted. 
To complete our characterization of the LRM-strategy for $H=\varphi\left(F_{T}^{e}\left(T^{*}\right), F_{T}\left(T^{*}\right), C_{T}, D_{T}\right)$, we have to compute the pricing function $\phi$ and its derivatives, that appear in the formulae for $\xi^{e}$, $\xi$ and $L^{H}$ above. In Section 3.4, we have done so for the pricing function of some specific options. More generally, one can use standard PDE's techniques as follows. Notice that this part is rather formal. Let us consider:

$$
V_{t}^{H}:=\widehat{\mathbb{E}}\left[H \mid \mathcal{F}_{t}\right]=\phi\left(t, F_{t}\left(T^{*}\right), C_{t}, D_{t}\right)
$$

where $F_{t}\left(T^{*}\right)$ denotes the vector $\left(F_{t}^{1}\left(T^{*}\right), \ldots, F_{t}^{n}\left(T^{*}\right)\right)$. Under some regularity assumptions and using Itō's formula, one can prove that the function $\phi(t, y, c, d)$ is the solution to the following PDE:

$$
\begin{aligned}
0= & \frac{\partial \phi}{\partial t}+\sum_{i=1}^{n} \frac{\partial \phi}{\partial c_{i}} \alpha_{i}\left(t, c_{i}\right)+\frac{\partial \phi}{\partial d} a(t, d)+\frac{1}{2} \sum_{i=1}^{n} \frac{\partial^{2} \phi}{\partial y_{i}^{2}}\left[\sum_{j \leq i}\left(y_{j}-y_{j-1}\right)^{2} \sigma_{j}^{2}\right] \\
& +\frac{1}{2} \sum_{i=1}^{n} \frac{\partial^{2} \phi}{\partial c_{i}^{2}} \beta_{i}\left(t, c_{i}\right)^{2}+\frac{1}{2} \frac{\partial^{2} \phi}{\partial d^{2}} b(t, d)^{2}
\end{aligned}
$$

with boundary condition:

$$
\phi(T, y, c, d)=\varphi(y, c, d), \quad \text { for all }(y, c, d) \in \mathbb{R}_{+}^{n} \times \mathbb{R} \times \mathbb{R} .
$$

Notice that the hedging formulae from Proposition 3.13 (through $\theta_{t}^{C}$ and $\theta_{t}^{D}$, see equation (3.11)), as well as the previous pricing PDE, contain the derivatives of the CES function $G_{i}$. These derivatives will be computed explicitly in Section 4.2 .

\section{Numerical results}

\subsection{Explicit model for capacities and demand}

So far, we have worked with the general diffusion models from equations (3.2) and (3.3) for the demand and capacities processes. Now, in order to push further the computations, we are going to choose and estimate more specific models. We decide to model the demand and capacities processes as follows:

$$
\begin{aligned}
D_{t} & =f_{D}(t)+Z_{D}(t) \\
C_{t}^{i} & =f_{i}(t)+Z_{i}(t)
\end{aligned}
$$

where $f_{D}$ and $f_{i}$ are deterministic functions, and $Z_{D}$ and $Z_{i}$ are independent Ornstein-Uhlenbeck (henceforth $\mathrm{OU}$ ) processes under $\mathbb{P}$ :

$$
\begin{aligned}
d Z_{D}(t) & =-\alpha_{D} Z_{D}(t) d t+b d W_{t}^{D} \\
d Z_{i}(t) & =-\alpha_{i} Z_{i}(t) d t+\beta_{i} d W_{t}^{i}
\end{aligned}
$$

where $\alpha_{D}, b, \alpha_{i}, \beta_{i}, 1 \leq i \leq n$ are real constants. In other words, we choose the following functions as coefficients in (3.2) and (3.3):

$$
\begin{aligned}
a(t, d) & =\alpha_{D}\left(f_{D}(t)+\frac{f_{D}^{\prime}(t)}{\alpha_{D}}-d\right) & b(t, d) & =b \\
\alpha_{i}(t, c) & =\alpha_{i}\left(f_{i}(t)+\frac{f_{i}^{\prime}(t)}{\alpha_{i}}-c\right) & \beta_{i}(t, d) & =\beta_{i}
\end{aligned}
$$

We will see that this simple choice combines both satisfactory empirical accuracy and tractability. The ideas behind the definition (4.1) are the following:

- We decompose the demand into a deterministic part, that takes into account in a simple way the yearly and weekly seasonalities, and a stochastic part modeling the randomness of the demand. 
- We use the same decomposition for the capacities, except that only a yearly seasonality is considered (as no statistically significant weekly seasonality appears), and in addition the deterministic part takes into account also the evolution of the installed capacity on the reference fleet. Indeed, should some plants be planned to be added or removed in the near future, the resulting shift in capacity has to be considered.

In equation (4.1), the deterministic functions $f_{D}$ and $f_{i}$ are defined as follows:

$$
\begin{aligned}
f_{D}(t) & =d_{1}+d_{2} \cos \left(2 \pi \frac{t-d_{3}}{l_{1}}\right)+d_{4} \cos \left(2 \pi \frac{t-d_{5}}{l_{2}}\right) \\
f_{i}(t) & =c_{1}^{i}+c_{2}^{i} \cos \left(2 \pi \frac{t-c_{3}^{i}}{l_{1}}\right)+f_{i}^{\text {evo }}(t)
\end{aligned}
$$

where $d_{j}, 1 \leq j \leq 5$ and $c_{k}^{i}, 1 \leq k \leq 3,1 \leq i \leq n$ are constants, and, assuming that $t$ is expressed in years, $l_{1}=1$ (yearly seasonality) and $l_{2} \simeq \frac{1}{52}$ (weekly seasonality), and $f_{i}^{\text {evo }}, 1 \leq i \leq n$ are the deterministic installed capacities evolutions.

Model Estimation. Working on the dataset described in Section 2.2.1, we first estimate, using non-linear least squares, the deterministic functions from equation (4.4) (without the $f_{i}^{\text {evo }}$ functions, which are provided by $\mathrm{RTE}^{1}$ ). Figure 4.1 illustrates these estimates for the demand process and the coal capacity process.

\section{Demand seasonalities}

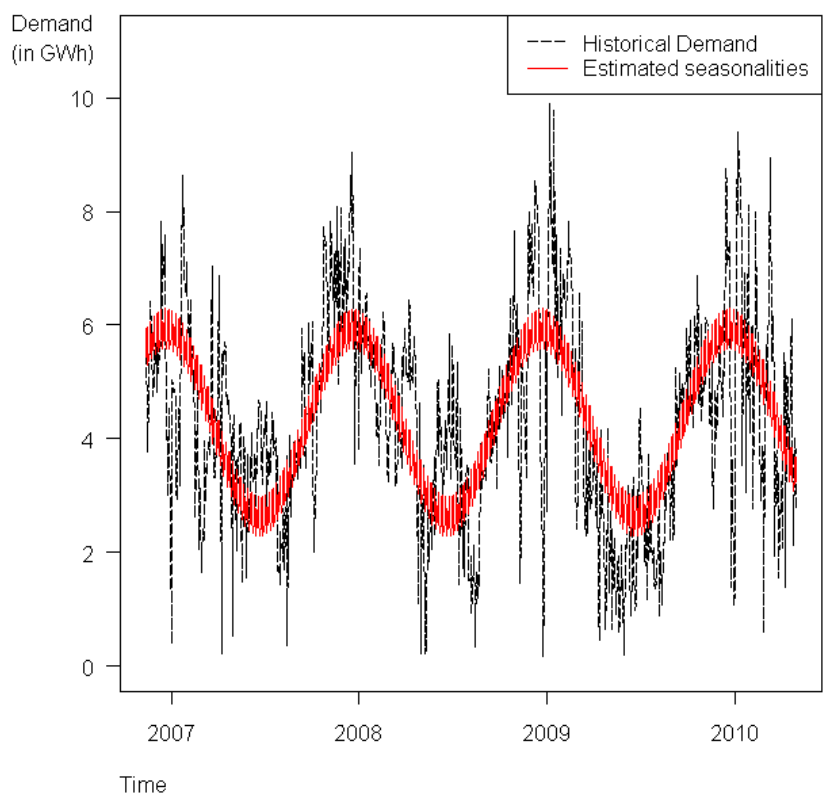

(a) Demand

\section{Coal capacity seasonality}

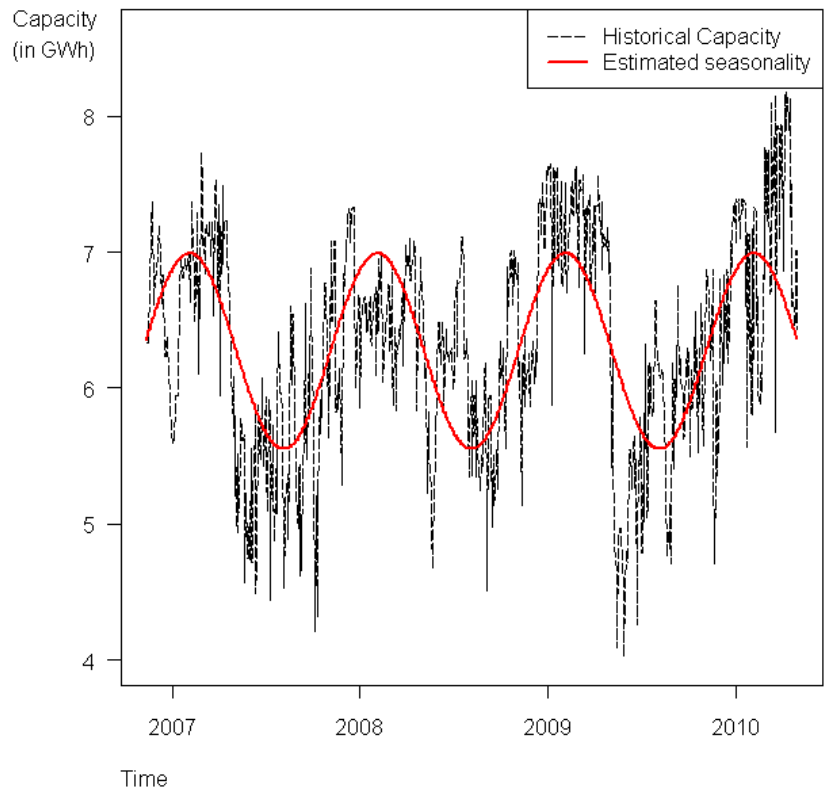

(b) Coal capacity

Figure 4.1: Seasonalities estimates

All parameters are statistically significant. After doing that, one can subtract the estimated seasonalities from the data and work with the resulting series, that correspond to realizations of the processes $Z_{D}(t)$ and $Z_{i}(t)$ as in equation (4.1). We estimate the mean-reversion parameters $\alpha_{D}$ and $\alpha_{i}, 1 \leq i \leq n$, by exploiting the link between continuous OU processes and discrete $\operatorname{AR}(1)$ processes.

\footnotetext{
${ }^{1}$ The French transmission system operator.
} 
Indeed, applying the Euler scheme to SDEs (4.2), denoting $\Delta t$ as the time stepsize, yields:

$$
\begin{aligned}
Z_{D}(t+\Delta t) & =Z_{D}(t)\left(1-\alpha_{D} \Delta t\right)+b \sqrt{\Delta t} N_{D}(t) \\
Z_{i}(t+\Delta t) & =Z_{i}(t)\left(1-\alpha_{i} \Delta t\right)+\beta_{i} \sqrt{\Delta t} N_{i}(t)
\end{aligned}
$$

for each time step $t$, where $N_{D}(t)$ and $N_{i}(t)$ are independent standard Gaussian white noises. Consequently, simple linear regressions will yield estimates for $\alpha_{D}, b, \alpha_{i}$ and $\beta_{i}, 1 \leq i \leq n$. From the different hypothesis of the model, a statistical analysis of the residuals reveals that the least accurate is the Gaussian hypothesis, as a slight but statistically significant excess kurtosis appears. This phenomenon is illustrated by Figure 4.2, where the residual densities of $Z_{D}$ and $Z_{1}$, estimated by the kernel method, are compared to Gaussian distributions with the same mean and the same variance. Nevertheless, given the moderate excess kurtosis estimates (around 2.0, see Table 1 ) and the tractability provided by the presence of Brownian motions, we choose to stick to the model built so far. Possible extensions of the model may later accomodate these empirical deviations.

Demand: Residual density vs. Gaussianity

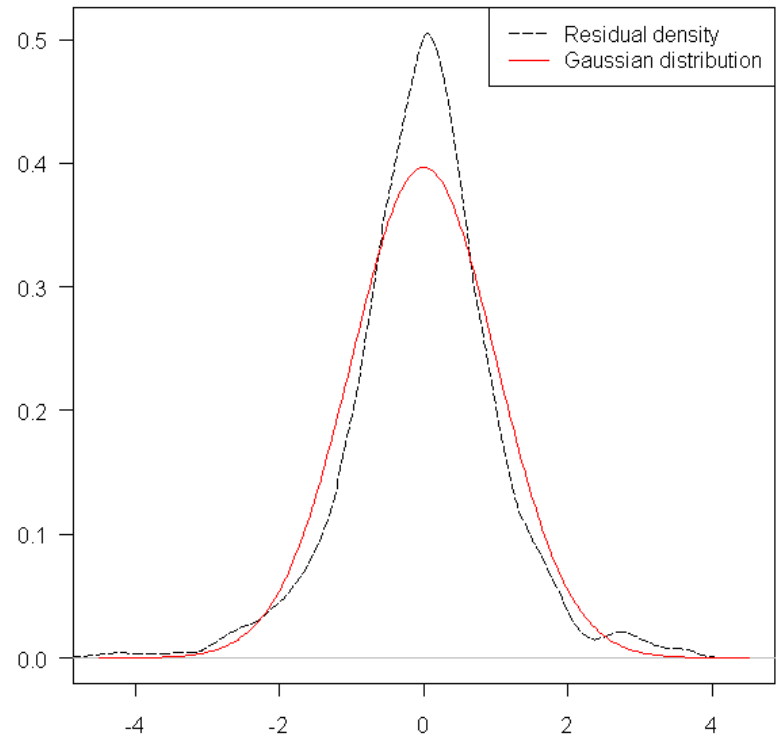

Zo (in GWh)

\section{Coal capacity: Residual density vs. Gaussianity}

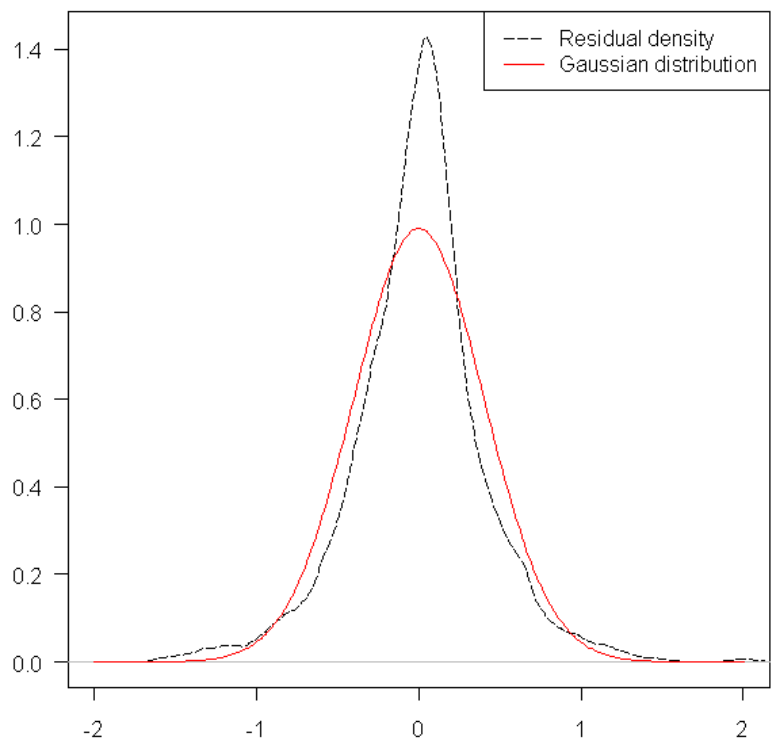

$Z_{1}$ (in GWh)

(a) Demand

(b) Coal capacity

Figure 4.2: Residuals estimated densities

\begin{tabular}{lllll}
\hline residuals & mean & st.dev. & skewness & (excess) kurtosis \\
\hline \hline demand & 0.00 & 1.01 & -0.13 & 1.92 \\
\hline coal & 0.00 & 0.40 & -0.04 & 2.08 \\
\hline
\end{tabular}

Table 1: Moments Estimates

\subsection{Computing the Conditional Expectation of Scarcity Function}

Using the dynamics of demand and capacities set in Section 4.1, we can now study in detail the conditional expectation of scarcity function $G_{i}^{T}\left(t, C_{t}, D_{t}\right)$ and its partial derivatives, which are key quantities for pricing and hedging electricity derivatives (see Section 3). We will see how they can be mathematically and numerically computed. 


\subsubsection{Definition of the auxiliary function $G(m, \sigma)$}

We recall from Section 3.3.1 the definition of the CES function:

$$
G_{i}^{T}\left(t, C_{t}, D_{t}\right)=\mathbb{E}\left[g\left(C_{T}^{\max }-D_{T}\right) \mathbf{1}_{\left\{D_{T} \in I_{T}^{i}\right\}} \mid \mathcal{F}_{t}^{D, C}\right], \quad 1 \leq i \leq n
$$

Recall that the change of measure from $\mathbb{P}$ to the minimal EMM $\widehat{\mathbb{Q}}$ does not impact the processes $D$ and $C^{i}, 1 \leq i \leq n$, meaning that the two measures $\widehat{\mathbb{Q}}$ and $\mathbb{P}$ coincide on $\mathcal{F}_{t}^{D, C}$ for any $t$. Therefore, these processes and their dynamics are still defined by equations (4.1) and (4.2), which are easily solved to deduce the laws of $D_{T} \mid \mathcal{F}_{t}^{D, C}$ and $C_{T}^{i} \mid \mathcal{F}_{t}^{D, C}$ :

Proposition 4.1. Conditionally on $\mathcal{F}_{t}^{D, C}$, the random variable $D_{T}$ is Gaussian, i.e. $D_{T} \mid \mathcal{F}_{t}^{D, C} \sim$ $\mathcal{N}\left(m_{t, T}^{D}, \sigma_{t, T}^{D}\right)$ where:

$$
\begin{aligned}
m_{t, T}^{D} & =f_{D}(T)+e^{-\alpha_{D}(T-t)}\left(D_{t}-f_{D}(t)\right) \\
\left(\sigma_{t, T}^{D}\right)^{2} & =\frac{b^{2}}{2 \alpha_{D}}\left[1-e^{-2 \alpha_{D}(T-t)}\right]
\end{aligned}
$$

Similarly, $C_{T}^{i} \mid \mathcal{F}_{t}^{D, C} \sim \mathcal{N}\left(m_{t, T}^{i}, \sigma_{t, T}^{i}\right)$ where:

$$
\begin{aligned}
m_{t, T}^{i} & =f_{i}(T)+e^{-\alpha_{i}(T-t)}\left(C_{t}^{i}-f_{i}(t)\right) \\
\left(\sigma_{t, T}^{i}\right)^{2} & =\frac{\beta_{i}^{2}}{2 \alpha_{i}}\left[1-e^{-2 \alpha_{i}(T-t)}\right]
\end{aligned}
$$

Proof. See Appendix B.1.

In view of equation (4.5), Proposition 4.1 indicates that the quantity of interest for computing the CES function is given by:

$$
\mathcal{G}(m, \sigma):=\mathbb{E}[g(X)]
$$

where $X \sim \mathcal{N}(m, \sigma)$ is a Gaussian random variable under the measure considered.

\subsubsection{Expressing the CES function via $\mathcal{G}(m, \sigma)$}

Indeed, one can express $G_{i}^{T}\left(t, C_{t}, D_{t}\right)$ using the auxiliary function $\mathcal{G}(m, \sigma)$. This is the purpose of this section. First, we consider the particular case with only one fuel $(n=1)$. The index $i$ can be dropped, and the result is the following:

Proposition 4.2. When $n=1$, we have :

$$
G^{T}\left(t, C_{t}, D_{t}\right)=\mathcal{G}(\bar{m}, \bar{\sigma}), \quad \bar{m}=m_{t, T}-m_{t, T}^{D}, \quad \bar{\sigma}^{2}=\left(\sigma_{t, T}\right)^{2}+\left(\sigma_{t, T}^{D}\right)^{2}
$$

Proof. See Appendix B.2.

In this particular case $n=1$, the link between the two functions is very simple. We now turn to the general case with $n$ fuels. The link is now given by the following proposition:

Proposition 4.3. For $2 \leq i \leq n-1$, we have

$$
\begin{aligned}
G_{i}^{T}\left(t, C_{t}, D_{t}\right) & =H\left(\bar{m}_{i+1}^{n}, \bar{m}_{1}^{i, D}, \bar{\sigma}_{i+1}^{n}, \bar{\sigma}_{1}^{i, D}\right)-H\left(\bar{m}_{i}^{n}, \bar{m}_{1}^{i-1, D}, \bar{\sigma}_{i}^{n}, \bar{\sigma}_{1}^{i-1, D}\right) \\
H\left(m_{1}, m_{2}, \sigma_{1}, \sigma_{2}\right) & :=\int_{0}^{\infty} \mathcal{G}\left(x+m_{1}, \sigma_{1}\right) \Phi_{N}\left(x ; m_{2}, \sigma_{2}\right) d x
\end{aligned}
$$

where $\Phi_{N}(x ; m, \sigma)$ is the probability density function of a normal random variable with mean $m$ and variance $\sigma^{2}>0$ and where:

$$
\begin{aligned}
\bar{m}_{i}^{n} & =\sum_{k=i}^{n} m_{t, T}^{k} & \left(\bar{\sigma}_{i}^{n}\right)^{2} & =\sum_{k=i}^{n}\left(\sigma_{t, T}^{k}\right)^{2} \\
\bar{m}_{1}^{i, D} & =\sum_{k=1}^{i} m_{t, T}^{k}-m_{t, T}^{D} & \left(\bar{\sigma}_{1}^{i, D}\right)^{2} & =\sum_{k=1}^{i}\left(\sigma_{t, T}^{k}\right)^{2}+\left(\sigma_{t, T}^{D}\right)^{2}
\end{aligned}
$$


For $i=1$ or $i=n, G_{i}^{T}\left(t, C_{t}, D_{t}\right)$ is given by:

$$
\begin{aligned}
& G_{1}^{T}\left(t, C_{t}, D_{t}\right)=H\left(\bar{m}_{2}^{n}, \bar{m}_{1}^{1, D}, \bar{\sigma}_{2}^{n}, \bar{\sigma}_{1}^{1, D}\right) \\
& G_{n}^{T}\left(t, C_{t}, D_{t}\right)=\mathcal{G}\left(\bar{m}_{1}^{n, D}, \bar{\sigma}_{1}^{n, D}\right)-H\left(\bar{m}_{n}^{n}, \bar{m}_{1}^{n-1, D}, \bar{\sigma}_{n}^{n}, \bar{\sigma}_{1}^{n-1, D}\right)
\end{aligned}
$$

Proof. See Appendix B.3.

Therefore, equations (4.9) and (4.12) indicate that computing the CES function reduces to compute the functions $\mathcal{G}(m, \sigma)$ and $H\left(m_{1}, m_{2}, \sigma_{1}, \sigma_{2}\right)$. This is why we devote the next sections to the evaluation of these quantities. We first consider $\mathcal{G}(m, \sigma)$, as computing $H\left(m_{1}, m_{2}, \sigma_{1}, \sigma_{2}\right)$ will involve results concerning $\mathcal{G}(m, \sigma)$.

\subsubsection{Computation of $\mathcal{G}(m, \sigma)$}

The following proposition corresponds to the first step in the calculation of $\mathcal{G}(m, \sigma)$.

Proposition 4.4. Let $\mathcal{G}(m, \sigma)=\mathbb{E}[g(X)]$ where $X \sim \mathcal{N}(m, \sigma)$ and $g(x)=\min \left\{M, \frac{\gamma}{x^{\nu}}\right\} \mathbf{1}_{\{x>0\}}+$ $M 1_{\{x \leq 0\}}$ with $M>0, \gamma>0, \nu>0$ and $\sigma>0$. Then $\mathcal{G}(m, \sigma)$ is given by the following expression:

$$
\mathcal{G}(m, \sigma)=M \mathcal{N}\left(\frac{\tilde{x}-m}{\sigma}\right)+\frac{\gamma}{(\sigma \sqrt{2})^{\nu} \sqrt{\pi}} \widetilde{\mathcal{G}}\left(\frac{\tilde{x}-m}{\sigma \sqrt{2}}, \frac{m}{\sigma \sqrt{2}} ; \nu\right)
$$

where $\tilde{x}=\left(\frac{\gamma}{M}\right)^{\frac{1}{\nu}}, \mathcal{N}$ is the cumulative distribution function of a standard normal random variable, and $\widetilde{\mathcal{G}}$ is defined by:

$$
\widetilde{\mathcal{G}}(x, y ; \nu):=\int_{x}^{\infty} \frac{1}{(y+z)^{\nu}} e^{-z^{2}} d z
$$

where $x+y \geq 0(x+y>0$ if $\nu \geq 1)$

Proof. See Appendix B.4.

The next step is to compute the function $\widetilde{\mathcal{G}}$ defined in equation (4.14).

\subsubsection{On the Extended Incomplete Goodwin-Staton integral}

We recall the definition of $\widetilde{\mathcal{G}}$ (equation (4.14)):

$$
\widetilde{\mathcal{G}}(x, y ; \nu)=\int_{x}^{\infty} \frac{1}{(y+z)^{\nu}} e^{-z^{2}} d z
$$

where $(x, y, \nu) \in \mathbb{R}^{3}$ with $x+y>0$ if $\nu>1$, and $x+y \geq 0$ otherwise. These constraints ensure that $\widetilde{G}$ is well-defined. In the particular case $\nu=1, \widetilde{\mathcal{G}}$ corresponds to the incomplete Goodwin-Staton integral (cf. Deaño and Temme [2010]). This is why we call $\widetilde{\mathcal{G}}$ the extended incomplete GoodwinStaton integral (henceforth EIGS integral). Note that this extension of the Goodwin-Staton integral is different from the usual Generalized Goodwin-Staton integral (as defined in Mamedov [2007] for instance). Proposition B.1 in Appendix B.5 provides a probabilistic interpretation of the EIGS integral using the density of the sum of two independent Pareto and Gaussian random variables.

Properties. We establish two useful properties verified by the EIGS integral.

Proposition 4.5. Whenever $\nu \neq 1$, the following recurrence relation holds:

$$
\widetilde{\mathcal{G}}(x, y ; \nu)=\frac{1}{1-\nu}\left(2 \widetilde{\mathcal{G}}(x, y ; \nu-2)-2 y \widetilde{\mathcal{G}}(x, y ; \nu-1)-(x+y)^{1-\nu} e^{-x^{2}}\right)
$$

Proof. See Appendix B.6. 
This recursive formula can be used, for instance, to compute $\widetilde{\mathcal{G}}(x, y ;-n), n \in \mathbb{N}$. Indeed, the initial values to start the recursion, $\widetilde{\mathcal{G}}(x, y ; 0)$ and $\widetilde{\mathcal{G}}(x, y ;-1)$ are given by:

$$
\widetilde{\mathcal{G}}(x, y ; 0)=\sqrt{\pi}[1-\mathcal{N}(x \sqrt{2})] \quad, \quad \widetilde{\mathcal{G}}(x, y ;-1)=y \widetilde{\mathcal{G}}(x, y ; 0)+\frac{1}{2} e^{-x^{2}}
$$

We will get advantage of this useful application later, in Section 4.2.6. Now, we establish the key property of the EIGS integral, which provides the ground for our evaluation algorithm.

Proposition 4.6. The following identity holds:

$$
\widetilde{\mathcal{G}}(x, y ; \nu)=\frac{1}{2} e^{-y^{2}} \Gamma\left(\frac{1-\nu}{2},(x+y)^{2} ;-2 y ;-\frac{1}{2}\right)
$$

where $\Gamma$ is the extended incomplete gamma function (cf. Chaudhry and Zubair [2002] p.266; henceforth EIG function):

$$
\Gamma(\alpha, x ; b ; \beta):=\int_{x}^{\infty} t^{\alpha-1} \exp \left(-t-b t^{-\beta}\right) d t
$$

where $x \geq 0$ and $(\alpha, b, \beta) \in \mathbb{R}^{3}$ are such that $\Gamma(\alpha, x ; b ; \beta)$ exists.

Proof. See Appendix B.7.

\subsubsection{Numerical computation of the EIGS integral}

As indicated by equation (4.13), $G(m, \sigma)$ is composed of two parts. The first part involves the cumulative distribution function $\mathcal{N}$, for which efficient evaluation algorithms already exist. The second part involves the EIGS integral (4.14), which is the quantity that remains to be computed. Therefore, we provide in this section an algorithm to compute the EIGS integral.

Series expansion of the EIG function. Our idea to compute the EIGS integral is based on equation (4.16): if one can compute the EIG function (4.17), then one can compute the EIGS integral (4.14). In fact, there exist efficient algorithms to compute the (upper) incomplete Gamma function (henceforth IG function) defined by:

$$
\Gamma(\alpha, x)=\int_{x}^{\infty} t^{\alpha-1} \exp (-t) d t
$$

where $x \geq 0(x>0$ if $\alpha \leq 0)$. However, this is not the case for the less standard EIG function. We therefore propose below a simple algorithm to compute efficiently this function. It is based on the following property (see Chaudhry and Zubair [2002] p.273):

Proposition 4.7. The EIG function has the following series expansion :

$$
\Gamma(\alpha, x ; b ; \beta)=\sum_{n=0}^{\infty} \Gamma(\alpha-n \beta, x) \frac{(-b)^{n}}{n !}
$$

Proof. Replace exp $\left(-b t^{-\beta}\right)$ in $(4.17)$ by its MacLaurin series expansion, and recall the definition of the IG function (equation (4.18)).

Consequently, a simple way to numerically evaluate $\Gamma(\alpha, x ; b ; \beta)$ is to compute the first terms of the sum (4.19) up to numerical convergence, using an already existing algorithm to compute $\Gamma(\alpha-n \beta, x)$. However, note that we only need to compute $\Gamma(\alpha, x ; b ; \beta)$ for $\beta=-\frac{1}{2}$ (see equation (4.16); other values for $\beta$ may require preliminary steps before exploiting equation (4.19), see Appendix B.8). In other words, in the sum (4.19), the terms $\Gamma\left(\alpha+\frac{n}{2}, x\right)$ are to be computed. To do so, the following recurrence relation of the IG function can be used:

$$
\Gamma(\alpha+1, x)=\alpha \Gamma(\alpha, x)+x^{\alpha} e^{-x}
$$

Thus, to compute the terms of the sum (4.19), the use of an IG evaluation algorithm will be necessary only for the two first IG terms $\Gamma(\alpha, x)$ and $\Gamma\left(\alpha+\frac{1}{2}, x\right)$, as the next IG terms can be computed using (4.20). Such an implementation is provided in Appendix C.1. 
Approximation for large positive $y$. Combining equations (4.16) and (4.19), we obtain the following identity:

$$
\widetilde{\mathcal{G}}(x, y ; \nu)=\frac{1}{2} e^{-y^{2}} \sum_{n=0}^{\infty} \Gamma\left(\frac{1-\nu}{2}+\frac{n}{2},(x+y)^{2}\right) \frac{(2 y)^{n}}{n !}
$$

As a consequence, the smaller $|y|$ is, the more efficient the computation of the EIGS integral is. Therefore, finding accurate approximations when $|y| \gg 0$ sounds useful. In fact, when $y>0$, such an approximation will not only be useful but also necessary, because in our case where $x+y \geq 0$ is constant (see equation (4.13)) while $y$ can take large positive values (see equation (4.9)), the decomposition (4.6) can involve the product of the very small term $\frac{1}{2} e^{-y^{2}}$ with the very large term $\Gamma\left(\frac{1-\nu}{2},(x+y)^{2} ;-2 y ;-\frac{1}{2}\right)$. For large $y$, it is possible that $\frac{1}{2} e^{-y^{2}}$ reaches numerically zero, leading to the wrong estimate $\widetilde{\mathcal{G}}(x, y ; \nu)=0$. This phenomenon is illustrated in Figure 4.3a, where the parameters have been chosen to be realistic in regard to the estimates in Section 4.1 and to the equations $(4.6),(4.7),(4.11)$ and the subsequent equations up to $\widetilde{G}$ and $\widetilde{H}$. For large positive $y$, we propose the following approximation:

$$
\widetilde{\mathcal{G}}(x, y ; \nu) \simeq \frac{1}{y^{\nu}} \exp \left(\frac{\nu^{2}}{4 y^{2}}\right) \sqrt{\pi}\left[1-\mathcal{N}\left(\sqrt{2} x+\frac{\nu}{\sqrt{2} y}\right)\right]
$$

Its derivation is detailed in Appendix B.9. Figure 4.3a illustrates this approximating function on an example, and compares it with the series expansion (4.21). The accuracy of (4.22) for large $y$ can be appreciated. Approximation (4.22) appears suitable to overcome the numerical problem described above.

$\tilde{\mathrm{G}}(1-\mathrm{y}, \mathrm{y} ; v=1)$

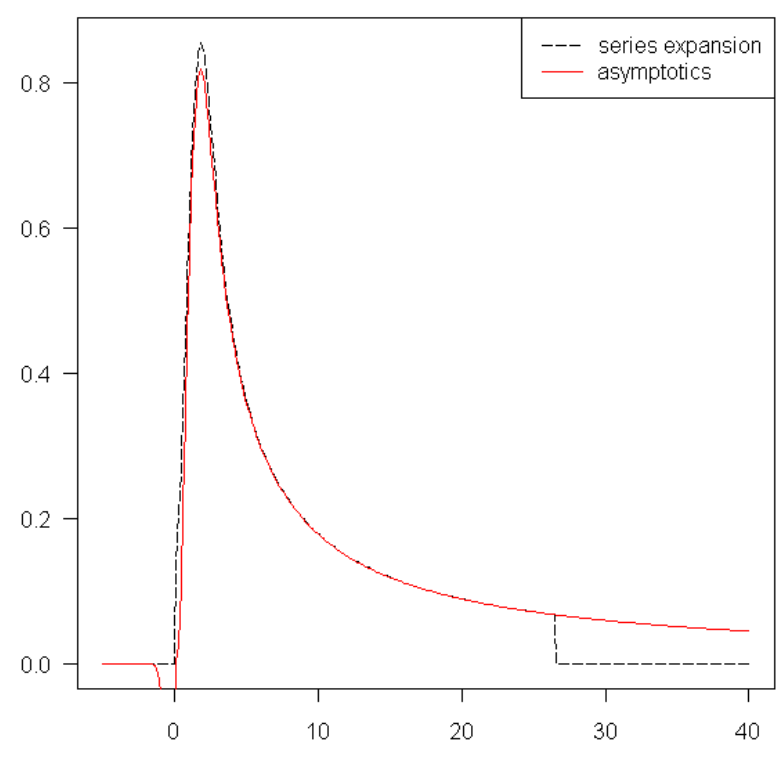

y

(a) Computing the EIGS integral
$\tilde{H}\left(m 1=2, m_{2}, \sigma_{1}=0.5, \sigma_{2}=1 ; v=1\right) \quad(\tilde{x}=0.1)$

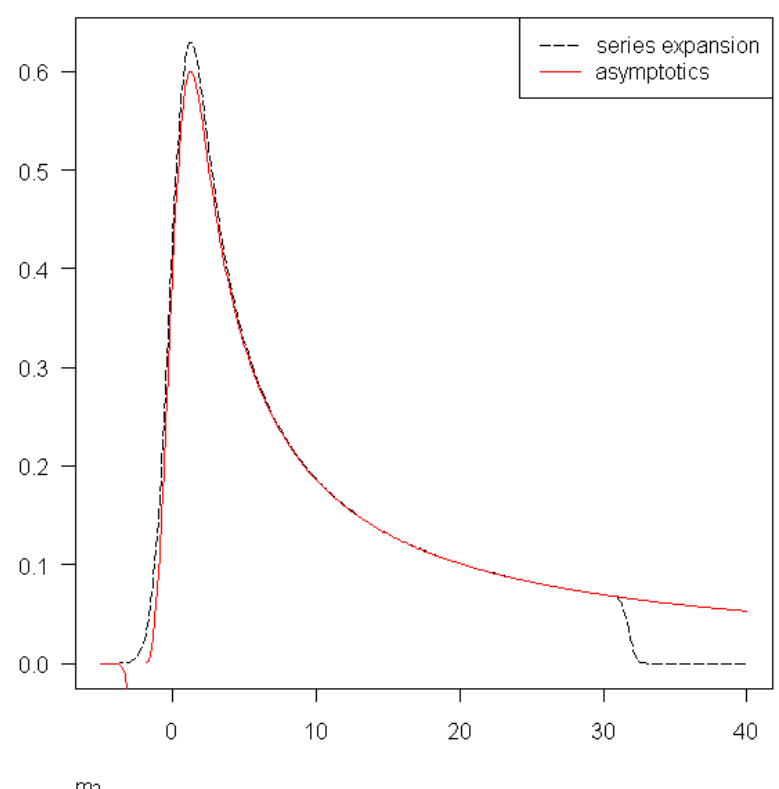

(b) Computing $\widetilde{H}$

Figure 4.3: Numerical approximations

Approximation for large negative $y$. When $y$ takes large negative values, the previous numerical difficulty does not appear, as both terms $\frac{1}{2} e^{-y^{2}}$ and $\Gamma\left(\frac{1-\nu}{2},(x+y)^{2} ;-2 y ;-\frac{1}{2}\right)$ become small. In fact, $\widetilde{\mathcal{G}}(x, y ; \nu)$ can readily reach zero numerically, and detecting when this occurs can save much computation time. A simple way to check it is to compute the upper bound provided below: 
Proposition 4.8. For any $y<0$ (and as usual $x+y \geq 0$ ):

$$
0 \leq \widetilde{\mathcal{G}}(x, y ; \nu) \leq e^{-y^{2}-(x+y)^{2}}(-2 y)^{\nu-1} \Gamma(1-\nu,(-2 y)(x+y))
$$

Proof. See Appendix B.10.

\subsubsection{Numerical computation of the CES function}

So far, we have been giving a method for computing $\mathcal{G}(m, \sigma)$. We have shown in Section 4.2.2, more precisely with equations (4.9) and (4.12), that the evaluation of the CES function $G_{i}^{T}\left(t, C_{t}, D_{t}\right)$ involves $\mathcal{G}(m, \sigma)$ through the quantity $H\left(m_{1}, m_{2}, \sigma_{1}, \sigma_{2}\right)$ defined in equation (4.10), recalled below:

$$
H\left(m_{1}, m_{2}, \sigma_{1}, \sigma_{2}\right)=\int_{0}^{\infty} \mathcal{G}\left(x+m_{1}, \sigma_{1}\right) \Phi_{N}\left(x ; m_{2}, \sigma_{2}\right) d x
$$

where $\left(m_{1}, m_{2}, \sigma_{1}, \sigma_{2}\right) \in \mathbb{R}^{4}$ with $\sigma_{1}>0, \sigma_{2}>0$. The following propositions are devoted to the computation of such a quantity.

Proposition 4.9. The following holds:

$$
\begin{aligned}
H\left(m_{1}, m_{2}, \sigma_{1}, \sigma_{2}\right)= & M \mathcal{N}\left(\frac{m_{2}}{\sigma_{2}}, \frac{\tilde{x}-m_{1}-m_{2}}{\sigma_{1}} ;\left[\begin{array}{ll}
0 & 0
\end{array}\right],\left[\begin{array}{cc}
1 & -\frac{\sigma_{2}}{\sigma_{1}} \\
-\frac{\sigma_{2}}{\sigma_{1}} & 1+\frac{\sigma_{2}^{2}}{\sigma_{1}^{2}}
\end{array}\right]\right) \\
& +\frac{\gamma}{\pi\left(\sigma_{1} \sqrt{2}\right)^{\nu}} \widetilde{H}\left(m_{1}, m_{2}, \sigma_{1}, \sigma_{2} ; \nu\right)
\end{aligned}
$$

where $\mathcal{N}\left(x, y ;\left[\begin{array}{ll}\mu_{1} & \mu_{2}\end{array}\right], \Sigma\right)$ is the cumulative distribution function of the bivariate Gaussian random vector with mean $\left[\begin{array}{ll}\mu_{1} & \mu_{2}\end{array}\right]$ and covariance matrix $\Sigma$, and $\widetilde{H}$ is defined by:

$$
\widetilde{H}\left(m_{1}, m_{2}, \sigma_{1}, \sigma_{2} ; \nu\right):=\int_{-\frac{m_{2}}{\sigma_{2} \sqrt{2}}}^{\infty} \widetilde{\mathcal{G}}\left(\frac{\tilde{x}-m_{1}-m_{2}-\sigma_{2} \sqrt{2} u}{\sigma_{1} \sqrt{2}}, \frac{m_{1}+m_{2}+\sigma_{2} \sqrt{2} u}{\sigma_{1} \sqrt{2}} ; \nu\right) e^{-u^{2}} d u
$$

Proof. See Appendix B.11.

The cumulative distribution function $\mathcal{N}\left(x, y ;\left[\begin{array}{ll}\mu_{1} & \mu_{2}\end{array}\right], \Sigma\right)$ is efficiently computable. The task is now to compute $\widetilde{H}\left(m_{1}, m_{2}, \sigma_{1}, \sigma_{2} ; \nu\right)$. This is done below.

\section{Series expansion.}

Proposition 4.10. $\widetilde{H}\left(m_{1}, m_{2}, \sigma_{1}, \sigma_{2} ; \nu\right)$ has the following series expansion:

$$
\widetilde{H}\left(m_{1}, m_{2}, \sigma_{1}, \sigma_{2} ; \nu\right)=\frac{\sigma_{1}}{2 \bar{\sigma}} e^{-\frac{\bar{m}^{2}}{2 \bar{\sigma}^{2}}} \sum_{n=0}^{\infty} \Gamma\left(\frac{1-\nu}{2}+\frac{n}{2}, \frac{\tilde{x}^{2}}{2 \sigma_{1}^{2}}\right) \widetilde{\mathcal{G}}\left(\frac{m_{1} \sigma_{2}^{2}-m_{2} \sigma_{1}^{2}}{\sigma_{1} \sigma_{2} \bar{\sigma} \sqrt{2}}, \frac{\bar{m} \sigma_{1}}{\sigma_{2} \bar{\sigma} \sqrt{2}},-n\right) \frac{\left(\frac{2 \sigma_{2}}{\bar{\sigma}}\right)^{n}}{n !}
$$

where $\bar{m}=m_{1}+m_{2}$ and $\bar{\sigma}=\sqrt{\sigma_{1}^{2}+\sigma_{2}^{2}}$.

Proof. See Appendix B.12.

The terms $\Gamma\left(\frac{1-\nu}{2}+\frac{n}{2}, \frac{\tilde{x}^{2}}{2 \sigma_{1}^{2}}\right)$ can be computed using the recurrence relation (4.20) (see the discussion following Proposition B.2), and the terms $\widetilde{\mathcal{G}}\left(\frac{m_{1} \sigma_{2}^{2}-m_{2} \sigma_{1}^{2}}{\sigma_{1} \sigma_{2} \bar{\sigma} \sqrt{2}}, \frac{\bar{m} \sigma_{1}}{\sigma_{2} \bar{\sigma} \sqrt{2}},-n\right)$ can be computed using the recurrence relation (4.15) (see the discussion following proposition 4.5). This provides an efficient way to compute $\widetilde{H}\left(m_{1}, m_{2}, \sigma_{1}, \sigma_{2} ; \nu\right)$, and hence the CES function $G_{i}^{T}\left(t, C_{t}, D_{t}\right)$ (via equations (4.9), (4.12) and (4.23)). Such an implementation is provided in Appendix C.3. 
Approximation for large positive $\bar{m}$. Equation (4.25) involves the coefficient exp $\left(-\frac{\bar{m}^{2}}{2 \bar{\sigma}^{2}}\right)$. Similarly to Section 4.2 .5 , this can prevent $\widetilde{H}$ from being correctly computed when $\bar{m}$ takes large positive values. In that case, the following approximation can be used:

$$
\widetilde{H}\left(m_{1}, m_{2}, \sigma_{1}, \sigma_{2} ; \nu\right) \simeq \frac{\pi\left(\sigma_{1} \sqrt{2}\right)^{\nu}}{\bar{m}^{\nu}} \exp \left(\frac{\nu^{2} \bar{\sigma}^{2}}{2 \bar{m}^{2}}\right) \mathcal{N}\left(\frac{m_{2}}{\sigma_{2}}, \frac{\bar{m}-\tilde{x}}{\sigma_{1}} ;\left[\begin{array}{cc}
\frac{\nu \sigma_{2}}{\bar{m}} & \frac{\nu \bar{\sigma}^{2}}{\sigma_{1} \bar{m}}
\end{array}\right],\left[\begin{array}{cc}
1 & \frac{\sigma_{2}}{\sigma_{1}} \\
\frac{\sigma_{2}}{\sigma_{1}} & 1+\frac{\sigma_{2}^{2}}{\sigma_{1}^{2}}
\end{array}\right]\right)
$$

The derivation of this expression is detailed in Appendix B.13. Figure 4.3b illustrates the approximation (4.26) on an example, where it is compared to the series expansion (4.25). Again, the approximation is quickly very accurate, and enables to consider large positive $\bar{m}$.

Approximation for large negative $\bar{m}$. Similarly to Section 4.2.5, we provide an upper bound useful for large negative $\bar{m}$.

Proposition 4.11. Suppose $\nu \geq 0$. Then the following holds:

$$
0 \leq \widetilde{H}\left(m_{1}, m_{2}, \sigma_{1}, \sigma_{2} ; \nu\right) \leq \pi\left(\frac{\sigma_{1} \sqrt{2}}{\tilde{x}}\right)^{\nu} \mathcal{N}\left(\frac{m_{2}}{\sigma_{2}} ;-\frac{\tilde{x}}{\sigma_{1}}+\frac{\bar{m}}{\sigma_{1}} ;\left[\begin{array}{ll}
0 & 0
\end{array}\right] ;\left[\begin{array}{cc}
1 & \frac{\sigma_{2}}{\sigma_{1}} \\
\frac{\sigma_{2}}{\sigma_{1}} & 1+\frac{\sigma_{2}^{2}}{\sigma_{1}^{2}}
\end{array}\right]\right)
$$

Proof. See Appendix B.14.

\subsubsection{Derivatives of the CES function}

Thanks to the various results established in the previous sections, we are now able to compute the partial derivatives of the CES function. They appear in the dynamics of the electricity forward prices (3.13) as well as in the hedging strategies of electricity derivatives (see Proposition 3.13).

Proposition 4.12. The following derivatives hold:

$$
\begin{aligned}
\frac{\partial \mathcal{G}}{\partial m}(m, \sigma) & =-\frac{\nu \gamma}{\sqrt{\pi}(\sigma \sqrt{2})^{\nu+1}} \widetilde{\mathcal{G}}\left(\frac{\tilde{x}-m}{\sigma \sqrt{2}}, \frac{m}{\sigma \sqrt{2}} ; \nu+1\right) \\
\frac{\partial H}{\partial m_{1}}\left(m_{1}, m_{2}, \sigma_{1}, \sigma_{2}\right) & =-\frac{\nu \gamma}{\pi\left(\sigma_{1} \sqrt{2}\right)^{\nu+1}} \widetilde{H}\left(m_{1}, m_{2}, \sigma_{1}, \sigma_{2} ; \nu+1\right) \\
\frac{\partial H}{\partial m_{2}}\left(m_{1}, m_{2}, \sigma_{1}, \sigma_{2}\right) & =\mathcal{G}\left(m_{1}, \sigma_{1}\right) \Phi_{N}\left(0 ; m_{2}, \sigma_{2}\right)-\frac{\nu \gamma}{\pi\left(\sigma_{1} \sqrt{2}\right)^{\nu+1}} \widetilde{H}\left(m_{1}, m_{2}, \sigma_{1}, \sigma_{2} ; \nu+1\right) \\
& =\mathcal{G}\left(m_{1}, \sigma_{1}\right) \Phi_{N}\left(0 ; m_{2}, \sigma_{2}\right)+\frac{\partial H}{\partial m_{1}}\left(m_{1}, m_{2}, \sigma_{1}, \sigma_{2}\right)
\end{aligned}
$$

These formulae allow to compute the derivatives of $G_{i}^{T}(t, x, y)$ :

For $2 \leq i \leq n-1$ and $1 \leq k \leq n$ :

$$
\begin{aligned}
\frac{\partial G_{i}^{T}}{\partial y}\left(t, C_{t}, D_{t}\right)= & -e^{-\alpha_{D}(T-t)}\left(\frac{\partial H}{\partial m_{2}}\left(\bar{m}_{i+1}^{n}, \bar{m}_{1}^{i, D}, \bar{\sigma}_{i+1}^{n}, \bar{\sigma}_{1}^{i, D}\right)-\frac{\partial H}{\partial m_{2}}\left(\bar{m}_{i}^{n}, \bar{m}_{1}^{i-1, D}, \bar{\sigma}_{i}^{n}, \bar{\sigma}_{1}^{i-1, D}\right)\right) \\
\frac{\partial G_{i}^{T}}{\partial x_{k}}\left(t, C_{t}, D_{t}\right)= & e^{-\alpha_{k}(T-t)}\left(\frac{\partial H}{\partial m_{1}}\left(\bar{m}_{i+1}^{n}, \bar{m}_{1}^{i, D}, \bar{\sigma}_{i+1}^{n}, \bar{\sigma}_{1}^{i, D}\right)+\mathcal{G}\left(\bar{m}_{i+1}^{n}, \bar{\sigma}_{i+1}^{n}\right) \Phi_{N}\left(0 ; \bar{m}_{1}^{i, D}, \bar{\sigma}_{1}^{i, D}\right) \mathbf{1}_{\{k \leq i\}}\right. \\
& \left.-\frac{\partial H}{\partial m_{1}}\left(\bar{m}_{i}^{n}, \bar{m}_{1}^{i-1, D}, \bar{\sigma}_{i}^{n}, \bar{\sigma}_{1}^{i-1, D}\right)-\mathcal{G}\left(\bar{m}_{i}^{n}, \bar{\sigma}_{i}^{n}\right) \Phi_{N}\left(0 ; \bar{m}_{1}^{i-1, D}, \bar{\sigma}_{1}^{i-1, D}\right) \mathbf{1}_{\{k<i\}}\right)
\end{aligned}
$$


For $i=1$ or $i=n$ :

$$
\begin{aligned}
\frac{\partial G_{1}^{T}}{\partial y}\left(t, C_{t}, D_{t}\right)= & -e^{-\alpha_{D}(T-t)} \frac{\partial H}{\partial m_{2}}\left(\bar{m}_{2}^{n}, \bar{m}_{1}^{1, D}, \bar{\sigma}_{2}^{n}, \bar{\sigma}_{1}^{1, D}\right) \\
\frac{\partial G_{1}^{T}}{\partial x_{k}}\left(t, C_{t}, D_{t}\right)= & e^{-\alpha_{k}(T-t)}\left(\frac{\partial H}{\partial m_{1}}\left(\bar{m}_{2}^{n}, \bar{m}_{1}^{1, D}, \bar{\sigma}_{2}^{n}, \bar{\sigma}_{1}^{1, D}\right)+\mathcal{G}\left(\bar{m}_{2}^{n}, \bar{\sigma}_{2}^{n}\right) \Phi_{N}\left(0 ; \bar{m}_{1}^{1, D}, \bar{\sigma}_{1}^{1, D}\right) \mathbf{1}_{\{k=1\}}\right) \\
\frac{\partial G_{n}^{T}}{\partial y}\left(t, C_{t}, D_{t}\right)= & -e^{-\alpha_{D}(T-t)}\left(\frac{\partial \mathcal{G}}{\partial m}\left(\bar{m}_{1}^{n, D}, \bar{\sigma}_{1}^{n, D}\right)-\frac{\partial H}{\partial m_{2}}\left(\bar{m}_{n}^{n}, \bar{m}_{1}^{n-1, D}, \bar{\sigma}_{n}^{n}, \bar{\sigma}_{1}^{n-1, D}\right)\right) \\
\frac{\partial G_{n}^{T}}{\partial x_{k}}\left(t, C_{t}, D_{t}\right)= & e^{-\alpha_{k}(T-t)}\left(\frac{\partial \mathcal{G}}{\partial m}\left(\bar{m}_{1}^{n, D}, \bar{\sigma}_{1}^{n, D}\right)-\frac{\partial H}{\partial m_{1}}\left(\bar{m}_{n}^{n}, \bar{m}_{1}^{n-1, D}, \bar{\sigma}_{n}^{n}, \bar{\sigma}_{1}^{n-1, D}\right)\right. \\
& \left.-\mathcal{G}\left(\bar{m}_{n}^{n}, \bar{\sigma}_{n}^{n}\right) \Phi_{N}\left(0 ; \bar{m}_{1}^{n-1, D}, \bar{\sigma}_{1}^{n-1, D}\right) \mathbf{1}_{\{k<n\}}\right)
\end{aligned}
$$

Proof. See Appendix B.15.

This ends our derivation of results and algorithms to compute $G_{i}^{T}\left(t, C_{t}, D_{t}\right)$ and its derivatives.

\subsection{Pricing and Hedging}

Finally, using the algorithms from Section 4.2 and our dataset, we briefly test and discuss the pricing and hedging of some of the derivatives analysed in Section 3.

\subsubsection{Electricity futures}

The simplest test to perform is the partial hedge of electricity futures using fuel futures. Recalling equations (3.11) and (3.13), one can see that the algorithms from Section 4.2 allow us to compute $F_{t}^{e}(T)$ and $d F_{t}^{e}(T)$, which are needed to simulate the partial hedge portfolio. We consider a 3-month electricity futures with a delivery period of one single hour. We use a constant rebalancing stepsize of one day, and neglect transaction costs. Figure 4.4a depicts the distribution of the final hedging error, estimated from a sample of 1000 realizations. For comparison, the distribution of the hedging error before maturity is represented as well, for different remaining maturities. 
Distribution of hedging error: Time evolution

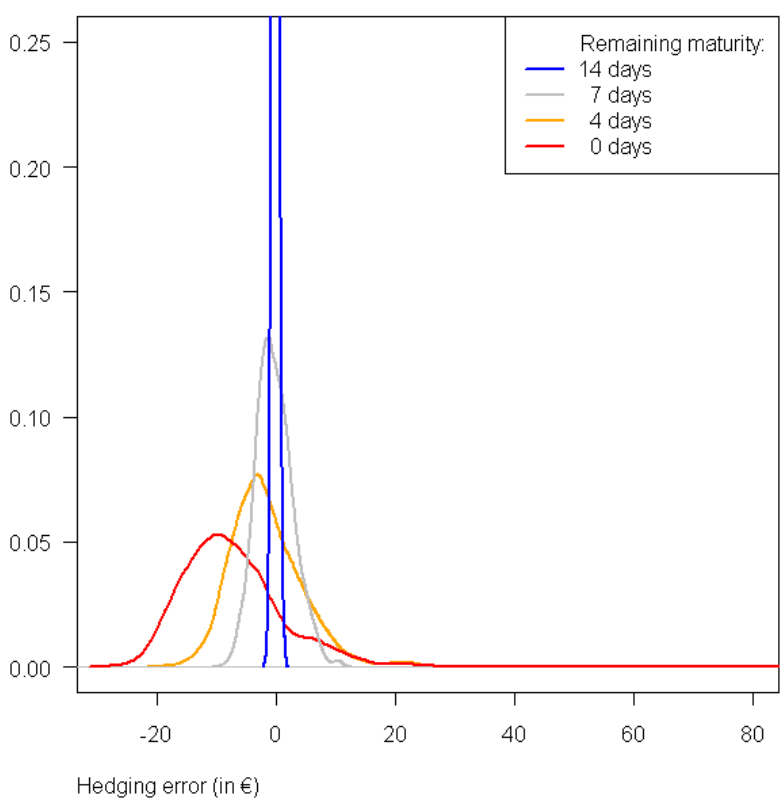

(a) Distribution

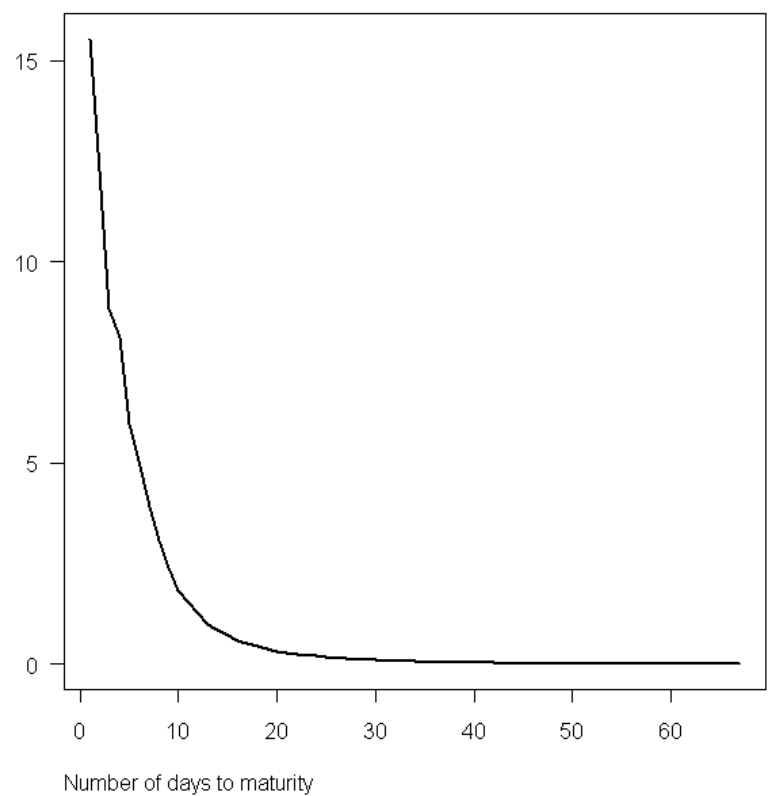

(b) Standard deviation

Figure 4.4: Hedging error

One can observe that up to two weeks before the expiration of the 3-month futures, the hedge generates hardly any error. However, during the last days of the product's life, the distribution of the error widens considerably. The standard deviation of the error, for instance, culminates at maturity, as shown by Figure 4.4b. Similarly, the asymetry of the error culminates at maturity, where a positive skewness of 8.7 is measured (The maximum hedging error at maturity reaches $212 €$ on this sample. For the sake of readability, the hedging error on Figure 4.4 a was limited to $80 €$ ). To sum up, depending on the time to maturity, two different behaviours can be observed:

- Far from maturity, the partial hedge is almost perfect. Indeed, recall equations (4.6) and (4.7). Because of the coefficients $e^{-\alpha_{D}(T-t)}$ and $e^{-\alpha_{i}(T-t)}$ (and the hypothesis of constant volatilities, see equation (4.3)), the weights $G_{i}^{T}\left(t, C_{t}, D_{t}\right)$ can be considered constant when $T-t \gg 0$. In this limit, the electricity futures are only driven by the fuel risks, ie. the electricity futures behaves like a basket of fuel futures. Consequently, the partial hedge turns out to be an almost perfect static hedge. With our estimates of $\alpha_{D}$ and $\alpha_{i}$ (around 70), this behaviour can be considered to hold up to two weeks before maturity.

- Close to maturity, the partial hedge is almost useless. Indeed, the coefficients $e^{-\alpha_{D}(T-t)}$ and $e^{-\alpha_{i}(T-t)}$ become no longer negligible, and consequently the unhedged risks stemming from demand and capacities start to drive the electricity futures. In fact, the partial derivatives of $G_{i}^{T}\left(t, C_{t}, D_{t}\right)$ can become huge, overwhelming the hedged fuel risks. It is such so that even if demand and capacities happened to be tradeable assets, the necessary use of discrete hedging would lead to hedging errors similar to Figure 4.4. This behaviour is analogous to classical barrier options close to expiry while close to the barrier, in which case static hedging is to be preferred to dynamic hedging.

\subsubsection{Spread options}

Finally, we look at the price of spread options obtained in our model. Using the results in Section 3.4.1, we compute the prices of options with payoff $\left(P_{T}-h_{1} S_{T}^{1}-K\right)^{+}, K>0$, i.e. European calls on dark spread with instantaneous delivery period. We compute equation (3.19) using numerical 
integration, replacing the integration on $\mathbb{R}^{3}$ (the spread $Y_{T}^{1}$ being the exponential of a Gaussian random variable) by an integration on the hypercube $[0,1]^{3}$ using the bijective transformations $y=$ $\frac{1}{1+\exp (-x)}$. Figure 4.5a illustrates the price surface for different strikes $K$ and different instantaneous maturities $T$.

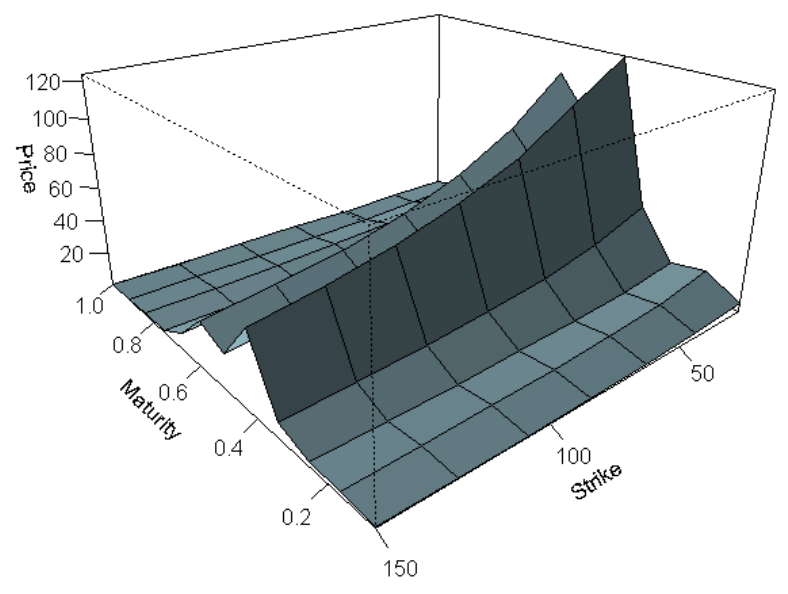

(a) Price surface

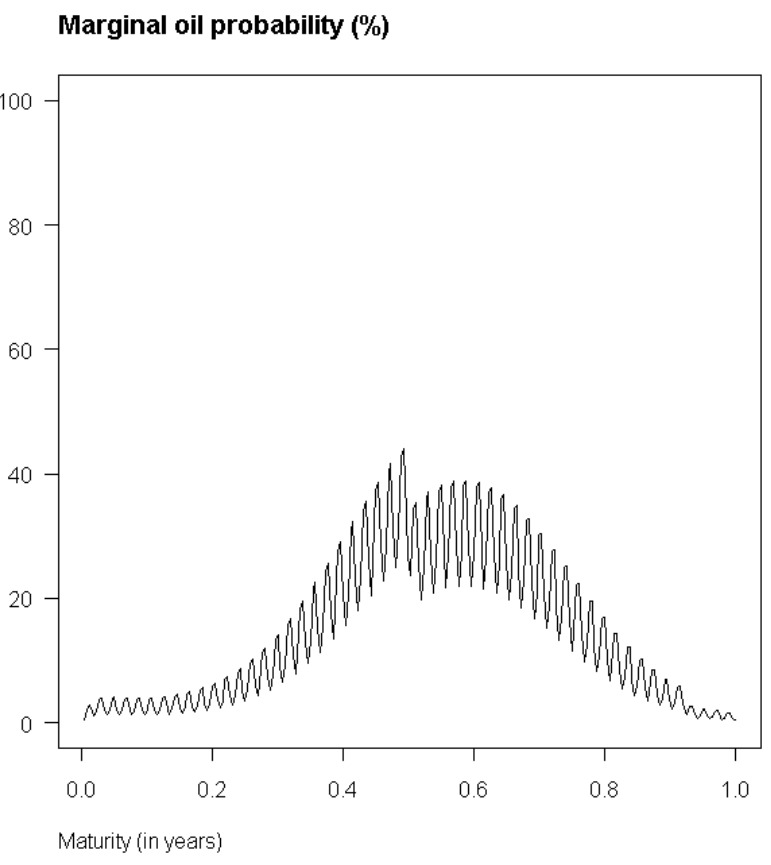

(b) Probability of marginal oil

Figure 4.5: Call on dark spread

The most visible effect is that of seasonality. The shape of the seasonality is driven by the quantity $\mathbb{P}\left(D_{T}>C_{T}^{1}\right)$, which represents the probability of using the most expensive fuel $S_{T}^{2}$ to produce electricity at time $T$. This quantity is depicted on Figure $4.5 \mathrm{~b}$ for comparison. Recall the weekly seasonality on demand and the deterministic evolution of installed capacities (equation (4.4)). Here the middle of the time period covers the next winter, where the likelihood of using the expensive oil is at its highest.

\section{Conclusion}

This paper is a contribution to the pricing of contingent claims on electricity markets. As opposed to the previous version of our model presented in Aid et al. [2009], the introduction of a scarcity function allows to capture the electricity spot price spikes at a cheap cost (only two parameters more, that can be easily estimated). The electricity spot price model developed here is particularly well suited for spread options on the spot since it is based on the economic relation that holds between fuel prices and electricity spot prices. Precise evaluation of electricity spread options are of main importance since they are the building blocks for the pricing of generation assets. Our structural risk-neutral model with scarcity function should enable us to assess in a near future the problem of the optimal timing of investment in generation assets.

Moreover, for the sake of simplicity, we considered in this work only diffusion processes. While this framework seems reasonable for such processes as demand, capacities, and some fuels like oil and coal, it may be too restrictive, for example, for the gas price, which is noticeably prone to spikes. However, enriching the dynamics of some fuels prices, for instance by adding a jump component, would not alter the general methodology developed in this paper. At the cost of more complex computations, 
it may refine the pricing of such electricity derivatives as spark spread options. This is a direction we plan to explore in our future research activities.

\section{References}

R. Aï, L. Campi, A. Nguyen Huu, and N. Touzi. A structural risk-neutral model of electricity prices. International Journal of Theoretical and Applied Finance, 12(7):925-947, 2009.

M. T. Barlow. A diffusion model for electricity prices. Mathematical Finance, 12(4):287-298, 2002.

G. Benmenzer, E. Gobet, and C. Jerusalem. Arbitrage free cointegrated models in gas and oil future markets. Technical report, GDF SUEZ and Laboratoire Jean Kuntzmann, 2007. available at http://hal.archives-ouvertes.fr/hal-00200422/fr/.

F. E. Benth and S. Koekebakker. Stochastic modeling of financial electricity contracts. Journal of Energy Economics, 30:1116-1157, 2007.

F. E. Benth and L. Vos. A multivariate non-gaussian stochastic volatility model with leverage for energy markets. Technical report, Department of Mathematics, University of Oslo, 2009.

F. E. Benth, L. Ekeland, R. Hauge, and B. F. Nielsen. A note on arbitrage-free pricing of forward contracts in energy markets. Applied Mathematical Finance, 10(4):325-336, 2003.

F. E. Benth, J. Kallsen, and T. Meyer-Brandis. A non-gaussian Ornstein-Uhlenbeck process for electricity spot price modeling and derivatives pricing. Applied Mathematical Finance, 14(2):153$169,2007$.

A. Boogert and D. Dupont. When supply meets demand: the case of hourly spot electricity prices. IEEE Transactions on Power Systems, 23(2):389-398, 2008. ISSN 0885-8950.

B. Bouchard and A. Nguyen Huu. No marginal arbitrage of the second kind for high production regimes in discrete time production-investment models with proportional transaction costs. Mathematical Finance, forthcoming, 2010.

M. Burger, B. Klar, A. Müller, and G. Schindlmayr. A spot market model for pricing derivatives in electricity markets. Quantitative Finance, 4:109-122, 2004.

A. Cartea and M. Figueroa. Pricing in electricity markets: a mean reverting jump diffusion model with seasonality. Applied Mathematical Finance, 12(4):313-335, 2005.

A. Cartea and P. Villaplana. Spot price modeling and the valuation of electricity forward contracts: the role of demand and capacity. Journal of Banking and Finance, 32:2501-2519, 2008.

M. A. Chaudhry and S. M. Zubair. On a class of incomplete gamma functions with applications. Chapman \& Hall, 2002.

L. Clewlow and C. Strickland. Energy Derivatives. Lacima Group, 2000.

M. Coulon and S. Howison. Stochastic behaviour of the electricity bid stack: from fundamental drivers to power prices. The Journal of Energy Markets, 2(1), 2009.

A. Deaño and N. M. Temme. Analytical and numerical aspects of a generalization of the complementary error function. Applied Mathematics and Computation, 216(12):3680-3693, 2010.

F. Delbaen, P. Monat, W. Schachermayer, M. Schweizer, and C. Stricker. Weighted norm inequalities and closedness of a space of stochastic integrals. Finance and Stochastics, 1:181-227, 1997.

S. Deng. Stochastic models of energy commodity prices and their applications: Mean-reversion with jumps and spikes. Technical Report PWP-073, University of California Energy Institute, 2000.

H. Föllmer and M. Schweizer. Hedging of contingent claims under incomplete information. Applied stochastic analysis (London, 1989), Stochastics Monogr., 5, Gordon and Breach, New York, pages 389-414, 1991. 
A. Friedman. Stochastic differential equations and applications. Academic Press New York, 1975.

N. Frikha and V. Lemaire. Joint modelling of gas and electricity spot prices. Technical report, LPMA, 2009.

H. Geman and V. Nguyen. Soybean inventory and forward curve dynamics. Management Science, pages 1076-1091, 2005. ISSN 0025-1909.

S. Goutte, N. Oudjane, and F. Russo. Variance optimal hedging for continuous time processes with independent increments and applications. Technical Report RR-FiME-09-09, Finance for Energy Market Research Centre, 2009.

D. Heath, E. Platen, and M. Schweizer. A comparison of two quadratic approaches to hedging in incomplete markets. Mathematical Finance, 11(4):385-413, 2001.

D. Hobson. Bounds for the utility-indifference prices of non-traded assets in incomplete markets. Decisons in Economics and Finance, 28:33-52, 2005.

T. Kanamura and K. Ohashi. A structural model for electricity prices with spikes: Measurement of spike risk and optimal policies for hydropower plant operation. Energy Economics, 29:1010-1032, 2007.

I. Karatzas and S. E. Shreve. Brownian motion and stochastic calculus. Springer-Verlag, 1991.

V. Kolodnyi. Valuation and hedging of european contingent claims on power with spikes: a nonmarkovian approach. Journal of Engineering Mathematics, 49(3):233-252, 2004.

D. Lautier and F. Raynaud. Statistical properties of derivatives: a journey in term structures. Physica A: Statistical Mechanics and its Applications, 2011. ISSN 0378-4371.

M. R. Lyle and R. J. Elliott. A "simple" hybrid model for power derivatives. Energy Economics, 31: 757-767, 2009.

B. A. Mamedov. Evaluation of the generalized Goodwin-Staton integral using binomial expansion theorem. Journal of Quantitative Spectroscopy \&3 Radiative Transfer, 105:8-11, 2007.

H. Pham. On quadratic hedging in continuous time. Math. Methods of Operations Research, 51: 315-229, 2000.

G. Pirrong and M. Jermakyan. The price of power - the valuation of power and weather derivatives. Technical report, Olin School of Business, 2000. available at SSRN: http://ssrn.com/abstract $=240815$.

G. Pirrong and M. Jermakyan. The price of power - the valuation of power and weather derivatives. Journal of Banking and Finance, 32(12):2520-2529, 2008.

D. Revuz and M. Yor. Continuous martingales and brownian motion. Springer Berlin Heidelberg, 1991.

E. Schwartz. The stochastic behavior of commodity prices: Implications for valuation and hedging. The Journal of Finance, 52(3):923-973, 1997.

M. Schweizer. A guided tour through quadratic hedging approaches. Option pricing, interest rates and risk management, Handb. Math. Finance, Cambridge Univ. Press, Cambridge, pages 538-574, 2001.

H. Working. Price relations between may and new-crop wheat futures at chicago since 1885. Food Research Institute Wheat Studies, 10:183-228, 1934. 


\section{A Dataset}

\section{A.1 Retrieving the residual demand}

RTE provides the following data, with their descriptions:

Demand:=Power Consumption+Network Losses -Pumping

Network Losses:=Power Production+Physical Import-Power Consumption-Physical Export

Interconnection:=Physical Export-Physical Import

(Incomplete) Production:=Hydro+Nuclear + Coal + Oil

In short, the following equations hold:

$$
\begin{aligned}
D & =C+L-\text { Pump } \\
L & =P-C-I \\
\widetilde{P} & =H+N+\text { Coal }+ \text { Oil }
\end{aligned}
$$

The two first equations yield:

$$
D+P u m p=P-I
$$

The available production data $\widetilde{P}$ covers, quoting RTE, "more than $90 \%$ of the generating units that are connected to the French transmission network". It covers therefore slightly less than the overall production $P$. Regarding the fact that RTE does not provide the production of the smallest units $(<20 \mathrm{MW})$, nor the production of non-hydroelectric renewables, we make the following reasonable assumption:

$$
P=R+H+N+C o a l+O i l=R+\widetilde{P}
$$

where $R$ denotes the production from non-hydroelectric renewable energies. Consequently, the residual demand can be retrieved as follows:

$$
\begin{aligned}
\text { Residual Demand } & :=(\text { Total Demand })-(R+H+N) \\
& =(D+I+\text { Pump })-(R+H+N) \\
& =P-R-H-N=\widetilde{P}-H-N
\end{aligned}
$$

Therefore computing the residual demand only requires $\widetilde{P}, H$ and $N$, which are available from the same RTE record file.

\section{A.2 Retrieving fuel capacity}

RTE provides records of the actual production and the effective availability for each fuel. What corresponds to our capacity variables $C_{t}^{i}, 1 \leq i \leq n$, is the effective availability. However, these data must be slightly adjusted first, as the comparison between actual production and effective availability reveals that even when electricity demand is at its highest, the actual production never reaches the effective availability. This phenomenon can be explained by the steady presence of primary and secondary reserves. For the accuracy of the model, in particular for the accurate detection of the marginal fuels, we correct this effect by the following adjustment:

$$
C_{t}^{1}:=0.94 \times(\text { coal effective availability }) \quad, \quad C_{t}^{2}:=0.80 \times(\text { oil effective availability })
$$

The two coefficients were inferred from the data. 


\section{B Proofs}

\section{B.1 Proposition 4.1}

Proof. SDEs (4.2) have classical explicit solutions, which combined with equations (4.1) yield at time $T$, starting from time $t$ :

$$
\begin{aligned}
& D_{T}=f_{D}(T)+e^{-\alpha_{D}(T-t)}\left(D_{t}-f_{D}(t)\right)+\int_{t}^{T} e^{-\alpha_{D}(T-s)} b d W_{t}^{D} \\
& C_{T}^{i}=f_{i}(T)+e^{-\alpha_{i}(T-t)}\left(C_{t}^{i}-f_{i}(t)\right)+\int_{t}^{T} e^{-\alpha_{i}(T-s)} \beta_{i} d W_{t}^{i}
\end{aligned}
$$

In particular, $D_{T}$ and $C_{T}^{i}$ are Gaussian random variables. Computing their expected values and their variances (using Itō's isometry) concludes the proof.

\section{B.2 Proposition 4.2}

Proof. When $n=1$, then $\left.I_{t}=\right]-\infty,+\infty[$, and consequently equation (4.5) becomes

$$
G^{T}\left(t, C_{t}, D_{t}\right)=\mathbb{E}\left[g\left(C_{T}-D_{T}\right) \mid \mathcal{F}_{t}^{D, C}\right] .
$$

We use Proposition 4.1 and the independence between the processes $C$ and $D$ to deduce that, conditionally to $\mathcal{F}_{t}^{D, C}, C_{T}-D_{T} \sim \mathcal{N}(\bar{m}, \bar{\sigma})$ where $\bar{m}=m_{t, T}-m_{t, T}^{D}$ and $\bar{\sigma}^{2}=\left(\sigma_{t, T}\right)^{2}+\left(\sigma_{t, T}^{D}\right)^{2}$. Therefore $G^{T}\left(t, C_{t}, D_{t}\right)=\mathbb{E}\left[g\left(C_{T}-D_{T}\right) \mid \mathcal{F}_{t}^{D, C}\right]=\mathcal{G}(\bar{m}, \bar{\sigma})$.

\section{B.3 Proposition 4.3}

Proof. For every $1 \leq i \leq j \leq n$, define $\bar{C}_{t}^{i, j}:=\sum_{k=i}^{j} C_{t}^{k}$. Thus, for $2 \leq i \leq n-1$ :

$$
\begin{aligned}
G_{i}^{T}\left(t, C_{t}, D_{t}\right)= & \mathbb{E}\left[g\left(\bar{C}_{T}^{1, n}-D_{T}\right) \mathbf{1}_{\left\{D_{T} \in I_{T}^{i}\right\}} \mid \mathcal{F}_{t}^{D, C}\right]=\mathbb{E}\left[g\left(\bar{C}_{T}^{1, n}-D_{T}\right) \mathbf{1}_{\left\{\bar{C}_{T}^{1, i-1} \leq D_{T}<\bar{C}_{T}^{1, i}\right\}} \mid \mathcal{F}_{t}^{D, C}\right] \\
= & \mathbb{E}\left[g\left(\bar{C}_{T}^{1, n}-D_{T}\right) \mathbf{1}_{\left\{D_{T}<\bar{C}_{T}^{1, i}\right\}} \mid \mathcal{F}_{t}^{D, C}\right]-\mathbb{E}\left[g\left(\bar{C}_{T}^{1, n}-D_{T}\right) \mathbf{1}_{\left\{D_{T} \leq \bar{C}_{T}^{1, i-1}\right\}} \mid \mathcal{F}_{t}^{D, C}\right] \\
= & \mathbb{E}\left[g\left(\left(\bar{C}_{T}^{1, i}-D_{T}\right)+\bar{C}_{T}^{i+1, n}\right) \mathbf{1}_{\left\{\bar{C}_{T}^{1, i}-D_{T}>0\right\}} \mid \mathcal{F}_{t}^{D, C}\right] \\
& -\mathbb{E}\left[g\left(\left(\bar{C}_{T}^{1, i-1}-D_{T}\right)+\bar{C}_{T}^{i, n}\right) \mathbf{1}_{\left\{\bar{C}_{T}^{1, i-1}-D_{T} \geq 0\right\}} \mid \mathcal{F}_{t}^{D, C}\right]
\end{aligned}
$$

Using the same arguments as in Section B.2, $\bar{C}_{T}^{1, i}-D_{T} \sim \mathcal{N}\left(\bar{m}_{1}^{i, D}, \bar{\sigma}_{1}^{i, D}\right)$ where $\bar{m}_{1}^{i, D}=\sum_{k=1}^{i} m_{t, T}^{k}-$ $m_{t, T}^{D}$ and $\left(\bar{\sigma}_{1}^{i, D}\right)^{2}=\sum_{k=1}^{i}\left(\sigma_{t, T}^{k}\right)^{2}+\left(\sigma_{t, T}^{D}\right)^{2}$. Similarly, $\bar{C}_{T}^{i+1, n} \sim \mathcal{N}\left(\bar{m}_{i+1}^{n}, \bar{\sigma}_{i+1}^{n}\right)$ where $\bar{m}_{i+1}^{n}=$ $\sum_{k=i+1}^{n} m_{t, T}^{k}$ and $\left(\bar{\sigma}_{i+1}^{n}\right)^{2}=\sum_{k=i+1}^{n}\left(\sigma_{t, T}^{k}\right)^{2}$. Consequently:

$$
\begin{aligned}
& \mathbb{E}\left[g\left(\left(\bar{C}_{T}^{1, i}-D_{T}\right)+\bar{C}_{T}^{i+1, n}\right) \mathbf{1}_{\left\{\bar{C}_{T}^{1, i}-D_{T} \geq 0\right\}} \mid \mathcal{F}_{t}^{D, C}\right] \\
= & \mathbb{E}\left[\mathbb{E}\left[g\left(\left(\bar{C}_{T}^{1, i}-D_{T}\right)+\bar{C}_{T}^{i+1, n}\right) \mathbf{1}_{\left\{\bar{C}_{T}^{1, i}-D_{T} \geq 0\right\}} \mid \bar{C}_{T}^{1, i}-D_{T}, \mathcal{F}_{t}^{D, C}\right] \mid \mathcal{F}_{t}^{D, C}\right] \\
= & \mathbb{E}\left[\mathcal{G}\left(\left(\bar{C}_{T}^{1, i}-D_{T}\right)+\bar{m}_{i+1}^{n}, \bar{\sigma}_{i+1}^{n}\right) \mathbf{1}_{\left\{\bar{C}_{T}^{1, i}-D_{T} \geq 0\right\}} \mid \mathcal{F}_{t}^{D, C}\right] \\
= & \int_{0}^{\infty} \mathcal{G}\left(x+\bar{m}_{i+1}^{n}, \bar{\sigma}_{i+1}^{n}\right) \Phi_{N}\left(x ; \bar{m}_{1}^{i, D}, \bar{\sigma}_{1}^{i, D}\right) d x
\end{aligned}
$$

where $\Phi_{N}(x ; m, \sigma)$ is the density of a Gaussian random variable with mean $m$ and variance $\sigma^{2}>0$. Performing the same calculation for the second expected value of equation (B.2), we get, for $2 \leq i \leq$ $n-1$, the desired result. In the cases when $i=1$ or $i=n$, few simplificating adjustments from the previous calculation give the desired results. 


\section{B.4 Proposition 4.4}

Proof. The random variable $X \sim \mathcal{N}(m, \sigma)$ can be written as $X=m+\sigma N$ where $N \sim \mathcal{N}(0,1)$ is a standard normal variable. Thus $\mathcal{G}(m, \sigma)=\mathbb{E}[g(X)]=\mathbb{E}[g(m+\sigma N)]$. Now, we remark that the function $g$ can be written in the following way:

$$
g(x)=\frac{\gamma}{x^{\nu}} \mathbf{1}_{\{x>\tilde{x}\}}+M \mathbf{1}_{\{x \leq \tilde{x}\}}
$$

where $\tilde{x}=\left(\frac{\gamma}{M}\right)^{\frac{1}{\nu}}$. Therefore:

$$
\begin{aligned}
\mathcal{G}(m, \sigma) & =\mathbb{E}[g(m+\sigma N)]=\int_{\mathbb{R}} g(m+\sigma x) \frac{1}{\sqrt{2 \pi}} e^{-\frac{x^{2}}{2}} d x \\
& =\int_{-\infty}^{\frac{\tilde{x}-m}{\sigma}} M \frac{1}{\sqrt{2 \pi}} e^{-\frac{x^{2}}{2}} d x+\int_{\frac{\tilde{x}-m}{\sigma}}^{\infty} \frac{\gamma}{(m+\sigma x)^{\nu}} \frac{1}{\sqrt{2 \pi}} e^{-\frac{x^{2}}{2}} d x \\
& =M \mathcal{N}\left(\frac{\tilde{x}-m}{\sigma}\right)+\frac{\gamma}{\sigma^{\nu} \sqrt{2 \pi}} \int_{\frac{\tilde{x}-m}{\sigma}}^{\infty} \frac{1}{\left(\frac{m}{\sigma}+x\right)^{\nu}} e^{-\frac{x^{2}}{2}} d x \\
& =M \mathcal{N}\left(\frac{\tilde{x}-m}{\sigma}\right)+\frac{\gamma}{(\sigma \sqrt{2})^{\nu} \sqrt{\pi}} \widetilde{\mathcal{G}}\left(\frac{\tilde{x}-m}{\sigma \sqrt{2}}, \frac{m}{\sigma \sqrt{2}} ; \nu\right)
\end{aligned}
$$

where $\widetilde{\mathcal{G}}$ is defined by equation (4.14).

\section{B.5 Interpretation of the EIGS integral}

The EIGS integral has some probabilistic interpretations. The following proposition provides one of them, involving Pareto distributions.

Proposition B.1. If $\nu>1$ and $x+y>0$, then:

$$
\widetilde{\mathcal{G}}(x, y ; \nu)=\frac{\sqrt{\pi}}{(\nu-1)(x+y)^{\nu-1}} \Phi_{N+P}(\sqrt{2} y)
$$

where $P \sim \operatorname{Par}(\nu-1, \sqrt{2}(x+y))$ is a Pareto random variable, $N \sim \mathcal{N}(0,1)$ is a standard Gaussian random variable independent of $P$, and $\Phi_{N+P}$ is the density of the sum $N+P$.

Proof. We recall that, for $\alpha>0$ and $x_{m}>0$, the probability density function of the Pareto random variable $\operatorname{Par}\left(\alpha, x_{m}\right)$ is given by $\Phi_{P}\left(x ; \alpha, x_{m}\right)=\frac{\alpha x_{m}^{\alpha}}{x^{\alpha+1}} \mathbf{1}_{\left\{x>x_{m}\right\}}$. When $\nu>1$ and $x+y>0$, this Pareto density can be introduced as follows:

$$
\begin{aligned}
\widetilde{\mathcal{G}}(x, y ; \nu) & =\int_{x}^{\infty} \frac{1}{(y+z)^{\nu}} e^{-z^{2}} d z=\int_{\sqrt{2}(x+y)}^{\infty} \frac{1}{\left(\frac{u}{\sqrt{2}}\right)^{\nu}} e^{-\left(\frac{u}{\sqrt{2}}-y\right)^{2}} \frac{d u}{\sqrt{2}} \\
& =\frac{1}{(\nu-1) \sqrt{2}(x+y)^{\nu-1}} \int_{\mathbb{R}} \Phi_{P}(u ; \nu-1, \sqrt{2}(x+y)) e^{-\frac{(\sqrt{2} y-u)^{2}}{2}} d u \\
& =\frac{\sqrt{\pi}}{(\nu-1)(x+y)^{\nu-1}} \int_{\mathbb{R}} \Phi_{P}(u ; \nu-1, \sqrt{2}(x+y)) \Phi_{N}(\sqrt{2} y-u ; 0,1) d u \\
& =\frac{\sqrt{\pi}}{(\nu-1)(x+y)^{\nu-1}} \Phi_{N+P}(\sqrt{2} y)
\end{aligned}
$$

where $\Phi_{N}(x ; m, \sigma)$ is the probability density function of a Gaussian random variable with mean $m$ and variance $\sigma^{2}$, and $\Phi_{N+P}$ is the probability density function of the sum of a standard Gaussian random variable $N \sim N(0,1)$ and a Pareto random variable $P \sim \operatorname{Par}(\nu-1, \sqrt{2}(x+y)), N$ and $P$ being independent. We have used the change of variable $u=(y+z) \sqrt{2}$ for the second equality. 


\section{B.6 Proposition 4.5}

Proof. Suppose $\nu \neq 1$. Then:

$$
\begin{aligned}
\widetilde{\mathcal{G}}(x, y ; \nu) & =\int_{x}^{\infty}\left((y+z)^{-\nu}\right)\left(e^{-z^{2}}\right) d z \\
& =\left[\frac{(y+z)^{1-\nu}}{1-\nu} e^{-z^{2}}\right]_{x}^{\infty}-\int_{x}^{\infty}\left(\frac{(y+z)^{1-\nu}}{1-\nu}\right)\left(-2 z e^{-z^{2}}\right) d z \\
& =-\frac{(x+y)^{1-\nu}}{1-\nu} e^{-x^{2}}+\frac{2}{1-\nu} \int_{x}^{\infty}(y+z)^{1-\nu} z e^{-z^{2}} d z \\
& =-\frac{(x+y)^{1-\nu}}{1-\nu} e^{-x^{2}}+\frac{2}{1-\nu} \int_{x+y}^{\infty} u^{1-\nu}(u-y) e^{-(u-y)^{2}} d u \\
& =-\frac{(x+y)^{1-\nu}}{1-\nu} e^{-x^{2}}+\frac{2}{1-\nu}\left[\int_{x+y}^{\infty} u^{2-\nu} e^{-(u-y)^{2}} d u-y \int_{x+y}^{\infty} u^{1-\nu} e^{-(u-y)^{2}} d u\right] \\
& =-\frac{(x+y)^{1-\nu}}{1-\nu} e^{-x^{2}}+\frac{2}{1-\nu}\left[\int_{x}^{\infty}(y+z)^{2-\nu} e^{-z^{2}} d z-y \int_{x}^{\infty}(y+z)^{1-\nu} e^{-z^{2}} d z\right] \\
& =-\frac{(x+y)^{1-\nu}}{1-\nu} e^{-x^{2}}+\frac{2}{1-\nu}[\widetilde{\mathcal{G}}(x, y ; \nu-2)-y \widetilde{\mathcal{G}}(x, y ; \nu-1)]
\end{aligned}
$$

\section{B.7 Proposition 4.6}

Proof. Using the definition of $\Gamma$ (equation (4.17)), we obtain:

$$
\begin{aligned}
& \Gamma\left(\frac{1-\nu}{2},(x+y)^{2} ;-2 y ;-\frac{1}{2}\right)=\int_{(x+y)^{2}}^{\infty} \frac{1}{t^{\frac{1+\nu}{2}}} \exp (-t+2 y \sqrt{t}) d t \\
= & e^{y^{2}} \int_{(x+y)^{2}}^{\infty} \frac{1}{t^{\frac{1+\nu}{2}}} \exp \left(-[\sqrt{t}-y]^{2}\right) d t=2 e^{y^{2}} \int_{|x+y|-y}^{\infty} \frac{1}{(y+z)^{\nu}} e^{-z^{2}} d t=2 e^{y^{2}} \widetilde{\mathcal{G}}(|x+y|-y, y ; \nu)
\end{aligned}
$$

where we performed the change of variable $z=\sqrt{t}-y$. If $x+y \geq 0$, then $|x+y|-y=x$ and we get (4.16). If $x+y<0$, then $|x+y|-y=-x-2 y$, and the change of variable $\{X=-x-2 y, Y=y\} \Leftrightarrow$ $\{x=-X-2 Y, y=Y\}$ gives:

$$
\begin{aligned}
\widetilde{\mathcal{G}}(X, Y ; \nu) & =\widetilde{\mathcal{G}}(-x-2 y, y ; \nu)=\frac{1}{2} e^{-y^{2}} \Gamma\left(\frac{1-\nu}{2},(x+y)^{2} ;-2 y ;-\frac{1}{2}\right) \\
& =\frac{1}{2} e^{-Y^{2}} \Gamma\left(\frac{1-\nu}{2},(-X-Y)^{2} ;-2 Y ;-\frac{1}{2}\right)=\frac{1}{2} e^{-Y^{2}} \Gamma\left(\frac{1-\nu}{2},(X+Y)^{2} ;-2 Y ;-\frac{1}{2}\right)
\end{aligned}
$$

and we have recovered (4.16). Interestingly, the relations holds whatever the sign of $x+y$ is. However, we recall that, to ensure the definition of $\tilde{G}(x, y ; \nu)$ for any $\nu$, we imposed $x+y \geq 0$ (see equation (4.14)). The proposition provides an extension to $x+y<0$, as long as the set of parameters ensure that the EIG function is well defined.

\section{B.8 Computing the EIG integral when $\beta<-1$}

It can be shown that the condition $\beta>-1$ is required for the convergence of the series expansion (4.19). Therefore, before exploiting this expansion, the following ordered preliminary steps are to be performed:

Proposition B.2. The following relations hold:

1. If $b=0$, then $\Gamma(\alpha, x ; 0 ; \beta)=\Gamma(\alpha, x)$ 
2. If $\beta=0$, then $\Gamma(\alpha, x ; b ; 0)=e^{-b} \Gamma(\alpha, x)$

3. If $\beta=-1$ then $\Gamma(\alpha, x ; b ;-1)= \begin{cases}\frac{1}{(b+1)^{\alpha}} \Gamma(\alpha,(b+1) x) & \text { if } b+1>0 \\ \frac{1}{(-\alpha) x^{-\alpha}} & \text { if } b+1=0, \alpha<0 \text { and } x>0 \\ +\infty & \text { else }\end{cases}$

4. If $\beta<-1$ and $b>0$, then $\Gamma(\alpha, x ; b ; \beta)=\frac{b^{\frac{\alpha}{\beta}}}{-\beta} \Gamma\left(-\frac{\alpha}{\beta}, b x^{-\beta} ; b^{\frac{1}{\beta}} ; \frac{1}{\beta}\right)$

Proof. The four relations are proved below:

1. $\Gamma(\alpha, x ; 0 ; \beta)=\int_{x}^{\infty} t^{\alpha-1} e^{-t} d t=\Gamma(\alpha, x)$

2. $\Gamma(\alpha, x ; b ; 0)=\int_{x}^{\infty} t^{\alpha-1} e^{-t-b} d t=e^{-b} \Gamma(\alpha, x)$

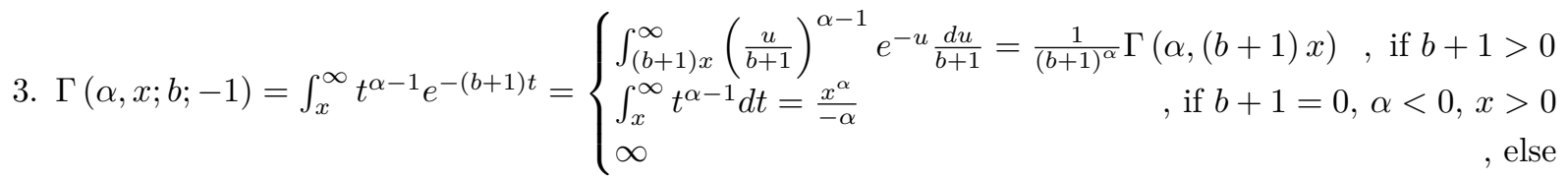

4. If $\beta<-1$ and $b>0$, the change of variable $u=b t^{-\beta}$ provides the result $\Gamma(\alpha, x ; b ; \beta)=$ $\frac{b^{\frac{\alpha}{\beta}}}{-\beta} \Gamma\left(-\frac{\alpha}{\beta}, b x^{-\beta} ; b^{\frac{1}{\beta}} ; \frac{1}{\beta}\right)$. More generally, the constraint $\beta<-1$ can be replaced by $\beta<0$, but when $\beta \in\left[-1,0\left[\right.\right.$, then $\frac{1}{\beta} \leq-1$ and thus the relation is of no help to compute $\Gamma(\alpha, x ; b ; \beta)$.

After these four preliminary checks, $\Gamma(\alpha, x ; b ; \beta)$ is either already computed, or is to be computed with $\beta>-1$ (as if $\beta<-1$ then $\frac{1}{\beta}>-1$ ), in which case the series expansion (4.19) can be exploited.

\section{B.9 Large $y$ asymptotics}

Let $y$ take large positive values:

$$
\begin{aligned}
\widetilde{\mathcal{G}}(x, y ; \nu) & =\int_{x}^{\infty} \frac{1}{(y+z)^{\nu}} e^{-z^{2}} d z=\frac{1}{y^{\nu}} \int_{x}^{\infty} \frac{1}{\left(1+\frac{z}{y}\right)^{\nu}} e^{-z^{2}} d z=\frac{1}{y^{\nu}} \int_{x}^{\infty} \exp \left(-\nu \ln \left(1+\frac{z}{y}\right)-z^{2}\right) d z \\
& \simeq \frac{1}{y^{\nu}} \int_{x}^{\infty} \exp \left(-\nu \frac{z}{y}-z^{2}\right) d z=\frac{1}{y^{\nu}} \exp \left(\frac{\nu^{2}}{4 y^{2}}\right) \int_{x}^{\infty} \exp \left(-\left(z+\frac{\nu}{2 y}\right)^{2}\right) d z \\
& =\frac{1}{y^{\nu}} \exp \left(\frac{\nu^{2}}{4 y^{2}}\right) \int_{x+\frac{\nu}{2 y}}^{\infty} e^{-z^{2}} d z=\frac{1}{y^{\nu}} \exp \left(\frac{\nu^{2}}{4 y^{2}}\right) \sqrt{\pi}\left[1-\mathcal{N}\left(\sqrt{2}\left(x+\frac{\nu}{2 y}\right)\right)\right]
\end{aligned}
$$

We have approximated $\ln \left(1+\frac{z}{y}\right)$ by its tangent in zero $\frac{z}{y}$ because of the Gaussian kernel that peaks at $z=0$, which is where precision is needed. Ultimately, we have approximated $\frac{1}{\left(1+\frac{z}{y}\right)^{\nu}}$ by $e^{-\nu \frac{z}{y}}$. It seems preferable to the tangent in zero of $\frac{1}{\left(1+\frac{z}{y}\right)^{\nu}}$ directly (namely $1-\nu \frac{z}{y}$ ) as $e^{-\nu \frac{z}{y}}$ behaves more like the initial function $\frac{1}{\left(1+\frac{z}{y}\right)^{\nu}}$. However, the difference, of course, vanishes for large $y$. 


\section{B.10 Proposition 4.8}

Proof. Let $y<0$ be given. We recall that, by definition of the function $\widetilde{\mathcal{G}}(x, y ; \nu)$ (see equation (4.14)), $x+y \geq 0$. Thus, using equations (4.16) and (4.17):

$$
\begin{aligned}
\widetilde{\mathcal{G}}(x, y ; \nu) & =\frac{1}{2} e^{-y^{2}} \Gamma\left(\frac{1-\nu}{2},(x+y)^{2} ;-2 y ;-\frac{1}{2}\right)=\frac{1}{2} e^{-y^{2}} \int_{(x+y)^{2}}^{\infty} t^{-\frac{1+\nu}{2}} \exp (-t+2 y \sqrt{t}) d t \\
& \leq \frac{1}{2} e^{-y^{2}} \exp \left(-(x+y)^{2}\right) \int_{(x+y)^{2}}^{\infty} t^{-\frac{1+\nu}{2}} \exp (2 y \sqrt{t}) d t \\
& =e^{-y^{2}-(x+y)^{2}}(-2 y)^{\nu-1} \int_{(-2 y)(x+y)}^{\infty} u^{-\nu} e^{-u} d u=e^{-y^{2}-(x+y)^{2}}(-2 y)^{\nu-1} \Gamma(1-\nu,(-2 y)(x+y))
\end{aligned}
$$

where we have performed the change of variable $u=-2 y \sqrt{t}$ for the next-to-last equality.

\section{B.11 Proposition 4.9}

Proof. Equation (4.13) yields:

$$
\begin{aligned}
& H\left(m_{1}, m_{2}, \sigma_{1}, \sigma_{2}\right)=\int_{0}^{\infty} \mathcal{G}\left(x+m_{1}, \sigma_{1}\right) \Phi_{N}\left(x ; m_{2}, \sigma_{2}\right) d x \\
& =\int_{0}^{\infty} \mathcal{G}\left(x+m_{1}, \sigma_{1}\right) \frac{1}{\sigma_{2} \sqrt{2 \pi}} \exp \left(-\frac{\left(x-m_{2}\right)^{2}}{2 \sigma_{2}^{2}}\right) d x \\
& =\int_{-\frac{m_{2}}{\sigma_{2} \sqrt{2}}}^{\infty} \mathcal{G}\left(m_{1}+m_{2}+\sigma_{2} \sqrt{2} u, \sigma_{1}\right) \frac{1}{\sqrt{\pi}} \exp \left(-u^{2}\right) d u \\
& =M \int_{-\frac{m_{2}}{\sigma_{2} \sqrt{2}}}^{\infty} \mathcal{N}\left(\frac{\tilde{x}-m_{1}-m_{2}-\sigma_{2} \sqrt{2} u}{\sigma_{1}}\right) \frac{1}{\sqrt{\pi}} e^{-u^{2}} d u \\
& +\frac{\gamma}{\pi\left(\sigma_{1} \sqrt{2}\right)^{\nu}} \int_{-\frac{m_{2}}{\sigma_{2} \sqrt{2}}}^{\infty} \widetilde{\mathcal{G}}\left(\frac{\tilde{x}-m_{1}-m_{2}-\sigma_{2} \sqrt{2} u}{\sigma_{1} \sqrt{2}}, \frac{m_{1}+m_{2}+\sigma_{2} \sqrt{2} u}{\sigma_{1} \sqrt{2}} ; \nu\right) e^{-u^{2}} d u \\
& =M \int_{-\infty}^{\frac{m_{2}}{\sigma_{2}}} \mathcal{N}\left(\frac{\tilde{x}-m_{1}-m_{2}+\sigma_{2} u}{\sigma_{1}}\right) \frac{1}{\sqrt{2 \pi}} e^{-\frac{u^{2}}{2}} d u+\frac{\gamma}{\pi\left(\sigma_{1} \sqrt{2}\right)^{\nu}} \widetilde{H}\left(m_{1}, m_{2}, \sigma_{1}, \sigma_{2} ; \nu\right)
\end{aligned}
$$

The integral above can be computed as follows:

$$
\begin{aligned}
\int_{-\infty}^{\frac{m_{2}}{\sigma_{2}}} \mathcal{N}\left(\frac{\tilde{x}-m_{1}-m_{2}+\sigma_{2} u}{\sigma_{1}}\right) \frac{1}{\sqrt{2 \pi}} e^{-\frac{u^{2}}{2}} d u & =\int_{-\infty}^{\frac{m_{2}}{\sigma_{2}}} \int_{-\infty}^{\frac{\tilde{x}-m_{1}-m_{2}+\sigma_{2} u}{\sigma_{1}}} \frac{1}{\sqrt{2 \pi}} e^{-\frac{z^{2}}{2}} d z \frac{1}{\sqrt{2 \pi}} e^{-\frac{u^{2}}{2}} d u \\
& =\frac{1}{2 \pi} \int_{-\infty}^{\frac{m_{2}}{\sigma_{2}}} \int_{-\infty}^{\frac{\tilde{x}-m_{1}-m_{2}}{\sigma_{1}}} \exp \left(-\frac{1}{2}\left[\left(v+\frac{\sigma_{2}}{\sigma_{1}} u\right)^{2}+u^{2}\right]\right) d v d u \\
& =\frac{1}{2 \pi} \int_{-\infty}^{\frac{m_{2}}{\sigma_{2}}} \int_{-\infty}^{\frac{\tilde{x}-m_{1}-m_{2}}{\sigma_{1}}} \exp \left(-\frac{1}{2}\left[\left(1+\frac{\sigma_{2}^{2}}{\sigma_{1}^{2}}\right) u^{2}+2 \frac{\sigma_{2}}{\sigma_{1}} u v+v^{2}\right]\right) d v d u \\
& =\frac{1}{2 \pi} \int_{-\infty}^{\frac{m_{2}}{\sigma_{2}}} \int_{-\infty}^{\frac{\tilde{x}-m_{1}-m_{2}}{\sigma_{1}}} \exp \left(-\frac{1}{2}\left[\begin{array}{ll}
u & v
\end{array}\right] \Sigma^{-1}\left[\begin{array}{l}
u \\
v
\end{array}\right]\right) d v d u
\end{aligned}
$$

where $\Sigma^{-1}=\left[\begin{array}{cc}1+\frac{\sigma_{2}^{2}}{\sigma_{1}^{2}} & \frac{\sigma_{2}}{\sigma_{1}} \\ \frac{\sigma_{2}}{\sigma_{1}} & 1\end{array}\right]$, and consequently $\Sigma=\left[\begin{array}{cc}1 & -\frac{\sigma_{2}}{\sigma_{2}} \\ -\frac{\sigma_{2}}{\sigma_{1}} & 1+\frac{\sigma_{2}^{2}}{\sigma_{1}^{2}}\end{array}\right]$. Noting that $|\Sigma|=1$, we have shown that $\int_{-\infty}^{\frac{m_{2}}{\sigma_{2}}} \mathcal{N}\left(\frac{\tilde{x}-m_{1}-m_{2}+\sigma_{2} u}{\sigma_{1}}\right) \frac{1}{\sqrt{2 \pi}} e^{-\frac{u^{2}}{2}} d u=\mathcal{N}\left(\frac{m_{2}}{\sigma_{2}}, \frac{\tilde{x}-m_{1}-m_{2}}{\sigma_{1}} ; 0,0, \Sigma\right)$, which concludes the proof. 


\section{B.12 Proposition 4.10}

Proof. Using equations (4.24) and (4.21), and using the notation $\bar{m}=m_{1}+m_{2}$ and $\bar{\sigma}=\sqrt{\sigma_{1}^{2}+\sigma_{2}^{2}}$ :

$$
\begin{aligned}
& \widetilde{H}\left(m_{1}, m_{2}, \sigma_{1}, \sigma_{2} ; \nu\right)=\int_{-\frac{m_{2}}{\sigma_{2} \sqrt{2}}}^{\infty} \widetilde{\mathcal{G}}\left(\frac{\tilde{x}-\bar{m}-\sigma_{2} \sqrt{2} u}{\sigma_{1} \sqrt{2}}, \frac{\bar{m}+\sigma_{2} \sqrt{2} u}{\sigma_{1} \sqrt{2}} ; \nu\right) e^{-u^{2}} d u \\
= & \int_{-\frac{m_{2}}{\sigma_{2} \sqrt{2}}}^{\infty} \frac{1}{2} e^{-\left(\frac{\bar{m}+\sigma_{2} \sqrt{2} u}{\sigma_{1} \sqrt{2}}\right)^{2}} \sum_{n=0}^{\infty} \Gamma\left(\frac{1-\nu}{2}+\frac{n}{2}, \frac{\tilde{x}^{2}}{2 \sigma_{1}^{2}}\right) \frac{\left(2 \frac{\bar{m}+\sigma_{2} \sqrt{2} u}{\sigma_{1} \sqrt{2}}\right)^{n}}{n !} e^{-u^{2}} d u \\
= & \sum_{n=0}^{\infty} \frac{1}{2} \Gamma\left(\frac{1-\nu}{2}+\frac{n}{2}, \frac{\tilde{x}^{2}}{2 \sigma_{1}^{2}}\right) \frac{2^{n}}{n !} \int_{-\frac{m_{2}}{\sigma_{2} \sqrt{2}}}^{\infty}\left(\frac{\bar{m}+\sigma_{2} \sqrt{2} u}{\sigma_{1} \sqrt{2}}\right)^{n} e^{-\left(\frac{\bar{m}+\sigma_{2} \sqrt{2} u}{\sigma_{1} \sqrt{2}}\right)^{2}-u^{2}} d u
\end{aligned}
$$

The integral can be computed as follows:

$$
\begin{aligned}
& \int_{-\frac{m_{2}}{\sigma_{2} \sqrt{2}}}^{\infty}\left(\frac{\bar{m}+\sigma_{2} \sqrt{2} u}{\sigma_{1} \sqrt{2}}\right)^{n} \exp \left(-\left(\frac{\bar{m}+\sigma_{2} \sqrt{2} u}{\sigma_{1} \sqrt{2}}\right)^{2}-u^{2}\right) d u \\
= & \int_{-\frac{m_{2}}{\sigma_{2} \sqrt{2}}}^{\infty}\left(\frac{\bar{m}+\sigma_{2} \sqrt{2} u}{\sigma_{1} \sqrt{2}}\right)^{n} \exp \left(-\frac{\bar{\sigma}^{2}}{\sigma_{1}^{2}}\left(u+\frac{\bar{m} \sigma_{2}}{\bar{\sigma}^{2} \sqrt{2}}\right)^{2}-\frac{\bar{m}^{2}}{2 \bar{\sigma}^{2}}\right) d u \\
= & e^{-\frac{\bar{m}^{2}}{2 \bar{\sigma}^{2}}} \frac{\sigma_{1}}{\bar{\sigma}} \int_{\frac{m_{1} \sigma_{2}^{2}-m_{2} \sigma_{1}^{2}}{\sigma_{1} \sigma_{2} \bar{\sigma} \sqrt{2}}}^{\infty}\left(\frac{\bar{m} \sigma_{1}}{\bar{\sigma}^{2} \sqrt{2}}+\frac{\sigma_{2}}{\bar{\sigma}} z\right)^{n} e^{-z^{2}} d z=e^{-\frac{\bar{m}^{2}}{2 \bar{\sigma}^{2}}} \frac{\sigma_{1}}{\bar{\sigma}}\left(\frac{\sigma_{2}}{\bar{\sigma}}\right)^{n} \widetilde{\mathcal{G}}\left(\frac{m_{1} \sigma_{2}^{2}-m_{2} \sigma_{1}^{2}}{\sigma_{1} \sigma_{2} \bar{\sigma} \sqrt{2}}, \frac{\bar{m} \sigma_{1}}{\sigma_{2} \bar{\sigma} \sqrt{2}},-n\right)
\end{aligned}
$$

where we have used the change of variable $z=\frac{\bar{\sigma}}{\sigma_{1}}\left(u+\frac{\bar{m} \sigma_{2}}{\bar{\sigma} 2 \sqrt{2}}\right)$ for the next-to-last equality. Inserting this formula into equation (B.4) yields the desired result.

\section{B.13 Large $\bar{m}$ asymptotics}

Let $\bar{m}$ take large positive values:

$$
\begin{aligned}
& \widetilde{H}\left(m_{1}, m_{2}, \sigma_{1}, \sigma_{2} ; \nu\right)=\int_{-\frac{m_{2}}{\sigma_{2} \sqrt{2}}}^{\infty} \widetilde{\mathcal{G}}\left(\frac{\tilde{x}-\bar{m}-\sigma_{2} \sqrt{2} u}{\sigma_{1} \sqrt{2}}, \frac{\bar{m}+\sigma_{2} \sqrt{2} u}{\sigma_{1} \sqrt{2}} ; \nu\right) e^{-u^{2}} d u \\
= & \int_{-\frac{m_{2}}{\sigma_{2} \sqrt{2}}}^{\infty} \int_{\frac{\tilde{x}-\bar{m}-\sigma_{2} \sqrt{2} u}{\sigma_{1} \sqrt{2}}}^{\infty} \frac{1}{\left(\frac{\bar{m}+\sigma_{2} \sqrt{2} u}{\sigma_{1} \sqrt{2}}+z\right)^{\nu}} e^{-z^{2}} e^{-u^{2}} d z d u \\
= & \frac{\left(\sigma_{1} \sqrt{2}\right)^{\nu}}{\bar{m}^{\nu}} \int_{-\frac{m_{2}}{\sigma_{2} \sqrt{2}}}^{\infty} \int_{\frac{\tilde{x}-\bar{m}-\sigma_{2} \sqrt{2} u}{\sigma_{1} \sqrt{2}}}^{\infty} \frac{1}{\left(1+u \frac{\sigma_{2} \sqrt{2}}{\bar{m}}+z \frac{\sigma_{1} \sqrt{2}}{\bar{m}}\right)^{\nu}} e^{-z^{2}} e^{-u^{2}} d z d u \\
= & \frac{1}{2} \frac{\left(\sigma_{1} \sqrt{2}\right)^{\nu}}{\bar{m}^{\nu}} \int_{-\infty}^{\frac{m_{2}}{\sigma_{2}}} \int_{-\infty}^{-\frac{\tilde{x}-\bar{m}-\sigma_{2} u}{\sigma_{1}}} \exp \left(-\nu \ln \left(1-u \frac{\sigma_{2}}{\bar{m}}-z \frac{\sigma_{1}}{\bar{m}}\right)-\frac{z^{2}}{2}-\frac{u^{2}}{2}\right) d z d u \\
\simeq & \frac{1}{2} \frac{\left(\sigma_{1} \sqrt{2}\right)^{\nu}}{\bar{m}^{\nu}} \int_{-\infty}^{\frac{m_{2}}{\sigma_{2}}} \int_{-\infty}^{-\frac{\tilde{x}-\bar{m}-\sigma_{2} u}{\sigma_{1}}} \exp \left(\nu \frac{\sigma_{2}}{\bar{m}} u+\nu \frac{\sigma_{1}}{\bar{m}} z-\frac{z^{2}}{2}-\frac{u^{2}}{2}\right) d z d u \\
= & \frac{1}{2} \frac{\left(\sigma_{1} \sqrt{2}\right)^{\nu}}{\bar{m}^{\nu}} \int_{-\infty}^{\frac{m_{2}}{\sigma_{2}}} \int_{-\infty}^{\frac{\bar{m}-\tilde{x}}{\sigma_{1}}} \exp \left(-\frac{1}{2}\left[u^{2}+\left(v-\frac{\sigma_{2}}{\sigma_{1}} u\right)^{2}-2 \nu \frac{\sigma_{2}}{\bar{m}} u-2 \nu \frac{\sigma_{1}}{\bar{m}}\left(v-\frac{\sigma_{2}}{\sigma_{1}} u\right)\right]\right) d v d u \\
= & \frac{\pi\left(\sigma_{1} \sqrt{2}\right)^{\nu}}{\bar{m}^{\nu}} \exp \left(\frac{\nu^{2} \bar{\sigma}^{2}}{2 \bar{m}^{2}}\right) \int_{-\infty}^{\frac{m_{2}}{\sigma_{2}}} \int_{-\infty}^{\frac{\bar{m}-\tilde{x}}{\sigma_{1}}} \frac{1}{2 \pi} \exp \left(-\frac{1}{2}\left[[u-\bar{u} \quad v-\bar{v}] \Sigma^{-1}\left[\begin{array}{c}
u-\bar{u} \\
v-\bar{v}
\end{array}\right]\right]\right) d v d u
\end{aligned}
$$

where $\bar{u}=\frac{\nu \sigma_{2}}{\bar{m}}, \bar{v}=\frac{\nu \bar{\sigma}^{2}}{\sigma_{1} \bar{m}}$ and $\Sigma^{-1}=\left[\begin{array}{cc}1+\frac{\sigma_{2}^{2}}{\sigma_{1}^{2}} & -\frac{\sigma_{2}}{\sigma_{1}} \\ -\frac{\sigma_{2}}{\sigma_{1}} & 1\end{array}\right]$, and consequently $\Sigma=\left[\begin{array}{cc}1 & \frac{\sigma_{2}}{\sigma_{1}} \\ \frac{\sigma_{2}}{\sigma_{1}} & 1+\frac{\sigma_{2}^{2}}{\sigma_{1}^{2}}\end{array}\right]$.

In particular, $|\Sigma|=1$. Thus, we have shown that, for large positive $\bar{m}$ :

$$
\widetilde{H}\left(m_{1}, m_{2}, \sigma_{1}, \sigma_{2} ; \nu\right) \simeq \frac{\pi\left(\sigma_{1} \sqrt{2}\right)^{\nu}}{\bar{m}^{\nu}} \exp \left(\frac{\nu^{2} \bar{\sigma}^{2}}{2 \bar{m}^{2}}\right) \mathcal{N}\left(\frac{m_{2}}{\sigma_{2}}, \frac{\bar{m}-\tilde{x}}{\sigma_{1}} ;\left[\begin{array}{cc}
\frac{\nu \sigma_{2}}{\bar{m}} & \frac{\nu \bar{\sigma}^{2}}{\sigma_{1} \bar{m}}
\end{array}\right],\left[\begin{array}{cc}
1 & \frac{\sigma_{2}}{\sigma_{1}} \\
\frac{\sigma_{2}}{\sigma_{1}} & 1+\frac{\sigma_{2}^{2}}{\sigma_{1}^{2}}
\end{array}\right]\right)
$$




\section{B.14 Proposition 4.11}

Proof. Suppose $\nu \geq 0$. Then:

$$
\begin{aligned}
& \widetilde{H}\left(m_{1}, m_{2}, \sigma_{1}, \sigma_{2} ; \nu\right)=\int_{-\frac{m_{2}}{\sigma_{2} \sqrt{2}}}^{\infty} \int_{\frac{\tilde{x}-\bar{m}-\sigma_{2} \sqrt{2} u}{\sigma_{1} \sqrt{2}}}^{\infty} \frac{1}{\left(\frac{\bar{m}+\sigma_{2} \sqrt{2} u}{\sigma_{1} \sqrt{2}}+z\right)^{\nu}} e^{-z^{2}} e^{-u^{2}} d z d u \\
& =\int_{-\frac{m_{2}}{\sigma_{2} \sqrt{2}}}^{\infty} \int_{\frac{\tilde{x}}{\sigma_{1} \sqrt{2}}}^{\infty} \frac{1}{v^{\nu}} \exp \left(-\left(v-\frac{\bar{m}+\sigma_{2} \sqrt{2} u}{\sigma_{1} \sqrt{2}}\right)^{2}-u^{2}\right) d v d u \\
& \leq\left(\frac{\sigma_{1} \sqrt{2}}{\tilde{x}}\right)^{\nu} \int_{-\frac{m_{2}}{\sigma_{2} \sqrt{2}}}^{\infty} \int_{\frac{\tilde{x}}{\sigma_{1} \sqrt{2}}}^{\infty} \exp \left(-\left(v-\frac{\bar{m}+\sigma_{2} \sqrt{2} u}{\sigma_{1} \sqrt{2}}\right)^{2}-u^{2}\right) d v d u \\
& =\left(\frac{\sigma_{1} \sqrt{2}}{\tilde{x}}\right)^{\nu} \int_{-\infty}^{\frac{m_{2}}{\sigma_{2}}} \int_{-\infty}^{-\frac{\tilde{x}}{\sigma_{1}}+\frac{\bar{m}}{\sigma_{1}}} \exp \left(-\left(-\frac{y}{\sqrt{2}}+\frac{\sigma_{2} x}{\sigma_{1} \sqrt{2}}\right)^{2}-\frac{x^{2}}{2}\right) d y d x \\
& =\left(\frac{\sigma_{1} \sqrt{2}}{\tilde{x}}\right)^{\nu} \int_{-\infty}^{\frac{m_{2}}{\sigma_{2}}} \int_{-\infty}^{-\frac{\tilde{x}}{\sigma_{1}}+\frac{\bar{m}}{\sigma_{1}}} \exp \left(-\frac{1}{2}\left[\begin{array}{ll}
x & y
\end{array}\right]\left[\begin{array}{cc}
1+\frac{\sigma_{2}^{2}}{\sigma_{1}^{2}} & -\frac{\sigma_{2}}{\sigma_{1}} \\
-\frac{\sigma_{2}}{\sigma_{1}} & 1
\end{array}\right]\left[\begin{array}{l}
x \\
y
\end{array}\right]\right) d y d x \\
& =\pi\left(\frac{\sigma_{1} \sqrt{2}}{\tilde{x}}\right)^{\nu} \mathcal{N}\left(\frac{m_{2}}{\sigma_{2}} ;-\frac{\tilde{x}}{\sigma_{1}}+\frac{\bar{m}}{\sigma_{1}} ;\left[\begin{array}{ll}
0 & 0
\end{array}\right] ;\left[\begin{array}{cc}
1 & \frac{\sigma_{2}}{\sigma_{1}} \\
\frac{\sigma_{2}}{\sigma_{1}} & 1+\frac{\sigma_{2}^{2}}{\sigma_{1}^{2}}
\end{array}\right]\right)
\end{aligned}
$$

\section{B.15 Proposition 4.12}

Proof. First, differentiating equation (4.14) yields $\frac{\partial \widetilde{\mathcal{G}}}{\partial x}(x, y ; \nu)=-\frac{1}{(x+y)^{\nu}} e^{-x^{2}}$ and $\frac{\partial \widetilde{\mathcal{G}}}{\partial y}(x, y ; \nu)=$ $-\nu \widetilde{\mathcal{G}}(x, y ; \nu+1)$. Using these expressions to differentiate equation (4.13):

$$
\begin{aligned}
& \frac{\partial \mathcal{G}}{\partial m}(m, \sigma)=-\frac{M}{\sigma} \Phi_{N}\left(\frac{\tilde{x}-m}{\sigma} ; 0,1\right)+\frac{\gamma}{(\sigma \sqrt{2})^{\nu} \sqrt{\pi}}\left(-\frac{1}{\sigma \sqrt{2}} \frac{\partial \widetilde{\mathcal{G}}}{\partial x}\left(\frac{\tilde{x}-m}{\sigma \sqrt{2}}, \frac{m}{\sigma \sqrt{2}} ; \nu\right)+\right. \\
& \left.\frac{1}{\sigma \sqrt{2}} \frac{\partial \widetilde{\mathcal{G}}}{\partial y}\left(\frac{\tilde{x}-m}{\sigma \sqrt{2}}, \frac{m}{\sigma \sqrt{2}} ; \nu\right)\right)=-M \Phi_{N}(\tilde{x} ; m, \sigma)+\frac{\gamma}{\tilde{x}^{\nu} \sigma \sqrt{2 \pi}} \exp \left(-\frac{(\tilde{x}-m)^{2}}{2 \sigma^{2}}\right)- \\
& \frac{\nu \gamma}{(\sigma \sqrt{2})^{\nu+1} \sqrt{\pi}} \widetilde{\mathcal{G}}\left(\frac{\tilde{x}-m}{\sigma \sqrt{2}}, \frac{m}{\sigma \sqrt{2}} ; \nu+1\right)=-\frac{\nu \gamma}{(\sigma \sqrt{2})^{\nu+1} \sqrt{\pi}} \widetilde{\mathcal{G}}\left(\frac{\tilde{x}-m}{\sigma \sqrt{2}}, \frac{m}{\sigma \sqrt{2}} ; \nu+1\right)
\end{aligned}
$$

because $\frac{\gamma}{\widetilde{x}^{\nu}}=M$. Thus, differentiating equation (4.10) and using equation (4.24):

$$
\begin{aligned}
& \frac{\partial H}{\partial m_{1}}\left(m_{1}, m_{2}, \sigma_{1}, \sigma_{2}\right)=\int_{0}^{\infty} \frac{\partial \mathcal{G}}{\partial m}\left(x+m_{1}, \sigma_{1}\right) \Phi_{N}\left(x ; m_{2}, \sigma_{2}\right) d x=-\frac{\nu \gamma}{\left(\sigma_{1} \sqrt{2}\right)^{\nu+1} \sqrt{\pi}} \times \\
& \int_{0}^{\infty} \widetilde{\mathcal{G}}\left(\frac{\tilde{x}-m_{1}-x}{\sigma_{1} \sqrt{2}}, \frac{m_{1}+x}{\sigma_{1} \sqrt{2}} ; \nu+1\right) \Phi_{N}\left(x ; m_{2}, \sigma_{2}\right) d x=-\frac{\nu \gamma}{\pi\left(\sigma_{1} \sqrt{2}\right)^{\nu+1}} \widetilde{H}\left(m_{1}, m_{2}, \sigma_{1}, \sigma_{2} ; \nu+1\right) \\
& \frac{\partial H}{\partial m_{2}}\left(m_{1}, m_{2}, \sigma_{1}, \sigma_{2}\right)=-\int_{0}^{\infty} \mathcal{G}\left(x+m_{1}, \sigma_{1}\right) \frac{\partial \Phi_{N}}{\partial x}\left(x ; m_{2}, \sigma_{2}\right) d x \\
& =\mathcal{G}\left(m_{1}, \sigma_{1}\right) \Phi_{N}\left(0 ; m_{2}, \sigma_{2}\right)+\int_{0}^{\infty} \frac{\partial \mathcal{G}}{\partial x}\left(x+m_{1}, \sigma_{1}\right) \Phi_{N}\left(x ; m_{2}, \sigma_{2}\right) d x \\
& =\mathcal{G}\left(m_{1}, \sigma_{1}\right) \Phi_{N}\left(0 ; m_{2}, \sigma_{2}\right)-\frac{\nu \gamma}{\pi\left(\sigma_{1} \sqrt{2}\right)^{\nu+1}} \widetilde{H}\left(m_{1}, m_{2}, \sigma_{1}, \sigma_{2} ; \nu+1\right)
\end{aligned}
$$

Now, for $1 \leq i \leq n$ and $1 \leq k \leq n$, we differentiate equations (4.11):

$$
\frac{\partial \bar{m}_{i}^{n}}{\partial d_{t}}=0 \quad, \quad \frac{\partial \bar{m}_{i}^{n}}{\partial C_{t}^{k}}=e^{-\alpha_{k}(T-t)} \mathbf{1}_{\{i \leq k\}} \quad, \quad \frac{\partial \bar{m}_{1}^{i, D}}{\partial d_{t}}=-e^{-\alpha_{D}(T-t)} \quad, \quad \frac{\partial \bar{m}_{1}^{i, D}}{\partial C_{t}^{k}}=e^{-\alpha_{k}(T-t)} \mathbf{1}_{\{k \leq i\}}
$$

Using all these results, the differentiation of equations (4.9) and (4.12) is straightforward. 


\section{Algorithms}

This appendix provides the guidelines to compute efficiently the trickiest quantities from section 4.2. The other quantities are straightforward to compute from these building blocks.

\section{C.1 Extended Incomplete Gamma Function}

Following the algorithm described in Section 4.2.5, we give details of a numerically stable computation of $\Gamma\left(\alpha, x ; b ;-\frac{1}{2}\right)$. We exploit the sum (4.19) and the recurrence relation (4.20). Denote by $x_{n}$ the $n$-th term of the sum :

$$
x_{n}:=\Gamma\left(\alpha+\frac{n}{2}, x\right) \frac{(-b)^{n}}{n !}
$$

Then, for $n \geq 2$, using (4.20) :

$$
x_{n}=\frac{b^{2}}{n(n-1)} a_{n} x_{n-2}+c_{n}
$$

where $a_{n}=a+\frac{n-2}{2}$ and $c_{n}=e^{-x} x^{a+\frac{n-2}{2}} \frac{(-b)^{n}}{n !}$, computed using $a_{n}=a_{n-1}+\frac{1}{2}$ and $c_{n}=-\frac{b}{n} \sqrt{x} c_{n-2}$. Using these relations, the implementation of an algorithm to compute the EIG function is straightforward.

\section{C.2 Extended Incomplete Goodwin-Staton Integral}

Combining the different methods described in Section 4.2.5, the implementation of an algorithm to compute $\widetilde{\mathcal{G}}(x, y ; \nu)$ is also straightforward. The only remaining detail is to choose when to switch from one method to the other. We propose to use the large positive $y$ approximation when $y>10$, to test the large negative $y$ upper bound whenever $y<0$ (before using the series expansion if the upper bound is consider not small enough, say $>10^{-30}$ ), and the series expansion in the remaining cases.

\section{C.3 $\widetilde{H}\left(m_{1}, m_{2}, \sigma_{1}, \sigma_{2} ; \nu\right)$}

The first step is to build an efficient implementation of the sum (4.25). Let us skip the multiplicative constant for the moment. Denote by $x_{n}$ the $n$-th term of the sum :

$$
x_{n}:=\Gamma\left(a+\frac{n}{2}, x\right) \widetilde{\mathcal{G}}(X, Y ;-n) \frac{b^{n}}{n !}
$$

where $x, b, X$ and $Y$ are constants. In order to exploit the recurrence relations (4.15) and (4.20), for efficiency, while being numerically stable, we decompose $x_{n}$ as:

$$
x_{n}=p_{n} q_{n} \quad, \quad p_{n}=\Gamma\left(a+\frac{n}{2}, x\right) \frac{(\sqrt{b})^{n}}{\prod_{i=1}^{n} \sqrt{i}} \quad, \quad q_{n}=\widetilde{\mathcal{G}}(X, Y ;-n) \frac{(\sqrt{b})^{n}}{\prod_{i=1}^{n} \sqrt{i}}
$$

For $n \geq 2$, the recurrence relations result in:

$$
p_{n}=b \frac{a-1+\frac{n}{2}}{\sqrt{n} \sqrt{n-1}} p_{n-2}+c_{n} \quad, \quad q_{n}=\frac{b}{2} \frac{\sqrt{n-1}}{\sqrt{n}} q_{n-2}+Y \frac{\sqrt{b}}{\sqrt{n}} q_{n-1}+d_{n}
$$

where $c_{n}:=x^{a-1} e^{-x} \frac{(\sqrt{b x})^{n}}{\prod_{i=1}^{n} \sqrt{i}}$ and $d_{n}:=\frac{1}{2} \frac{e^{-X^{2}}}{X+Y} \frac{((X+Y) \sqrt{b})^{n}}{\prod_{i=1}^{n} \sqrt{i}}$ are computed using $c_{n}=\frac{\sqrt{b x}}{\sqrt{n}} c_{n-1}$ and $d_{n}=\frac{(X+Y) \sqrt{b}}{\sqrt{n}} d_{n-1}$.

Using these relations, the implementation of an algorithm to compute the sum (4.25) is straightforward. Combining it with the different algorithms from Section $4.2 .6, \widetilde{H}\left(m_{1}, m_{2}, \sigma_{1}, \sigma_{2} ; \nu\right)$ is easily computed. Again it remains to choose when to switch from one method to the other. We propose to use the large positive $y$ approximation when $y>10 \max \left(\sigma_{1}, \sigma_{2}\right)$, to test, if $\nu>0$, the large negative $y$ upper bound whenever $y<0$ (before using the series expansion if the upper bound is consider not small enough, say $\left.>10^{-30}\right)$, and the series expansion in the remaining cases. 